NBER WORKING PAPER SERIES

\title{
A FUNCTIONAL FILTERING AND NEIGHBORHOOD TRUNCATION APPROACH TO INTEGRATED QUARTICITY ESTIMATION
}

\author{
Torben G. Andersen \\ Dobrislav Dobrev \\ Ernst Schaumburg \\ Working Paper 17152 \\ http://www.nber.org/papers/w17152 \\ NATIONAL BUREAU OF ECONOMIC RESEARCH \\ 1050 Massachusetts Avenue \\ Cambridge, MA 02138 \\ June 2011
}

We thank participants at the 2010 SETA Conference at Singapore Management University and the "Nonlinear and Financial Econometrics Conference: A Tribute to A. Ronald Gallant," Toulouse, France, May 2011, along with Federico Bandi, Peter R. Hansen, Andrew Patton, Peter C. B. Phillips and Kevin Sheppard for comments on an earlier draft. Excellent research assistance was provided by Patrick Mason. Andersen gratefully acknowledges financial support from the NSF through a grant to the NBER and by CREATES funded by the Danish National Research Foundation. The views in this paper are solely those of the authors and should not be interpreted as reflecting the views of the Board of Governors of the Federal Reserve System, the Federal Reserve Bank of New York, any other person associated with the Federal Reserve System, or the National Bureau of Economic Research.

NBER working papers are circulated for discussion and comment purposes. They have not been peerreviewed or been subject to the review by the NBER Board of Directors that accompanies official NBER publications.

(C) 2011 by Torben G. Andersen, Dobrislav Dobrev, and Ernst Schaumburg. All rights reserved. Short sections of text, not to exceed two paragraphs, may be quoted without explicit permission provided that full credit, including (C) notice, is given to the source. 
A Functional Filtering and Neighborhood Truncation Approach to Integrated Quarticity Estimation Torben G. Andersen, Dobrislav Dobrev, and Ernst Schaumburg

NBER Working Paper No. 17152

June 2011

JEL No. G12,G13,G17

\begin{abstract}
$\underline{\text { ABSTRACT }}$
We provide a first in-depth look at robust estimation of integrated quarticity (IQ) based on high frequency data. IQ is the key ingredient enabling inference about volatility and the presence of jumps in financial time series and is thus of considerable interest in applications. We document the significant empirical challenges for IQ estimation posed by commonly encountered data imperfections and set forth three complementary approaches for improving IQ based inference. First, we show that many common deviations from the jump diffusive null can be dealt with by a novel filtering scheme that generalizes truncation of individual returns to truncation of arbitrary functionals on return blocks. Second, we propose a new family of efficient robust neighborhood truncation (RNT) estimators for integrated power variation based on order statistics of a set of unbiased local power variation estimators on a block of returns. Third, we find that ratio-based inference, originally proposed by Barndorff-Nielsen and Shephard, has desirable robustness properties and is well suited for our empirical applications. We confirm that the proposed filtering scheme and the RNT estimators perform well in our extensive simulation designs and in an application to the individual Dow Jones 30 stocks.
\end{abstract}

Torben G. Andersen

Kellogg School of Management

Northwestern University

2001 Sheridan Road

Evanston, IL 60208

and NBER

t-andersen@kellogg.northwestern.edu

Dobrislav Dobrev

Federal Reserve Board of Governors

20th Street and Constitution Avenue NW

Washington, DC 20551

Dobrislav.P.Dobrev@frb.gov
Ernst Schaumburg

Federal Reserve Bank of New York

33 Liberty Street

New York, NY 10045

ernst.schaumburg@ny.frb.org 


\section{Introduction}

It is by now widely accepted that intraday data are highly informative regarding the contemporaneous level of volatility relative to daily returns. The use of high-frequency returns is, however, not without its problems. The main issue is the pronounced inhomogeneity of the intraday return series across the trading day as diurnal patterns interspersed with news events and market microstructure frictions complicate direct modeling of the high-frequency volatilities and introduces a variety of idiosyncratic features that are largely irrelevant for longer horizon inter-daily volatility. The realized volatility (RV) approach "solves" this problem by aggregating the intraday return observations to a daily frequency in a manner that retains the majority of the inherent volatility information while removing the bulk of any irregular noise or diurnal patterns. The RV approach has been widely adopted in applications involving liquid asset markets and availability of intraday price, ever since the formal introduction of RV as a nonparametric consistent estimator of the return variation for arbitrage-free return processes in Andersen and Bollerslev (1998). ${ }^{1}$ In parallel with the rapidly growing number of empirical RV applications a large body of theoretical work on model-free, nonparametric estimation and inference for components of the realized return variation process of continuous-time semimartingales has arisen. Initial econometric issues are addressed in Andersen, Bollerslev, Diebold and Labys (2001, 2003) and Barndorff-Nielsen and Shephard (henceforth BNS) (2002), while additional work on inference regarding the contribution of jumps and the precision of the integrated continuous return variation is developed in BNS $(2002,2004 b, 2006){ }^{2}$ Subsequently, this literature further developed into dealing with market microstructure issues as the drive towards exploiting data at the utmost highest frequencies was pursued by, e.g., Zhang, Mykland and Ait-Sahalia (2005), Bandi and Russell (2008), and Barndorff-Nielsen, Hansen, Lunde and Shephard, henceforth BNHLS, (2008).

The main conceptual distinction between realized volatility and standard volatility modeling is that the former focuses on ex-post measurement of the actual realization of the (stochastic) return variation process over a given period while the latter produces ex-ante expectations of the future return variance. Once the attention shifts towards actual realizations, it is natural to push the framework further and seek additional information from the high-frequency returns. In particular, the full potential of the RV approach is not attained until we can provide robust and reliable procedures for ex-post day-by-day measures of the precision of the integrated return variation and identify the impact of jump components. Both features are critical for a variety of issues in real-time financial management, including improved volatility forecasting, analysis of the dynamic properties of jumps and

\footnotetext{
${ }^{1}$ Corresponding measures were previously considered informally on an ad hoc basis, see, e.g., Schwert (1989), Hsieh (1991) and Zhou (1996).

${ }^{2}$ Related general technical insights are provided in Jacod and Protter (1998).
} 
news events, derivatives pricing, estimation of return correlations, determination of returnvolatility asymmetries (the leverage effect), and for developing insights into asset pricing via better understanding of the empirical interplay between the return volatility and covariation and the general macroeconomic conditions.

The critical ingredient for inference regarding the return variation and for the reliability of jump test procedures is the so-called integrated quarticity (IQ). To illustrate the importance of accurate IQ measures, we informally review a few basic results from the RV literature. Let the continuously evolving logarithmic (log-) price for a single asset be given by $Y_{t}$. This price process constitutes a semimartingale with respect to an underlying filtered probability space. The associated ex-post daily realized quadratic variation $(Q V)$ for $Y_{t}$ may be decomposed into an integrated (diffusive) volatility, $I V$, and a residual (jump) component, $J V$ :

$$
\begin{aligned}
d Y_{t} & =\mu_{t} d t+\sigma_{t} d W_{t}+d J_{Y, t} \\
Q V_{t} & =\underbrace{\int_{0}^{t} \sigma_{u}^{2} d u}_{I V}+\underbrace{\sum_{0 \leq u \leq t}\left(\Delta J_{Y, u}\right)^{2}}_{J V}
\end{aligned}
$$

where $\mu_{t}$ and $\sigma_{t}$ denote the instantaneous drift and diffusion coefficients, while $W_{t}$ and $J_{Y, t}$ are adapted Wiener and finite activity jump processes respectively.

For a given trading day covering $t \in[0,1]$, we consider the ideal scenario in which we observe $N$ equally-spaced $(\log )$ returns, $\quad r_{i}=Y_{\frac{i}{N}}-Y_{\frac{i-1}{N}}, \quad i=1, \cdots, N . \quad$ In this case, the realized volatility $(\mathrm{RV})$ is a consistent nonparametric estimator of $\mathrm{QV}$, as the number of intraday observations diverges, $N \rightarrow \infty$ (in-fill asymptotics):

$$
R V_{N}=\sum_{i=1}^{N} r_{i}^{2} \rightarrow Q V .
$$

Furthermore, in the absence of price jumps, the limiting distribution of the RV estimator is known to be a Gaussian mixture of the following form,

$$
\sqrt{N}\left(R V_{N}-I V\right) \rightarrow \mathcal{N}(0,2 I Q)
$$

where $I Q=\int_{0}^{1} \sigma_{u}^{4} d u$ which, as observed by BNS (2002), can be consistently estimated from the high-frequency data themselves via the Realized Quarticity (RQ) statistic:

$$
R Q_{N}=\frac{N}{3} \sum_{i=1}^{N} r_{i}^{4} \rightarrow I Q
$$

It is evident that reliable estimates for IQ are critical for accurate inference about the integrated variance. Unfortunately, IQ estimation is challenging because it involves estimating fourth order return moments from a large set of noisy intraday returns characterized 
by numerous outliers and the confounding effects of market microstructure frictions. For example, it is well recognized that the RQ estimator above is quite imprecise and highly non-robust in many realistic scenarios, even if jumps are absent. Moreover, the problem only grows worse in the presence of jumps as the standard nonparametric RV theory is highly non-robust in this dimension. First, when price jumps are present, RV is no longer consistent for IV. Second, the RQ statistic is now divergent: $R Q_{N} \rightarrow \infty$ as $N \rightarrow \infty$. Given the compelling evidence for jumps in many prominent asset price series, further corroborated below, this is critical in practice. Consequently, one may conclude that inference on the integrated variance or the presence of jumps should, as a matter of routine, exploit jump-robust measures for integrated quarticity (and integrated variance). While various such jump-robust estimators have been developed, they are known to be rather imprecise, at least in some empirically relevant settings, and there simply exists no systematic evidence regarding the performance of alternative jump-robust procedures across a range of realistic empirical scenarios.

Recognizing the above problems, a variety of different, and largely ad hoc, procedures for IQ estimation have been proposed and implemented in the empirical literature. The early literature often maintained the assumption of a purely diffusive price process when assessing the precision of the integrated variance, enabling direct reliance on the basic RV theory. For example, BNS (2004a) exploit 10-minute foreign exchange returns in estimating IQ through a statistic closely related to RQ. Similarly, Bandi and Russell (2008) recommend using RQ sampled at the 15-20 minute frequency for estimating IQ. These relatively conservative sampling intervals reflect the sensitivity of fourth moment returns to market microstructure noise and outliers: these effects are attenuated as the return frequency shrinks. On the other hand, robust IQ estimators are essential for statistical power in jump tests so jumprobust procedures invariably have been used in that context. For example, BNS (2004b) rely on 5-minute returns for constructing the so-called quadpower estimator of IQ while Huang and Tauchen (2005) also exploit 5-minute returns for computing a variety of jumprobust IQ measures, including the tripower and quadpower statistics. ${ }^{3}$ Both sets of authors provide evidence regarding the reliability of the procedures, but the focus is strictly on the performance of the jump test statistic and not on the IQ estimator. In fact, recognizing the considerable imprecision of such jump-robust IQ estimators, Jiang and Oomen (2008) opt for simply squaring their jump-robust IV estimator, thus settling for a Jensen inequality bias in their IQ estimator rather than relying on prevailing consistent, but arguably more inaccurate, jump-robust estimators. In particular, Jiang and Oomen (2008) worry about distortions due to market microstructure noise when applying standard jump-robust IQ estimators to their one-minute IBM transaction return series.

As an illustration of the practical importance of jump robustness, consider drawing

\footnotetext{
${ }^{3}$ We formally define these estimators in the next section.
} 
inference about the IV of IBM stock returns across three days in February 2008 using the non-jump robust RQ/RV measures versus a pair of jump robust measures ${ }^{4}$ :
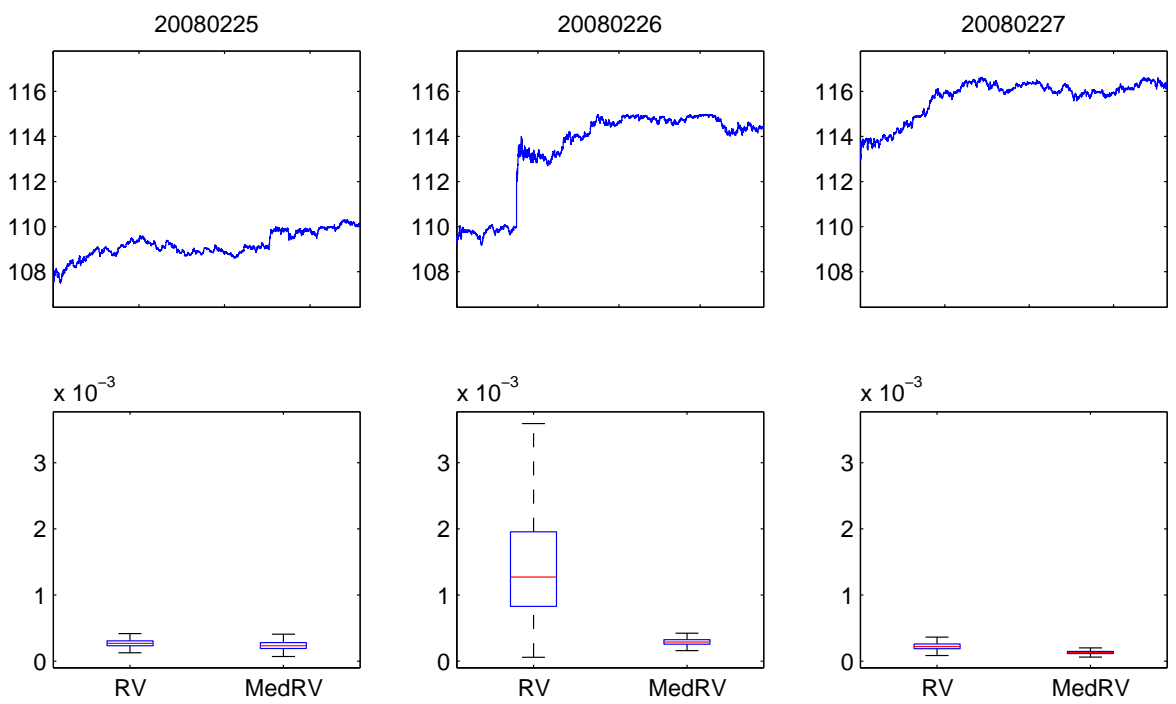

Figure 1: The point estimate (red line), and the inter-quartile range as well as two standard deviation IV confidence bands for IBM for three trading days in February 2008.

The jump on $2 / 26 / 2008$ is readily identified visually and easily detected using a jump robust test statistic. In fact, the robust MedRV estimates and associated standard error bands, based on MedRQ, suggest a relative stable volatility process across the three trading days. In contrast, the regular RV estimate for IV is greatly inflated on $2 / 26 / 2008$, and the confidence band is huge, reflecting a diverging RQ statistic. Hence, the reliance on non jump-robust statistics has two consequences. First, when jumps are present the IV estimate is upward biased because the jump component in QV is attributed to IV. ${ }^{5}$ Second, the associated confidence band is grossly overstated, indicating very poor estimation precision whereas, in fact, the robust estimate appears quite reliable. Hence, non-robust inference may produce excessively erratic IV estimates and convey a sense of exaggerated imprecision associated with these techniques. While the misleading inference afforded by the regular RV and RQ estimators is apparent in Figure 1, at least when contrasted with the robust inference and a depiction of the price path, it can be less obvious in cases with higher volatility levels and relatively smaller jumps. As such, it is important to develop feasible

\footnotetext{
${ }^{4}$ We rely on the MedRV/MedRQ estimators of Andersen, Dobrev and Schaumburg (2009) here, but any other sensible pair of robust IV and IQ estimators would suffice in this particular case as the evidence for a single significant jump is compelling and the associated empirical inference problem thus very straightforward. The robust estimators will be introduced later in the paper.

${ }^{5}$ As a consequence, real-time predictions regarding the expected volatility over the following trading days will likely also be exaggerated on $2 / 26 / 2008$ because the jump component of QV typically is much less persistent than the IV component, see, e.g., Andersen, Bollerslev and Diebold (2007).
} 
robust and efficient procedures for estimating IQ and conducting inference for IV.

One main contribution of this paper is to explore the virtues and drawbacks of alternative estimation procedures for IQ, including their robustness and sensitivity to a variety of features of the return generating process and the presence of relevant market frictions. Although a variety of authors in recent years have extended their estimators to also cover higher order integrated power variation, the present paper, to the best of our knowledge, constitutes the first in-depth look at the performance of robust estimation procedures for IQ based on high-frequency financial data when the underlying price process contains jumps. A second contribution is the development of an outlier filtering procedure that operates directly on an estimation functional of interest rather than on the individual tick-by-tick returns. This functional filtering principle serves to adapt the filter to the specific assumptions underlying a given estimator. Hence, the filter controls the impact, and potential distortion, of abnormal outliers in the exact metric in which they contribute to the ultimate estimator. By design, this filter can be applied extremely conservatively under the null hypothesis so that it has a negligible impact on the properties of the estimators. In applications to individual equity return data we find this filter indispensable for rendering entire classes of promising candidate IQ estimators viable. Third, we develop a new class of robust neighborhood truncation (RNT) estimators that generalize existing nearest neighbor and quantile RV estimators. They involve the application of a second layer of order statistics to suitably chosen return functionals, thus robustifying the inference for IQ without sacrificing much efficiency. We find such RNT estimators to perform admirably, especially when exploited in combination with the ratio statistic, $I Q / I V^{2}$, which previously has been found to provide improved finite sample inference for IV and enhance the power of jump tests. The unifying theme behind our new class of estimators and universal filtering procedure is to operate directly on the functional space of local power variation estimates rather than the underlying individual returns, which constitutes a special case.

The remainder of the paper is structured as follows. In section 2 we briefly review the principles behind the modern approach to robust estimation of integrated power variation, including IV and IQ, and introduce our novel filtering procedure. Section 3 reviews existing estimators and associated techniques for controlling the impact of outliers and develops our new class of robust neighborhood truncation estimators for IV and IQ. In section 4 we conduct an extensive simulation study to elucidate the potential importance of common types of data features as well as imperfections and how these traits impact alternative IQ estimators. Finally, in Section 5, we analyze the set of Dow Jones 30 stocks between January 2005 and July 2009. We highlight the importance of (mild) filtering and study the relative performance of a wide range of robust IQ estimators. Section 6 concludes. 


\section{Jump-Robust Power Variation Estimation and Filtering}

We begin with an informal review of the modern approach to integrated power variation estimation. In the process, we discuss some important trade-offs that must be confronted in empirical work when dealing with objects involving high powers of volatility, such as integrated quarticity. In particular, we argue that existing filters, developed for realized volatility measures, are inadequate to deal with the complications associated with many relevant jump-robust procedures that exploit overlapping blocks of adjacent returns. Hence, we end by highlighting a novel filtering procedure which is instrumental for obtaining highquality empirical measures of higher order integrated power variation for a promising set of novel robust estimators that we introduce later on.

\subsection{The Modern Approach to Jump-Robust Volatility Estimation}

The basic estimation strategy is straightforward. First, no-arbitrage conditions in a frictionless continuous-time setting ensures that the log-price process is a semimartingale. Hence, over short time intervals, the expected returns are an order of magnitude smaller than the realized return innovations, so the drift term may typically be ignored in deriving the asymptotic distribution for volatility estimators. Second, given sufficient regularity, we may break the trading period, say, $[0,1]$ into $K$ smaller blocks and treat volatility as constant within each block, even if the return variation evolves stochastically and the price path contains finite activity jumps. Combined, these features greatly simplify matters, as nonparametric jump-robust estimators are easy to devise when volatility is constant. The strategy is then simply to estimate the local (power of) volatility for each block and cumulate across blocks to obtain the overall integrated power variation.

\subsubsection{Consistent Estimation under the Diffusive Null Hypothesis}

Assume we have $m+1$ equally-spaced log-price observations, and thus $m$ continuously compounded returns, per block. The total number of returns per trading period is then $N=K \cdot m$. Each block covers $\frac{1}{K}^{\text {th }}$ of the period and each return reflects the price evolution over an interval length of $\frac{1}{N}$. The associated distribution theory is developed using standard in-fill asymptotics, letting $N$ grow indefinitely, while requiring $\frac{m}{K} \rightarrow 0$. In most cases, $m$ is fixed and $K$ diverges directly in line with $N$.

A generic estimator of the $p^{\text {th }}$ order return variation, for $p$ an even positive integer, is obtained as a sum of local estimates of $\sigma^{p}$ based on a functional $f$ operating on blocks of $m$ adjacent (absolute) returns. ${ }^{6}$ The specification of the functional depends on the estimator

\footnotetext{
${ }^{6}$ In general, these returns may themselves have been generated via standard noise-reduction techniques such as sub-sampling or pre-averaging, possibly performed on prices that have been pre-filtered for errors in the raw tick-by-tick data. We address the need for filtering procedures in this context below.
} 
adopted, which is not critical for the current exposition. ${ }^{7}$ For each integer $i, 1 \leq i \leq N-$ $m+1$, we have the return block, $\left(r_{i}, r_{i+1}, \ldots, r_{i+m-1}\right)$. Under the null hypothesis that the returns within the block are distributed i.i.d. $N\left(0, \sigma_{i}^{2} / N\right)$, we let $f_{i}=f\left(\left|r_{i}\right|, \ldots,\left|r_{i+m-1}\right|\right)$ denote the functional exploited by a specific estimator to obtain an unbiased estimate of $\sigma_{i}^{p} / N$ for the $i$ 'th block. The associated estimator of the integrated $p^{\text {th }}$ order variation is,

$$
\frac{N}{N-m+1} \sum_{i=1}^{N-m+1} f_{i}
$$

The consistency of the estimator follows as, subject to sufficient regularity, we have, ${ }^{8}$

$$
\frac{1}{N-m+1} \sum_{i=1}^{N-m+1} N \cdot f_{i} \approx \frac{1}{N-m+1} \sum_{i=1}^{N-m+1} \sigma_{i}^{p} \rightarrow \int_{0}^{1} \sigma_{u}^{p} d u .
$$

A few critical issues determine whether the approach works well in practice. First, the ideal assumptions ignore the impact of data errors and market microstructure frictions. Higher order return moments can be particularly sensitive to faulty price observations or inappropriate assumptions regarding the evolution of ultra-high frequency return data. Second, even if jump robust estimators are designed to avoid, or greatly alleviate, any asymptotic bias stemming from discontinuities, jumps will nonetheless often have a severe adverse effect on the finite sample properties of the estimators, especially for $p>2$. The following sections discuss the basic strategy for dealing with such issues.

\subsubsection{Eliminating Obvious Errors from the Tick-by-Tick Price Series}

Any large set of raw transactions data is invariably subject to recording errors that infuse noise into the observed high-frequency returns. Most dramatically, faulty prices create artificial outliers, causing so-called "bounce-backs" in returns, as there is a "jump" both when the flawed price first appears and then later, often shortly thereafter, when the price reverts to the correct level. Hence, the need for effective cleaning procedures has long been acknowledged. BNHLS (2009) lay out a systematic framework for dealing with trade data from NYSE-TAQ. In their terminology, we apply the filters P1-P3 and T1-T4. ${ }^{9}$ These filters are arguably mild and uncontroversial and simply aim to eliminate obvious data errors.

\footnotetext{
${ }^{7}$ Candidates include the multipower variation, truncated power variation, and quantile power variation estimators.

${ }^{8}$ Of course, this is purely heuristic. The asymptotic properties of the relevant estimators must be proven rigorously in each case.

${ }^{9} \mathrm{P} 1$ : Retain only observations with time stamps between 9:30am and 4:00pm. P2: Retain only trades with positive prices. P3: Retain only trades originating from the main exchange (NYSE for all stocks except MSFT and INTL for which it is NASDAQ). T1: Delete entries with corrected trades. T2: Delete entries with abnormal sale condition. T3: If multiple trades occurred with the same time stamp, use the median price. T4: Delete entries with prices above the ask (or under the bid) by more than one bid-ask spread.
} 
In estimating higher order return power variation measures, we deal with procedures that are highly sensitive to erroneous outliers. As such, the existing conservative error filtering techniques, including those discussed above, are insufficient. In a separate subsection below, we detail our construction of a suitable filter to deal with the robust power variation estimators operating on return blocks.

\subsubsection{Alleviating the Impact of Microstructure Features}

In practice, the theoretical assumptions justifying the presence of a diffusive return component are systematically violated at the tick-by-tick level by various market microstructure features, including the finite price grid and the presence of a bid-ask spread. This implies that tick-by-tick price changes often are an order of magnitude larger than what is consistent with a diffusive characterization. One effective approach to mitigating the impact of such noise is to apply so-called pre-averaging, as originally suggested by Podolskij and Vetter (2009). This is achieved by collapsing a higher number of ultra high-frequency returns into a smaller set of averaged, and thus less erratic "smoothed," returns. This implies that each of the $m$ returns within a block are obtained as averages from separate, non-overlapping subsets of tick-by-tick returns. The benefit is a dramatic reduction in the impact of idiosyncratic noise and, in particular, the distortions introduced by bounce-backs. The drawback is a substantial drop in the underlying sampling frequency. The latter impacts the choice of the window width, $m$, as the (diffusive) volatility fluctuates more widely across longer blocks. We apply the pre-averaging procedure in the simulation and empirical sections below. Our implementation of the pre-averaging technique is detailed in Appendix C.

\subsubsection{Jump-Robust Power Variation Estimation}

Finally, we outline the basic principles behind the construction of return variation estimators that are robust to the presence of finite activity jump processes. Asymptotically, as the block sizes shrink towards zero and the number of blocks grows indefinitely, there will be a finite number of blocks containing a single jump each. Hence, in the limit, the power variation associated with the blocks containing jumps is negligible. It follows that the power variation can be estimated consistently as long as the contribution from the "jump blocks"

is an order of magnitude less than the overall power variation measure, $\int_{0}^{1} \sigma_{u}^{p} d u$, which of course is $O(1)$. However, the jumps are themselves of order $O(1)$ so the functional $f$ must ensure that the jumps are dampened sufficiently to eliminate their impact asymptotically.

More formally, for any given sampling frequency, we may denote the set of indices corresponding to returns for which the associated block contains a jump by $I_{J}$. Thus, for $i \in I_{J}$, there is a jump within the return block $\left(r_{i}, r_{i+1}, \ldots, r_{i+m-1}\right)$. We may then write 
the generic power variation estimator as,

$$
\frac{N}{N-m+1} \sum_{i=1}^{N-m+1} f_{i}=\frac{N}{N-m+1}\left(\sum_{i=1, i \notin I_{J}}^{N-m+1} f_{i}+\sum_{i \in I_{J}} f_{i}\right) .
$$

The first term estimates the integrated power variation consistently, i.e.,

$$
\frac{N}{N-m+1} \sum_{i=1, i \notin I_{J}}^{N-m+1} f_{i} \longrightarrow \int_{0}^{1} \sigma_{u}^{p} d u, \quad \text { as } N \longrightarrow \infty .
$$

The contribution from the blocks containing jumps is negligible, in the limit, if each such block is of order less than $O(1)$. Thus, the associated power variation estimator is consistent as long as $f_{i} \sim O\left(N^{-h}\right), h>0$, for $i \in I_{J} .{ }^{10}$ This is accomplished in different ways by the estimators reviewed in Sections 3.1-3.3. Moreover, their practical effectiveness is largely determined by the degree to which they accomplish sufficient dampening of the jump contributions in finite samples.

\section{$2.2 \quad$ Filtering via Truncation of Return Functionals}

Even for returns based on pre-averaged tick data and sampled at moderate frequencies, microstructure features and other data irregularities may induce inhomogeneous and serially correlated observations that blatantly violate our distributional assumptions. For quarticity estimation, in particular, it is paramount to control the impact of this type of data imperfections to achieve a beneficial trade-off between robustness and efficiency.

This section outlines a general truncation principle for return functionals that enhances the robustness of integrated power variation estimators operating on return blocks. It may be viewed as an extension of existing techniques that employ truncation to alleviate the impact of jumps or data errors. However, the philosophy and implementation are very different. Existing procedures truncate returns based on whether a single observation constitutes a significant outlier. Moreover, the truncation is an essential step in rendering the estimator robust as it dampens, and asymptotically eliminates, the distortion induced by price jumps on the estimated power variation. For this to be effective, the detection of larger jumps must be reliable, and it is common to apply a threshold for jumps that correspond to "three sigmas" or a p-value of about $0.3 \%$. As a result, the procedure generates a nontrivial amount of type I errors because diffusive returns based on high-frequency return data inevitably will be subject to unwarranted truncation. Recognizing the resulting downward bias under the null hypothesis, Corsi, Pirino and Reno (2010), henceforth CPR, develop a rescaling procedure intended to alleviate this effect.

\footnotetext{
${ }^{10}$ Naturally, additional dampening is required to establish a limiting distribution theory (Central Limit Theory or CLT) for the power variation measures. In this case, $f_{i} \sim O\left(N^{-h}\right), h>\frac{1}{2}$, for $i \in I_{J}$.
} 
In contrast, we develop a filtering procedure that operates directly on the jump-robust functional and is intended more broadly to alleviate distortions induced by deviations from the null that the block consists of i.i.d. draws from a normal distribution. In this scenario, jump robustness is, in principle, already assured by the choice of an appropriate functional. Hence, the filtering is merely intended to eliminate truly excessive estimates of local power variation, driven by functional values incompatible with the maintained null hypothesis. As such, we rely on an extremely conservative threshold for truncation, typically with p-values around $10^{-6}$ or below. Under the maintained null hypothesis, this implies that we expect to truncate less than one single realization of the return functional across our entire sample. In practice, the underlying assumptions are violated and truncation occurs with non-trivial frequency which helps control the associated distortion in the power variation estimators. The flowchart shown in Figure 2 illustrates the difference between the truncation of individual returns and the truncation of local estimates, which themselves are functionals of blocks of adjacent returns.
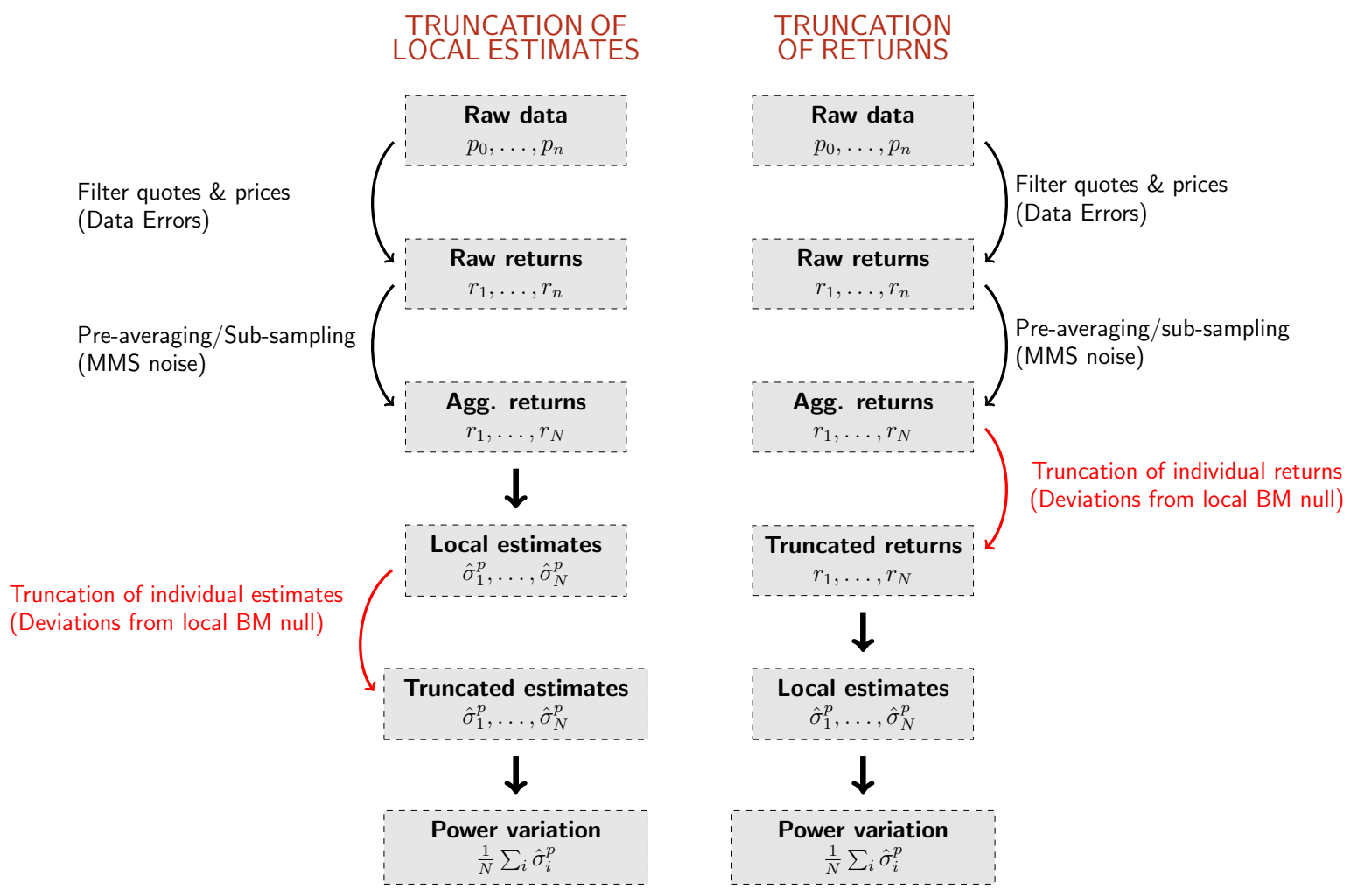

Figure 2: Flowchart contrasting the standard filtering approach based on truncation of individual returns with the proposed new filtering approach based on truncation of individual local estimates. 


\subsubsection{The Infeasible Functional Truncation Filter}

We first provide an infeasible version of the filtering procedure. Recall that we define $N \cdot f_{i}=N \cdot f\left(\left|r_{i}\right|, \ldots,\left|r_{i+m-1}\right|\right)$ as the functional providing an unbiased estimator of the local power variation, $\sigma_{i}^{p}$ under the null hypothesis. Now, for a given $\alpha \in\left(0,10^{-5}\right)$, we let $Q^{(1-\alpha)}[g]$ denote the $(1-\alpha)^{\text {th }}$-quantile of the distribution of a random variable $g$. Set $g_{i}=f_{i} \cdot \sigma_{i}^{-p}$ and define a corresponding truncated functional $f_{i}^{(1-\alpha)}$ as follows:

$$
f_{i}^{(1-\alpha)}= \begin{cases}f_{i} & \text { if } f_{i} \sigma_{i}^{-p} \leq Q^{(1-\alpha)}\left[f_{i} \cdot \sigma_{i}^{-p}\right] \\ 0 & \text { else. }\end{cases}
$$

Accordingly, define the following realized truncated estimator based on $f^{(1-\alpha)}$ :

$$
\frac{N}{\sum_{i=1}^{N-m+1} \boldsymbol{I}\left\{f_{i} \cdot \sigma^{-p}<Q^{(1-\alpha)}\left[f_{i} \cdot \sigma^{-p}\right]\right\}} \sum_{i=1}^{N-m+1} f_{i}^{(1-\alpha)},
$$

where $\boldsymbol{I}\{\boldsymbol{A}\}$ equals one if the expression $\boldsymbol{A}$ is true and zero otherwise.

Setting $\alpha=0$ we obtain the usual realized estimator based on $f$ without truncation. Moreover, if $m=1$ our functional filtering is equivalent to the usual return filtering at the given significance level, $\alpha$.

\subsubsection{The Feasible Functional Truncation Filter}

The truncated estimator defined above requires knowledge of the latent $\sigma_{i}^{p}$ on each block of returns. To obtain a feasible version, we rely on a highly jump-robust and sufficiently local estimator $\hat{\sigma}_{i}^{p}$ calculated on a centered block of returns of size $2 L+1$, where $M<2 L+1<N$. In particular, we employ the following asymptotically unbiased estimators: ${ }^{11}$

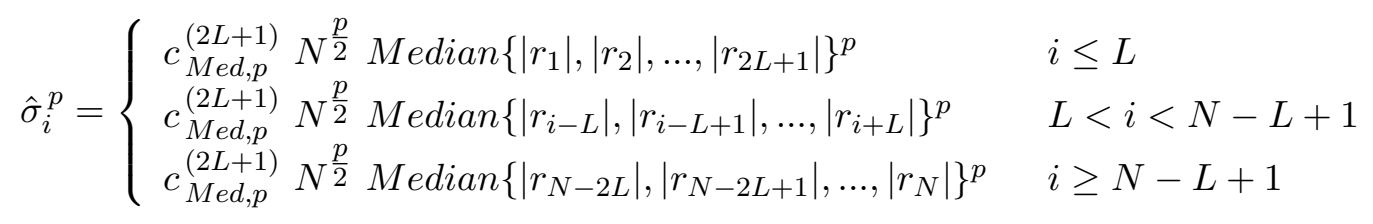

The scaling factor $c_{\text {Med,p }}^{(2 L+1)}$ can be determined with arbitrary precision by simulation. For example, using $L=25$, we obtain $\quad c_{M e d, 2}^{(51)}=2.12$ and $\quad c_{\text {Med,4 }}^{(51)}=4.06$.

The key difference is that the feasible version accommodates the uncertainty arising from using an estimate, $\hat{\sigma}^{p}$, rather than the true $\sigma^{p}$. In particular, we set $\tilde{g}_{i}=f_{i} \cdot\left(\hat{\sigma}_{i}^{p}\right)^{-1}$

\footnotetext{
${ }^{11}$ The efficiency of the estimator is not critical as long as it is sufficiently robust. A Quantile RV type estimator, exploiting a moderate quantile, known to be extremely outlier robust but also downward biased and inefficient, may work well.
} 
and define the truncated functional $\tilde{f}_{i}^{(1-\alpha)}=\tilde{f}^{(1-\alpha)}\left(\left|r_{i}\right|,\left|r_{i+1}\right|, \ldots,\left|r_{i+m-1}\right|\right)$ as follows,

$$
\tilde{f}_{i}^{(1-\alpha)}= \begin{cases}f_{i} & \text { if } f_{i} \cdot\left(\hat{\sigma}_{i}^{p}\right)^{-1} \leq Q^{(1-\alpha)}\left[f_{i} \cdot\left(\hat{\sigma}_{i}^{p}\right)^{-1}\right] \\ 0 & \text { else }\end{cases}
$$

This leads to the following feasible realized truncated estimator based on $\tilde{f}^{(1-\alpha)}$,

$$
\frac{N}{\sum_{i=1}^{N-m+1} \boldsymbol{I}\left\{f_{i} \cdot\left(\hat{\sigma}_{i}^{p}\right)^{-1} \leq Q^{(1-\alpha)}\left[f_{i} \cdot\left(\hat{\sigma}_{i}^{p}\right)^{-1}\right]\right\}} \sum_{i=1}^{N-m+1} \tilde{f}_{i}^{(1-\alpha)}
$$

In summary, our filtering approach has some major advantages. First, by truncating directly the values of the functional $f$ we guard against departures from the null for the return block as a whole. This ensures robustness to consecutive jumps and multiple bouncebacks, exactly for the same reason this is achieved by existing truncation estimators. Second, as noted, it is sufficient to work with very small truncation probabilities (under the null), e.g., $10^{-6}$ or $10^{-7}$, so that for realistic sample sizes one avoids the need for computationallyintensive (recursive) bias correction as implemented by CPR. Third, and most important, the direct truncation of the functional $f$ also limits the potential distortions stemming from a diverse set of other microstructure effects such as basic bid-ask bouncing and price discreteness, but also more challenging effects associated with gradual and directional price changes that may arise from the price dynamics generated by the splitting of large orders or block trades and their interaction with active trading strategies and the reactive behavior of market makers. All such features can potentially induce significant short term serial correlation and non-homogeneity in high frequency returns.

\section{Theory: New and Existing Power Variation Estimators}

\subsection{The Setting and Basic Assumptions}

The main part of our theoretical exposition assumes we operate within a frictionless market where a single financial asset is traded continuously. We deviate from this framework only when we consider the impact of market microstructure noise on our estimation and inference procedures. In this ideal environment, equilibrium ensures that arbitrage opportunities are absent. This implies that the (log) asset price process constitutes a semimartingale with respect to the underlying filtered probability space, see, e.g., Back (1991) and Andersen, Bollerslev and Diebold (2010) for discussion. Hence, for most of the subsequent analysis we invoke the following condition. 
Assumption 1 The continuously compounded return process, $r_{t}$, is governed by a jumpdiffusive semimartingale,

$$
r_{t}=Y_{0}+\int_{0}^{t} a_{u} d u+\int_{0}^{t} \sigma_{u_{-}} d B_{u}+J_{t}
$$

where $a$ is a locally bounded and predictable process, $\sigma$ is an adapted cadlag process bounded away from zero, and $J$ is a finite activity jump process.

When discussing central limit theorems (CLTs) we typically require some additional structure on the volatility process.

Assumption 1A The volatility process, $\sigma_{t}$, follows a jump-diffusive semimartingale,

$$
\sigma_{t}=\sigma_{0}+\int_{0}^{t} \tilde{a}_{u} d u+\int_{0}^{t} \tilde{\sigma}_{u_{-}} d B_{u}+\int_{0}^{t} \tilde{v}_{u_{-}} d W_{u}+J_{\sigma, t}
$$

where $\tilde{a}$ is locally bounded and predictable, $\tilde{\sigma}, \tilde{v}$ are cadlag, the Brownian motions $B, W$ are uncorrelated, and $J_{\sigma}$ is a finite activity jump process.

\subsection{Some Existing Jump-Robust Power Variation Estimators}

\subsubsection{Multi-Power Variation Estimators}

The first (finite activity) jump-robust integrated volatility estimators were the Realized Multi-Power Variation (MPV) statistics, inspired by BNS (2002). Absent frictions, and letting $m>p / 2$, MPV is consistent for the integrated power variation,

$$
\begin{aligned}
& M P V_{N}(m, p)=d_{m, p} \frac{N^{\frac{p}{2}}}{N-m+1} \sum_{i=1}^{N-m+1}\left|r_{i}\right|^{\frac{p}{m}} \cdots\left|r_{i+m-1}\right|^{\frac{p}{m}} \stackrel{P}{\rightarrow} \int_{0}^{1} \sigma_{u}^{p} d u \\
& \text { where } \quad d_{m, p}=\mu_{p / m}^{-m} ; \quad \mu_{p}=E|Z|^{p}=2^{p / 2} \frac{\Gamma((p+1) / 2)}{\Gamma(1 / 2)} ; \quad Z \sim \mathcal{N}(0,1)
\end{aligned}
$$

The intuition for the consistency result is straightforward: If $r_{i}, \ldots, r_{i+m-1} \sim$ i.i.d. $\mathcal{N}\left(0, \sigma^{2}\right)$ then $E\left[\left|r_{i}\right|^{p / m} \cdots\left|r_{i+m-1}\right|^{p / m}\right] \propto \sigma^{p}$ and, suitably normalized, each term of the MPV measure delivers an unbiased estimate of the local (spot) power variation. On the other hand, if there is a single jump within a given block (asymptotically there are never more) then the contribution of this term is dampened by the multiplication of powers of $m-1$ adjacent diffusive returns. It is readily demonstrated that the contribution of the "jump block" is of order $O\left(N^{-1+\frac{p}{2 m}}\right)$. Asymptotically, give the $m>p / 2$ condition, this term is negligible. However, it is also evident that the finite sample distortions of a jump, for fixed $N$, is 
alleviated (declines) as the block length, $m$, increases.

The MPV statistics afford inference for both IV $(p=2)$ and IQ $(p=4)$ as special cases, ${ }^{12}$

$$
\begin{array}{lll}
\sqrt{N}\left[M P V_{N}(m, 2)-I V\right] & \stackrel{\text { Stable } \mathcal{L}}{\rightarrow} \mathcal{N}(0, \eta(m, 2) I Q), m>2 \\
\sqrt{N}\left[M P V_{N}(m, 4)-I Q\right] & \stackrel{\text { Stable } \mathcal{L}}{\rightarrow} \mathcal{N}\left(0, \eta(m, 4) \int_{0}^{1} \sigma_{u}^{8} d u\right), m>4
\end{array}
$$

where the known "efficiency factors" $\eta(m, p)$ are increasing in the block size $m$. Hence, low values of $m$ improve efficiency under the no-jump null hypothesis. Prominent examples of MPV estimators for IQ are the fourth order versions of the Tripower $(m=3)$, Quadpower $(m=4)$ and Quintpower $(m=5)$ variation estimators,

$$
\begin{aligned}
T Q_{N} & =M P V_{N}(3,4)=d_{3,4} \frac{N^{2}}{N-2} \sum_{i=1}^{N-2}\left|r_{i}\right|^{4 / 3}\left|r_{i+1}\right|^{4 / 3}\left|r_{i+2}\right|^{4 / 3} \\
M P Q_{N}(4) & =M P V_{N}(4,4)=d_{4,4} \frac{N^{2}}{N-3} \sum_{i=1}^{N-3}\left|r_{i}\right| \cdots\left|r_{i+3}\right| \\
M P Q_{N}(5) & =M P V_{N}(5,4)=d_{5,4} \frac{N^{2}}{N-4} \sum_{i=1}^{N-4}\left|r_{i}\right|^{4 / 5} \cdots\left|r_{i+4}\right|^{4 / 5}
\end{aligned}
$$

As noted, the lower order MPV estimators are more efficient than their higher order counterparts. On the other hand, they are less jump robust, in finite samples, and may lack a limiting distribution under the alternative as they employ fewer neighboring terms to dampen the jumps. Empirically, one therefore faces the familiar tradeoff between efficiency (under the no-jump null) and robustness to jumps (and other data imperfections) that will be comprehensively discussed below.

From the perspective of the blocking strategy, the basic MPV statistics operate with a relatively narrow window size of $m$ returns. However, in our empirical implementation, the individual returns are obtained via pre-averaging from a set of non-overlapping tick-by-tick returns so, depending on market liquidity, the windows for the higher order statistics may end up covering decidedly non-trivial time intervals.

\footnotetext{
${ }^{12}$ The distributional convergence is stable with a mixed Gaussian limit, i.e., a normal distribution conditional on the realization of the integrated power variation, $P V(q)=\int_{0}^{1} \sigma_{u}^{q} d u$, where, importantly, the limiting normal variate is independent of the (random) power variation process, and we have $q=4$ and $q=8$ in this case. See, e.g., Jacod and Shiryaev (2003), Chapter IX, for an introduction to the concept of stable convergence.
} 


\subsubsection{Truncated Power Variation Estimators}

A recent approach to robust IV (and IQ) estimation is the truncation based RV and BV estimators of Mancini (2009), further developed by CPR, which achieve jump robustness by truncating observations exceeding a pre-specified threshold. Under infill asymptotics, we may stipulate that the threshold converges toward zero slowly enough (slower than

$\left.\frac{1}{N} \log (N)\right)$ that the limiting distribution of the resulting estimators is identical to their non-truncated counterparts. In particular, the truncated RV is the most efficient among all jump robust IV estimators, and similarly the truncated RQ is the most efficient jump-robust estimator for IQ. Moreover, it is evident that the jump distortion is determined by the size of the truncation threshold and thus is under direct control in designing the estimator. The general form of the (symmetric) threshold multi-power variation estimator of order $m$ takes the form,

$$
\operatorname{TMPV}(p, m)=\mu_{p / m}^{-m} \frac{N^{\frac{p}{2}}}{N-m+1} \sum_{i=1}^{N-m+1} \prod_{j=0}^{m-1}\left|r_{i+j}\right|^{\frac{p}{m}} \boldsymbol{I}_{\left\{r_{i+j}^{2} \leq \vartheta_{i-j}\right\}} \stackrel{P}{\longrightarrow} \int_{0}^{1} \sigma_{u}^{p} d u
$$

Of course, in finite samples the choice of threshold matters and can be delicate. As noted by $\mathrm{CPR}$, it is beneficial to truncate aggressively to reduce the jump distortion by choosing a relatively low threshold. However this will also imply that non-jump returns are truncated with non-trivial probability. Hence, CPR suggest a finite sample upscaling to correct for the downward bias (under the no-jump hypothesis) induced by truncation of diffusive returns. Given the time variation in volatility, any such correction must rely on an estimate of the local volatility. Hence, they implement an iterative scheme to obtain a fixed point at which the expectation of the truncated estimator equals the true (estimated) volatility under the null hypothesis. While conceptually appealing, this approach has some drawbacks. First, the modified estimator is no longer linear in the unobserved $\sigma^{2}$ and thus suffers from a downwards bias, due to Jensen's inequality, even in the ideal Brownian case, because it requires inserting a local volatility estimate. Second, the approach uses a two-sided window of a given length (e.g., 50 observations) to arrive at the local volatility estimate, thereby rendering it susceptible to an additional downward bias due to time variation in volatility across the block. Both of these effects are evident in the simulation results reported in Section 4 .

\subsubsection{Nearest Neighbor Truncation Estimators}

Andersen, Dobrev and Schaumburg, henceforth ADS, (2009) introduced a couple of IV estimators designed to improve on the trade-off between jump robustness and efficiency confronting the MPV estimators while also allowing for a limiting theory under the jump 
alternative. The estimators are given as,

$$
\begin{aligned}
& \operatorname{MinRV}_{N}=d_{\mathrm{Min}, 2}\left(\frac{N}{N-1}\right) \sum_{i=1}^{N-1} \min \left(\left|r_{i}\right|,\left|r_{i+1}\right|\right)^{2} \\
& M \operatorname{MedRV} V_{N}=d_{\mathrm{Med}, 2}\left(\frac{N}{N-2}\right) \sum_{i=2}^{N-1} \operatorname{med}\left(\left|r_{i-1}\right|,\left|r_{i}\right|,\left|r_{i+1}\right|\right)^{2}
\end{aligned}
$$

where, for $Z_{i} \sim$ i.i.d. $\mathcal{N}(0,1), i=1,2,3$, the normalization constants are determined as, $d_{\mathrm{Min}, p}=\left(E\left[\min \left(\left|Z_{1}\right|^{p},\left|Z_{2}\right|^{p}\right)\right]\right)^{-1}$ and $d_{\mathrm{Med}, p}=\left(E\left[\operatorname{med}\left(\left|Z_{1}\right|^{p},\left|Z_{2}\right|^{p},\left|Z_{3}\right|^{p}\right)\right]\right)^{-1}$.

Under relatively weak conditions, the MinRV and MedRV estimators are consistent under the finite jump scenario and we may establish an associated asymptotic distribution theory which is independent of the finite activity jump process. Moreover, in finite samples, the distortion due to isolated jumps is of order $O(1 / N)$ versus the higher order $O\left(N^{-1+h}\right)$, for $h=\frac{p}{2 m}>0$, in the case of the MPV estimators.

As noted previously, the need to dampen the (finite sample) impact of jumps becomes increasingly acute as one estimates higher order power variation measures. The nearest neighbor truncation principle may be particularly advantageous in this context as isolated large jumps are effectively annihilated. Consequently, we extend the theory of nearest neighbor truncation to cover general integrated power variation estimation by developing the following jump-robust estimators,

$$
\begin{aligned}
& \operatorname{MinP}_{N}(p)=d_{\text {Min }, p}\left(\frac{N^{\frac{p}{2}}}{N-1}\right) \sum_{i=1}^{N-1}\left[\min \left(\left|r_{i}\right|,\left|r_{i+1}\right|\right)\right]^{p} \\
& \operatorname{MedPV}_{N}(p)=d_{\text {Med }, p}\left(\frac{N^{\frac{p}{2}}}{N-2}\right) \sum_{i=1}^{N-2}\left[\operatorname{med}\left(\left|r_{i}\right|,\left|r_{i+1}\right|,\left|r_{i+2}\right|\right)\right]^{p}
\end{aligned}
$$

and establish the following properties (proofs are relegated to the appendix):

Proposition 1 If Assumption 1 applies and $p$ is a positive, even integer then, as $N \rightarrow \infty$,

$$
\operatorname{MinP} V_{N}(p) \stackrel{P}{\longrightarrow} \int_{0}^{1} \sigma_{u}^{p} d u \quad \text { and } \quad \operatorname{MedPV}_{N}(p) \stackrel{P}{\longrightarrow} \int_{0}^{1} \sigma_{u}^{p} d u
$$

A corresponding asymptotic distribution theory is available,

Proposition 2 If Assumptions 1 and $1 A$ both apply then, for any positive, even integer, $p$,

$$
\begin{array}{ll}
\sqrt{N}\left(\operatorname{MinP} V_{N}(p)-\int_{0}^{1} \sigma_{u}^{p} d u\right) \stackrel{\text { Stable } \mathcal{L}}{\rightarrow} \mathcal{N}\left(0, \eta(\text { Min }, p) \int_{0}^{1} \sigma_{u}^{2 p} d u\right) \\
\sqrt{N}\left(\operatorname{MedPV} V_{N}(p)-\int_{0}^{1} \sigma_{u}^{p} d u\right) \stackrel{\text { Stable } \mathcal{L}}{\rightarrow} \mathcal{N}\left(0, \eta(\text { Med, } p) \int_{0}^{1} \sigma_{u}^{2 p} d u\right)
\end{array}
$$

where $\eta($ Min, $p)$ and $\eta($ Med, $p)$ are known constants. 
Moreover, these results remain valid for infinite activity jump processes in volatility subject to the regularity conditions of Barndorff-Nielsen, Shephard and Winkel (2006). ${ }^{13}$

Of course, our main focus is Integrated Quarticity (IQ) estimation. Proposition 2 provides the following realized quarticity estimators for the case of $p=4$,

$$
\begin{aligned}
& \operatorname{MinRQ}_{N}=\operatorname{MinPV}_{N}(4)=d_{\mathrm{Min}, 4}\left(\frac{N^{2}}{N-1}\right) \sum_{i=1}^{N-1}\left[\min \left(\left|r_{i}\right|,\left|r_{i+1}\right|\right)\right]^{4} \\
& M e d R Q_{N}=\operatorname{MedPV} V_{N}(p)=d_{\mathrm{Med}, 4}\left(\frac{N^{2}}{N-2}\right) \sum_{i=1}^{N-2}\left[\operatorname{med}\left(\left|r_{i}\right|,\left|r_{i+1}\right|,\left|r_{i+2}\right|\right)\right]^{4} \text {. }
\end{aligned}
$$

Proposition 2 mirrors the existing limit theory for the MPV statistics. Table 1 provides the efficiency factors for some select estimators. Panel A demonstrates, as is well known, that the RV estimator is most efficient, followed by low order MPV estimators. Estimation of IV by MinRV entails a significant increase in the asymptotic variance relative to, say, $\mathrm{BV}$, while MedRV is much closer to matching the efficiency of BV. Likewise, Panel B shows that MedRQ is more efficient than quadpower, $M P Q 4=M P V(4,4)$, in estimating IQ. These observations suggest that MedRV and MedRQ will outperform all MPV estimators that allow for an asymptotic distribution theory under the jump alternative, as the latter correspond to $\operatorname{MPV}(m, 2)$ for $m \geq 3$ in the case of $\operatorname{IV}$ and $\operatorname{MPV}(m, 4)$ for $m \geq 5$ in the case of IQ. In addition, the nearest neighbor truncation estimators have the advantage of near elimination of finite sample distortion stemming from isolated price jumps. Finally, from the perspective of the blocking strategy, the MinPV and MedPV estimators operate with narrow window widths of $m=2$ and $m=3$, respectively. Thus, they are highly localized estimators which equips them with robustness towards time variation in volatility, matching the relatively favorable features of the bipower and tripower variation measures in this regard. As such, MedRV and MedRQ may be potentially attractive candidates for jump-robust inference and jump test applications.

Finally, we note that the nearest neighbor truncation estimators are limited in their ability to adapt to shifts in the underlying efficiency-robustness trade-off due to the use of very small return blocks. The following section provides a generalization of the underlying estimation principle to enable added flexibility in dealing with different orders of return power variation.

\subsection{Robust Neighborhood Truncation Estimation}

The nearest neighbor truncation approach selects a specific order statistic for absolute returns on a small block to obtain an unbiased power variation estimator under the null

\footnotetext{
${ }^{13}$ These conditions become increasingly stringent as the order of the power variations rises, so we do not discuss this potential extension any further.
} 
Table 1: Comparison of efficiency of selected multi power variation and nearest neighbor truncation estimators. Panel A: Efficiency factors (from best to worst) for $\operatorname{IV}, \eta(\cdot, 2)$. Panel B: Efficiency factors for IQ, $\eta(\cdot, 4)$.

\begin{tabular}{|c|c|c|c|c|c|c|c|c|c|}
\hline \multirow[t]{2}{*}{ Panel A: } & $\mathrm{RV}$ & BV & MedRV & TV & MPV4 & MPV5 & MPV6 & MinRV & MPV7 \\
\hline & 2.00 & 2.65 & 2.96 & 3.06 & 3.38 & 3.61 & 3.78 & 3.81 & 3.91 \\
\hline \multirow[t]{2}{*}{ Panel B: } & & $\mathrm{RQ}$ & BQ & TQ & MedRQ & MPQ4 & MPQ5 & MinRQ & \\
\hline & & 10.66 & 12.00 & 13.65 & 14.16 & 14.92 & 15.85 & 18.54 & \\
\hline
\end{tabular}

of constant local volatility. This set-up facilitates the design of simple strategies to ensure robustness in directions of particular concern. However, the relative importance of factors impacting the trade-off between statistical efficiency and robustness changes substantially as we move from estimating the integrated return variation to higher order power variation. Hence, the most suitable design for the integrated return variation, or $p=2$, is unlikely to be preferable when estimating the integrated quarticity, or $p=4$. Consequently, we now extend the nearest neighbor truncation principle to allow for a larger block size and an enhanced menu of inference procedures based on order statistics.

\subsubsection{Neighborhood Truncation Estimators}

Before presenting the general estimator we introduce some useful notation. First, we denote the $i^{\text {th }}$ block of absolute returns $\underline{r}_{i, m}=\left(\left|r_{i}\right|, \cdots,\left|r_{i+m-1}\right|\right), i=1, \ldots, N-m+1$. Next, $q_{j}\left(\left|r_{1}\right|, \ldots,\left|r_{m}\right|\right),=q_{j}\left(\underline{r}_{i, m}\right), j=1, \cdots, m$, is the $j^{\text {th }}$ order statistic of the $i^{\text {th }}$ absolute return block, so $q_{1}\left(\underline{r}_{i, m}\right) \leq \ldots \leq q_{m}\left(\underline{r}_{i, m}\right)$. Under the null hypothesis that these returns are i.i.d. $\mathcal{N}\left(0, \sigma_{i}^{2} / N\right)$, we may also write $N^{\frac{p}{2}} \underline{r}_{i, m}=\sigma_{i} \underline{Z}_{i, m}$, highlighting the fact that all observations and estimators for the block ultimately are functionals operating on the realization of an $m$-dimensional standard normal random vector.

In this setting, we naturally have $m$ separate unbiased estimators for $\sigma_{i}^{p}$, namely one for each order statistic. We denote these "Neighborhood Truncation" estimators, or $N T_{N}^{(j, m)}(p)$. In analogy with the MinPV and MedPV estimators, they take the form,

$$
N T_{N}^{(j, m)}(p)=d_{(j, m)}(p) \cdot\left(\frac{N^{\frac{p}{2}}}{N-m+1}\right) \sum_{i=1}^{N-m+1}\left[q_{j}\left(\underline{r}_{i, m}\right)\right]^{p}, \quad j=1, \ldots, m
$$

where the scaling factor $d_{(j, m)}(p)=\left(E\left[q_{j}\left(\left|Z_{1}\right|^{p}, \cdots,\left|Z_{m}\right|^{p}\right)\right]\right)^{-1}, Z_{i} \sim$ i.i.d. $\mathcal{N}(0,1), \quad i=$ $1, \ldots, m$, ensures that the scaled $p^{\text {th }}$ power of the $j^{\text {th }}$ absolute return order statistic provides an unbiased estimator for $\sigma^{p}$. Since these scaling factors are obtained as the inverse 
of a set of order statistics we have the ranking, $d_{(1, m)}(p)>\ldots>d_{(m, m)}(p) .{ }^{14}$

This class provides a direct extension of the original nearest neighbor truncation estimators as we have, $\operatorname{MinP} V(p)=N T_{N}^{(1,2)}(p)$ and $\operatorname{MedPV}(p)=N T_{N}^{(2,3)}(p)$, with scaling factors, $d_{M i n}(p)=d_{(1,2)}(p)$, and $d_{\text {Med }}(p)=d_{(2,3)}(p) \cdot{ }^{15}$

If we focus on a single return block, $\underline{r}_{i, m}$, for a given power variation estimator, we may consider $N, m$, and $p$ fixed, and we will suppress either of these indices whenever convenient as long as their exclusion does not lead to ambiguity. Under the block null, we then define the neighborhood truncation estimator based on the $j^{\text {th }}$ order statistic applied to a given return block as,

$$
\mathcal{E}_{j}=d_{(j, m)}(p) \cdot N^{\frac{p}{2}} \cdot\left[q_{j}\left(\underline{r}_{i, m}\right)\right]^{p}=\sigma_{i}^{p} \cdot d_{(j, m)}(p) \cdot\left[q_{j}\left(\underline{Z}_{i, m}\right)\right]^{p} .
$$

If we want to be explicit about the estimator referring to the $i^{\text {th }}$ return block, we may loosely write $\mathcal{E}_{j}=\mathcal{E}_{j}\left(\underline{r}_{i, m}\right)$, or even $\mathcal{E}_{j}=\mathcal{E}_{j}\left(\underline{Z}_{i, m}\right)$. Under the maintained null, the set of unbiased neighborhood truncation estimators for $\sigma_{i}^{p}$ is summarized by, $\mathcal{E}=$ $\left\{\mathcal{E}_{1}, \ldots, \mathcal{E}_{m}\right\} .{ }^{16}$

In this notation, picking a specific NT estimator amounts to, a priori, selecting a given integer $j$ from the set $\{1, \cdots, m\}$. However, viewed from this perspective, it is natural to explore whether estimators with superior properties may be obtained via, say, a linear combination of NT estimators. ${ }^{17}$ To formalize this idea, we introduce a "selector index" vector which identifies the order statistics used in the construction of a given estimator. Hence, we let $\boldsymbol{I}=\left(k_{1}, \ldots k_{H}\right), 1 \leq H \leq m$, be a $1 x H$ vector consisting of an ordered subset of integers from $\{1, \cdots, m\}$, where the integer $k_{h}, h=1, \cdots, H$, denotes the $h^{\text {th }}$ order statistic from the original block to be included in the selection. Hence, the final estimator exploits the candidate set $\left\{\mathcal{E}_{k_{1}}, \cdots \mathcal{E}_{k_{H}}\right\}$. For example, $\mathcal{E}_{j}$ is obtained by stipulating $\boldsymbol{I}=\{j\}$, while estimators exploiting the joint information in several NT estimators specifies $\boldsymbol{I}$ as a vector with multiple indices. The total number of distinct non-empty subsets of $\{1, \cdots, m\}$ is $2^{m}-1$. Denoting the set of all possible selector index vectors $\mathcal{I}$, a specific combination of estimators is uniquely identified by the vector, $\boldsymbol{I} \in \mathcal{I}$, ranging from

\footnotetext{
${ }^{14}$ For $p \geq 4$ and $m \geq 3$, these scaling factors become quite large for the lower order statistics and very small for the higher ones; e.g., for IQ estimation based on order statistics for a block with five returns, we obtain $d_{(1,5)}(4)=35.0, d_{(2,5)}(4)=5.74, d_{(3,5)}(4)=1.44, d_{(4,5)}(4)=0.40, d_{(5,5)}(4)=0.086$, see Table 10 in the appendix.

${ }^{15}$ For the integrated variance $(p=2)$, these estimators may be interpreted as a redesigned version of the original Quantile Realized Variation, or QRV, estimator of Christensen, Oomen and Podolskij (COP) (2008), where the QRV estimator is adapted to quantiles based on absolute rather than raw returns. The adaptation of QRV to cover the ADS (2009) nearest neighbor truncation estimators is discussed in COP (2010).

${ }^{16}$ In this generic notation it is evident that $\mathcal{E}$ may designate an arbitrary set of $m$ ordered, unbiased $\sigma_{i}^{p}$ estimators obtained as functionals of the underlying return block. Many of the insights below will continue to apply in this general context.

${ }^{17}$ This is analogous to the use of such linear combinations for the QRV estimator by COP (2008).
} 
one-dimensional vectors, identifying the original NT estimators at the one extreme, to the full vector $\{1, \cdots, m\}$ indicating the use of all candidate estimators, at the other extreme.

It is evident that superior asymptotic performance will be obtained by combining, and thus exploiting the information associated with, all the estimators as long as we remain within the ideal setting of Assumptions 1 and 1A. However, this must be weighed against the robustness objective of ensuring reliable inference in finite samples in the presence of jumps as well as other potential sources of noise. In fact, such robustness concerns motivate us to consider an even broader class of alternative estimators, leading to the "Robust Neighborhood Truncation," or RNT, principle to which we now turn.

\subsubsection{Robust Neighborhood Truncation Estimators}

This section develops a procedure for robust estimation of integrated power variation based on order statistics of a set of unbiased local power variation estimators on a block of returns. In principle, this original set of estimators may be obtained by a variety of functionals and estimation strategies, and need not originate from the neighborhood truncation approach. The general features of our discussion will remain similar for such scenarios. Below, however, we focus on the case where the initial set consists exclusively of NT estimators. As for the basic neighborhood truncation estimators, the idea is to obtain robustness by relying on a scaled order statistic, but now applied in a second step to the candidate unbiased estimators, and not the underlying absolute returns. Since this involves a second layer of order statistics, the notation is necessarily more complex, and we first introduce some useful conventions.

First, as indicated in the preceding section, for each return block we rely on a set of $H$ unbiased candidate estimators for $\sigma^{p}$ obtained via the corresponding order statistics, $\left\{\mathcal{E}_{k_{1}}, \cdots \mathcal{E}_{k_{H}}\right\}$. It is evident that the appropriate scaling factor, converting an order statistic, applied to such a set of unbiased $\sigma^{p}$ estimators, into a new unbiased (and robust) estimator, will depend on the specific composition of the set. Hence, the definition of this scaling factor must reference the underlying NT estimators explicitly. Consequently, we denote them $d_{(j, I)}(p)$. They are often not available in closed form, but they may be characterized in terms of functionals of independent standard normal variables which allows for straightforward determination by computational means.

Second, combining the above conventions, we may define the robust neighborhood truncation estimator based on the $j$ 'th order statistic among the unbiased candidate estimators, as $d_{(j, \boldsymbol{I})}(p) \cdot q_{j}\left(\mathcal{E}_{k_{1}}, \cdots, \mathcal{E}_{k_{H}}\right)$. Written more explicitly, this becomes,

$$
d_{(j, I)}(p) \cdot N^{\frac{p}{2}} \cdot q_{j}\left[d_{\left(k_{1}, m\right)}(p) \cdot q_{k_{1}}\left(\underline{r}_{i, m}^{p}\right), \cdots, d_{\left(k_{H}, m\right)}(p) \cdot q_{k_{H}}\left(\underline{r}_{i, m}^{p}\right)\right]
$$

We may then define the Robust Neighborhood Truncation estimator obtained from the 
$j$ 'th order statistic of the candidate set as,

$$
R N T_{N}^{(j, \boldsymbol{I})}(p)=d_{(j, \boldsymbol{I})}(p) \frac{1}{(N-m+1)} \sum_{i=1}^{N-m+1} q_{j}\left[\mathcal{E}_{k_{1}}\left(\underline{r}_{i, m}\right), \cdots, \mathcal{E}_{k_{H}}\left(\underline{r}_{i, m}\right)\right]
$$

where the scaling factor is given by,

$$
d_{(j, \boldsymbol{I})}(p)=\left(E\left[q_{j}\left(d_{\left(k_{1}, m\right)}(p) Z_{\left(k_{1}, m\right)}^{p}, \cdots, d_{\left(k_{H}, m\right)}(p) Z_{\left(k_{H}, m\right)}^{p}\right)\right]\right)^{-1},
$$

and $Z_{(k, m)}$ denotes the $k$ 'th absolute order statistic from a block of $M$ i.i.d. $\mathcal{N}(0,1)$ random variables. While the scaling factor, $d_{(j, I)}(p)$, generally is not available in closed form it is readily determined, to an arbitrary degree of precision, by simulation. As before, we achieve jump robustness by constructing an estimator that avoids using the largest order statistic among the available set.

By analogous arguments to those for the nearest neighbor truncation estimators, the RNT estimator is consistent.

Proposition 3 If Assumption 1 applies and $p$ is a positive, even integer then, as $N \rightarrow \infty$,

$$
R N T_{N}^{(j, \mathbf{I})}(p) \stackrel{P}{\longrightarrow} \int_{0}^{1} \sigma_{u}^{p} d u, \quad j=1, \cdots, H .
$$

In addition, the associated asymptotic distribution theory is similar.

Proposition 4 If Assumptions 1 and $1 A$ both apply then, for any positive, even integer, $p$,

$$
\sqrt{N}\left(R N T_{N}^{(j, \mathbf{I})}(p)-\int_{0}^{1} \sigma_{u}^{p} d u\right) \stackrel{\text { Stable } \mathcal{L}}{\rightarrow} \mathcal{N}\left(0, \eta(j, \mathbf{I} ; p) \int_{0}^{1} \sigma_{u}^{2 p} d u\right), \quad j=1, \cdots, H
$$

where $\eta(j, \mathbf{I} ; p)$ is a known constant.

This result encompasses the Neighborhood Truncation and Nearest Neighbor Truncation estimators as special cases as the latter involve a specific choice for the vector $\boldsymbol{I}$. Consequently, this constitutes a new class of estimators that may compare favorably to existing inference procedures for integrated return power variation in some dimensions. In particular, the application of a sequence of order statistics offer great flexibility in accommodating specific robustness features of concern in a given application. Of course, the leading applications of RNT will be for estimation of integrated return variation and integrated return quarticity. Hence, whenever there is no concern about ambiguity, we often suppress the additional indices and refer to the RNT(2) estimator as RNTV and RNT(4) as RNTQ, so that the notation is better aligned with the one usually adopted for these orders of integrated power variation. 
The simulation and empirical work in the following sections exploit fairly small blocks of $M \leq 5$ in order to retain resiliency relative to rapidly changing volatility levels during the trading day. In addition, the highest order statistics among the set $\boldsymbol{I}$ are excluded to achieve a reasonable degree of jump robustness. Finally, we also find it useful to eliminate estimators that stem from the lowest order statistics of the underlying returns as these are relatively more affected by market microstructure noise such as price discreteness and bid-ask bounce. In addition, these estimates obtain unbiasedness by scaling the original small returns by a large factor, implying that any potential microstructure features are aggravated. Hence, we typically exclude $\mathcal{E}_{1}$, and often also $\mathcal{E}_{2}$, from $\boldsymbol{I}$. Figure 3 exemplifies the construction of RNTQMin5, our simplified notation for the $R N T Q^{1,(3,4,5)}$ estimator, which plays a prominent role in our analysis in Sections 4 and 5 .

\section{(1) Retains three largest absolute returns}

(2) Raises the returns to $4^{\text {th }}$ power

(3) Scales each to an unbiased estimator of $\sigma^{4}$

(4) Selects suitable low order quantile (e.g. Min)

(5) Scales result to yield unbiased final estimate
RNTQMin5

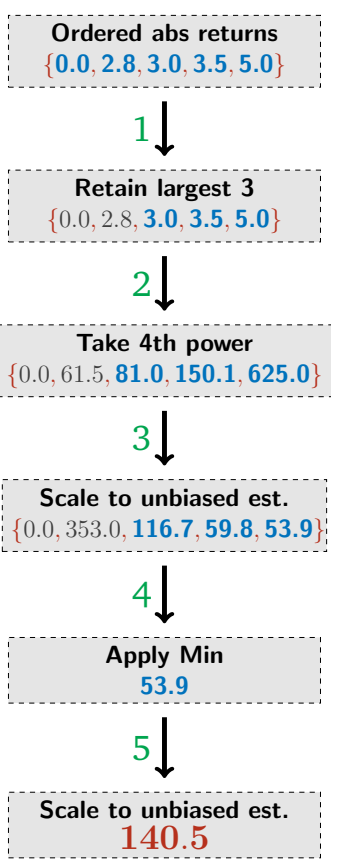

Figure 3: Schematic representation of the construction of the RNTQMin5 estimator of $\sigma^{4}$ on a block of five adjacent returns.

The example in Figure 3 warrants a few comments. First, for illustration, we display the estimators obtained from the first and second return order statistics along with the rest, even if the former are excluded from the ultimate construction of the estimator. As such, the third box from the top displays the five fourth order returns that would have been included in the standard RQ estimator. The extreme right skew of the components is evident, spanning 0 to 625 , even if the initial returns do not appear particularly scattered. The zero return is, of course, fairly common due to the discreteness of the price grid. 
Second, the step between box 3 and 4 reflects the application of the relevant scaling factors for the set of neighborhood truncation estimators, $N T_{N}^{(j, 5)}(4)$, which is given by $d_{(j, 5)}(4)$ for $j=1, \ldots, 5$, explicitly listed in footnote 14 . In particular, the large scaling factor $(5.74)$ associated with the fourth power of the second order absolute return statistic produces the, by far, largest realized unbiased estimator among the set in box four (353) at more than three times the second largest estimator. Finally, excluding the NT estimators stemming from the two lowest absolute returns $(0,353)$, we pick the minimum of the remainder and upscale this first order statistic from the subset of unbiased estimators (53.9) suitably to obtain this particular local RNT estimator of $\sigma^{4}$ of 140.5. In the end, this specific functional realization is based on the largest order statistic from the original block of returns (5.0), but the low scaling factor of (the fourth power of) this return $(0.086$, as indicated in footnote 14) implies that the associated (unbiased) NT estimator in box four is the smallest of the relevant estimators. Third, the appropriate scaling factor for this smallest order statistic among the given set of candidate estimators is then, $d_{(j, \boldsymbol{I})}(p)=d_{(1,(\mathbf{3}, \mathbf{4}, \mathbf{5})}(4)$, which is readily determined by simulation under the null hypothesis. Overall, the final local estimate of 140.5 falls squarely in the middle to upper ranges of the potential candidate RQ style estimators in box 3 and NT estimators in box 4, as seems natural when constructing a robust estimator based on a set of initial estimators that are drawn from a severely right skewed distribution.

Finally, we note that the second layer of order statistics associated with the RNT estimators involves a nonlinear transformation that takes them outside the basic class of NT or QRV estimators. Moreover, as we document below, it embeds them with properties that typically are very distinct from, and often preferable to, those obtained by existing high order integrated power variation estimators in dimensions of practical interest.

\subsection{Using the Ratio $\frac{\sqrt{\widehat{I Q}}}{\widehat{I V}}$ for Robust IV Inference}

The primary applications of IQ estimation is to draw inference about IV and to test for the presence of jumps under the null hypothesis of no-jumps. For these procedures to perform well, it is essential to have estimators of IV and, especially, IQ with good efficiency and finite sample jump robustness.

Let $\widehat{I V}, \widehat{I Q}$ be suitable jump-robust estimators of IV and IQ within, say, the MPV, nearest neighbor truncation, or truncation MPV estimator class. Then the natural approach for drawing inference about IV is based on the limiting distribution of:

$$
\frac{\sqrt{N}[\widehat{I V}-I V]}{\sqrt{\eta \widehat{I Q}}} \stackrel{\text { Stable } \mathcal{L}}{\rightarrow} \mathcal{N}(0,1)
$$

where $\eta$ is a scaling factor that depends on the specific choice of estimator.

Letting RV denote the realized volatility estimator, which is the efficient estimator of 
IV under the null, the natural Hausman test statistic for the presence of jumps, see BNS (2004) and Huang and Tauchen (2005), is given by

$$
\frac{\sqrt{N}[\widehat{R V}-\widehat{I V}]}{\sqrt{(\eta-2) \widehat{I Q}}} \stackrel{\text { Stable } \mathcal{L}}{\rightarrow} \mathcal{N}(0,1) .
$$

An asymptotically equivalent set of test statistics with better finite sample properties, proposed by BNS (2002), can be derived by applying the delta method to the log-transform of the volatility measures. This has the benefit that IQ enters only in terms of the ratio $\sqrt{I Q} / I V$ which, as also demonstrated in our empirical investigation below, has a stabilizing effect on the variance of $\log \widehat{I V}$.

$$
\frac{\sqrt{N}[\log (\widehat{I V})-\log (I V)]}{\sqrt{\frac{\eta \widehat{I Q}}{(\widehat{I V})^{2}}}} \stackrel{\text { Stable } \mathcal{L}}{\rightarrow} \mathcal{N}(0,1) .
$$

The corresponding Hausman test statistic for the presence of jumps is

$$
\frac{\sqrt{N}[\log (\widehat{R V})-\log (\widehat{I V})]}{\sqrt{(\eta-2) \frac{\widehat{I Q}}{(\widehat{I V})^{2}}}} \stackrel{\text { Stable } \mathcal{L}}{\rightarrow} \mathcal{N}(0,1) .
$$

In fact, the simulation and empirical results below are sufficiently compelling that we advocate using this ratio for jump-robust inference concerning integrated return variation. However, reliance on this ratio will also affect the way market microstructure noise impacts our inference. While it is impossible to provide a complete answer regarding the degree of noise robustness of the ratio statistic - it will hinge on the specific estimator and the nature of the noise - we find suggestive evidence pointing towards the favorable properties of the ratio statistic relative to the raw statistic in this dimension as well. The intuition is, as before, that particular biases tend to manifest themselves simultaneously in the IQ and IV statistics so that there is a partial cancelation of errors. The appendix provides an illustration of this point through computations involving the non-robust versions of the IQ and IV estimators which allow for relatively simple analytical expressions. The issue is further pursued within the simulation set-up entertained in the following section.

\section{Finite Sample Simulation Evidence}

We carefully design a series of Monte Carlo experiments, each focusing on a distinct feature of the data generating process that may affect the finite sample behavior of the various IV, IQ and $\sqrt{I Q} / I V$ (ratio) estimators. In particular, we compare the performance 
of pre-averaged RNT to different truncation, nearest neighbor and multi-power variation estimators for alternative simulation designs representing distinct stylized features of the intraday return series. The emphasis is on understanding the qualitative impact of each feature in isolation. In reality, multiple features are interacting simultaneously, creating rather complex patterns in tick-by-tick data. The joint presence of various factors, which partially offset or reinforce the effect of each other, render it difficult to infer the significance and impact of individual features. Hence, our simulation designs do not intend to replicate the empirical results in all dimensions, but rather to assist in identifying what market characteristics are plausible candidates for explaining the systematic patterns we observe in performance across the alternative estimators.

\subsection{Estimators}

We adopt the novel filtering procedure, based on (mildly) truncating the local power variation functional, for all the estimators except those already truncating individual returns more aggressively, i.e., the truncation power variation estimators, TRV, TBV, TRQ, and TBQ. For the latter, the impact of an additional layer of mild truncation is negligible so leaving these estimators intact allows for a more direct comparison of the alternative approaches. ${ }^{18}$ In implementing the truncation estimators, we adopt the CPR procedure as a benchmark as they explore alternative versions of these estimators in some depth. As mentioned, all other estimators are subjected to the mild truncation of the local estimating functional. This set of IV and IQ estimators include,

1. Multi-power variation benchmark estimators: MVP3, MPV4 and MPV5, as well as MPQ3, MPQ4 and MPQ5. This type of estimators was first considered by BNS (2004);

2. Nearest neighbor truncation estimators: NTVMin2 $=N T V^{1,2} \equiv M i n R V$ and NTVMed3 $=$ $N T V^{2,3} \equiv M e d R V$, along with NTQMin2 $=N T Q^{1,2} \equiv \operatorname{MinRQ}$ and NTQMed3 = $N T Q^{2,3} \equiv M e d R Q$. These are developed by ADS (2009) and in this paper;

3. Robust Neighborhood Truncation estimators: RNTVMin2 $=R N T V^{1,(1,2)}, \mathrm{RNTVMed} 3=$ $R N T V^{2,(1,2,3)}$, RNTVMin5 $=R N T V^{1,(3,4,5)}$, and RNTVMed5 $=R N T V^{2,(3,4,5)}$, as well as RNTQMin2 $=R N T Q^{1,(1,2)}$, RNTQMed3 $=R N T Q^{2,(1,2,3)}$, RNTQMin5 = $R N T Q^{1,(3,4,5)}$, and RNTQMed5 $=R N T Q^{2,(3,4,5)}$, as developed in the current paper.

In addition, in our analysis of IV estimators, we include the standard (non-truncated and non-functional filtered) RV estimator, serving as a non jump-robust benchmark, along with

\footnotetext{
${ }^{18}$ We confirm that our filtering procedure under the BM null, applied to pre-averaged returns, only is active for about 1 -in- $10^{6}$ return blocks, while the filter of CPR is applied to roughly 1 -in- $10^{3}$ returns on average.
} 
the QRV estimator of COP (2010). We omit an IQ counterpart of QRV from our analysis because we find the block size of 20 or more returns, necessary in order to establish the quantiles, to be prohibitively large for reliable inference on actual data that are subject to typical imperfections such as irregular sampling intervals and pronounced intraday variation in volatility. In addition, as discussed previously, one may interpret our nearest neighbor estimators as modified QRV estimators based on the absolute returns over very small blocks, while the new RNT class of estimators is inherently different.

Finally, taking into account the need to apply suitable noise-reduction technique when using the estimators in practice, we focus our Monte Carlo analysis exclusively on the preaveraged implementation of all estimators, as defined in more detail in appendix $\mathrm{C}$ and further discussed in section 4.2.4 below.

\subsection{Simulation Results}

We largely follow the comprehensive simulation design in Andersen et al (2009) adopted for comparing IV estimators. We calibrate the unconditional daily IV to 0.000159 , or roughly $20 \%$ per year, across all scenarios. For each scenario we simulate 250,000 trading days, corresponding to about 1,000 years, from 9:30 am to 4:00 pm with new prices arriving every 3 seconds on average, so we have 7,800 distinct prices each day.

We consider three major types of departure from the Gaussian benchmark: (i) jumps in returns: these stem from one or multiple intraday price jumps; (ii) time-varying volatility: stochastic and deterministic (diurnal pattern) variation in volatility along with volatility jumps; (iii) market microstructure noise: bid-ask bounce, price recording errors, irregular trade intervals, and price decimalization (discreteness). Each scenario is briefly described below, with additional details available in Andersen et al. (2009). In each case we focus on estimators of $I Q, I V$, and the ratio $\sqrt{I Q} / I V$, where the latter is calculated using the same type of estimator in both numerator and denominator. ${ }^{19}$ Our findings for IV are summarized in Tables 2-3, those for IQ are reported in Tables 4-5, while the ones for $\sqrt{I Q} / I V$ appear in Tables 6-7.

\subsubsection{Brownian motion: The "BM" baseline scenario}

This is our baseline Brownian motion model with sampling on an equispaced time grid. It is an ideal setting under which the finite sample performance of all estimators should mimic the underlying asymptotic theory. The results in Tables 2-7, Panel A, reveal that all estimators are unbiased at the higher frequencies and the ordering of estimators by finite sample RMSEs is as expected. Nonetheless, it is striking that TBV and TRV are the only

\footnotetext{
${ }^{19}$ Although it may, in theory, be preferable to employ a different type of estimators for the numerator and denominator, our experimentation points towards significant gains in retaining estimators within the identical class, as this facilitates more effective cancelation of noise and outliers.
} 
IV estimators suffering from a minor downward bias at the lower frequencies in Tables 2-3, Panel A, and likewise, TBQ and TRQ are the only IQ estimators in Tables 4-5, Panel A, that are mildly downward biased in the BM scenario for the lower frequencies. These biases stem from estimation noise in the truncation and recursive bias-correction of the estimators. Since the individual returns are truncated at three (estimated) standard deviations, unbiasedness necessitates an upward adjustment to mitigate the impact of occasional erroneous truncations of diffusive returns but this involves some degree of error, even under the BM null. By contrast, all remaining MPV, NT and RNT estimators are almost perfectly unbiased under the BM null. This reflects the much more conservative truncation level (p-value of $10^{-6}$ ) adopted when filtering outliers among the individual functional estimators of local power variation. For the ratio $\sqrt{I Q} / I V$ (true value equal to unity for BM), Tables 6-7, Panel A, reveal a slightly more systematic downward bias across all estimators from about the three minute sampling interval and increasing at the lower frequencies. This stems from a finite sample Jensen effect, impacting all estimators to a similar degree. ${ }^{20}$

In accordance with the asymptotic theory, TRV and TRQ are superior in terms of (RMSE) efficiency under the BM null. However, the RNTVMin5 and RNTQMin5 estimators are close to TRV and TRQ and are the best of the remaining estimators. As such, the behavior of these estimators under the various alternative scenarios, and in particular the jump setting, will be important in assessing their overall standing.

Finally, and importantly, when estimating the ratio $\sqrt{I Q} / I V$, the pairing of RNTVMin5 and RNTQMin5 significantly outperforms all other estimators, including the ratio based on TRQ and TRV (Panel A, Tables 6-7). The efficiency gains associated with the RNTQ/RNTV estimators reflect a more effective dampening of outliers in both the numerator and denominator of the ratio statistic, attainable through the specific design of the RNT estimators.

\footnotetext{
${ }^{20}$ The improved performance of the TBQ-TBV ratio is due to a fortuitous cancelation of the biases of the individual terms in numerator and denominator under the BM null.
} 


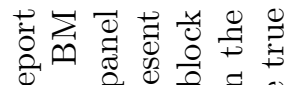
is $3 \mathbb{E}^{\circ}$

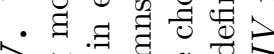
$\geq \infty$ on 녕 ह0 궁 웡 0

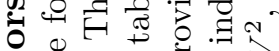

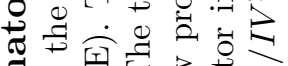

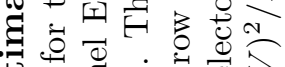
क $b_{0}$ on 总

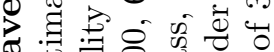
d

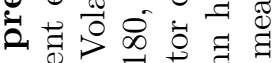

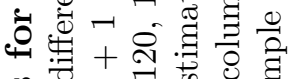

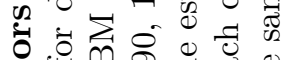

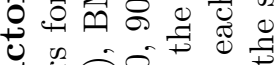

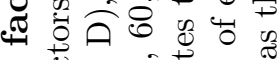

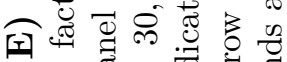

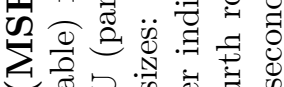

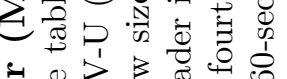

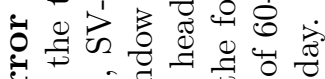
웡을

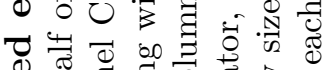
政

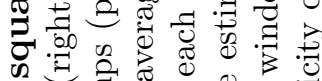

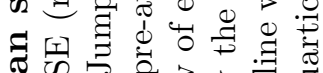

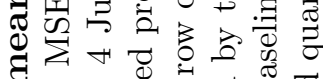

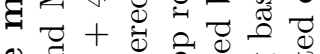

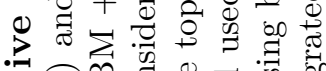

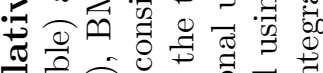

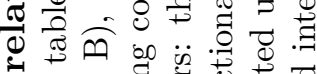

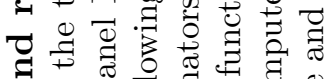

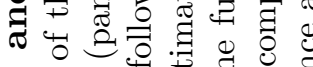
g 을

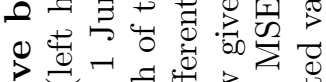

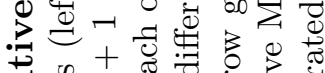

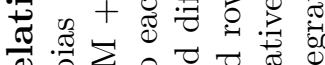

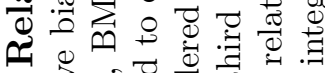

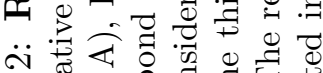

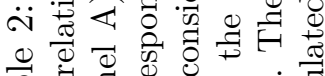

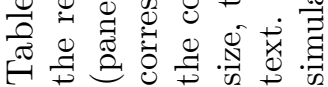

\begin{tabular}{|c|c|c|c|c|}
\hline & & & & \\
\hline 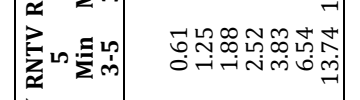 & 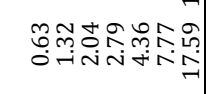 & 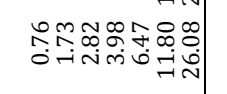 & 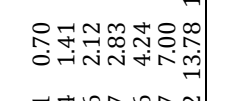 & 売 \\
\hline : & 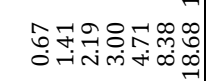 & 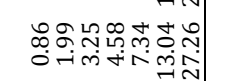 & 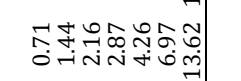 & \\
\hline 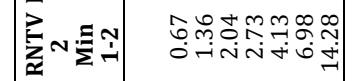 & 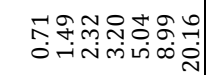 & 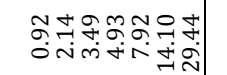 & 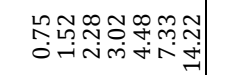 & \\
\hline 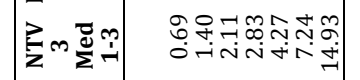 & 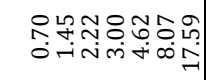 & 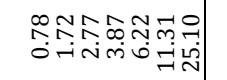 & 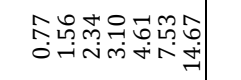 & \\
\hline 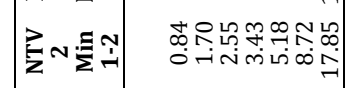 & & 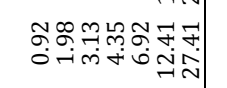 & 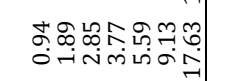 & \\
\hline 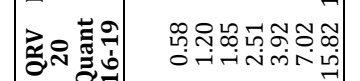 & 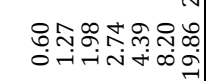 & 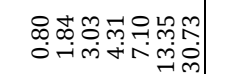 & 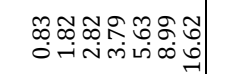 & \\
\hline 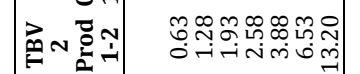 & 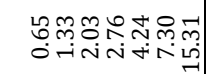 & 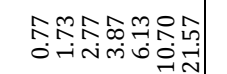 & 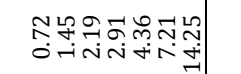 & \\
\hline 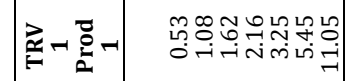 & 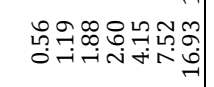 & 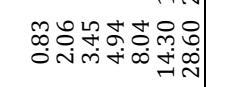 & 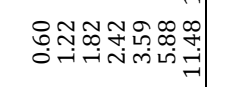 & \\
\hline 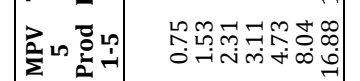 & 象: & 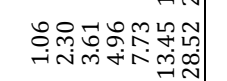 & 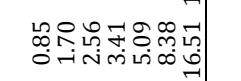 & \\
\hline & & 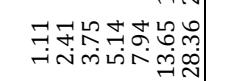 & & \\
\hline 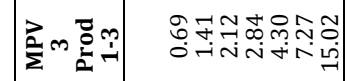 & 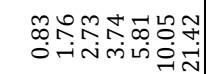 & 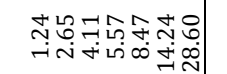 & 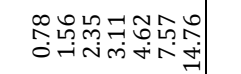 & \\
\hline 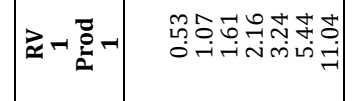 & 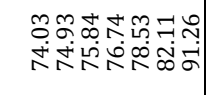 & 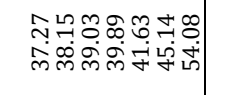 & 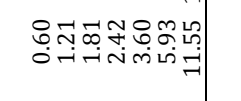 & \\
\hline 8 & 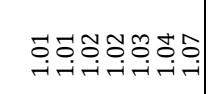 & 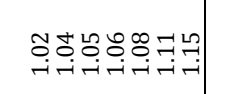 & 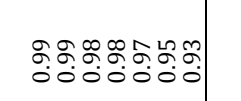 & \\
\hline : & 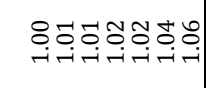 & 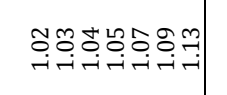 & 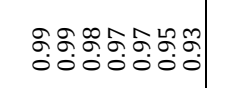 & \\
\hline 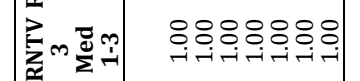 & 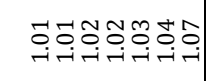 & 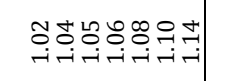 & 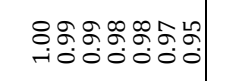 & \\
\hline కి: & 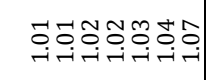 & stot & 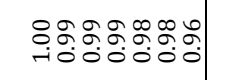 & \\
\hline 을요 & 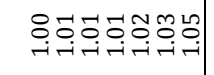 & 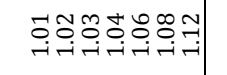 & 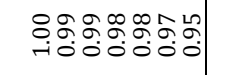 & \\
\hline & 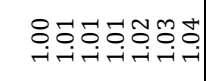 & 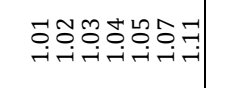 & 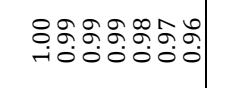 & \\
\hline 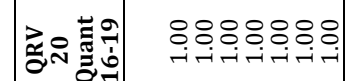 & 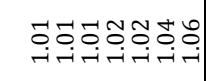 & 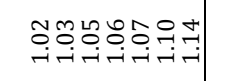 & 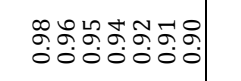 & \\
\hline : & : & 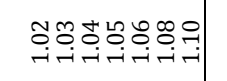 & 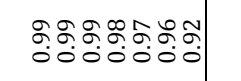 & \\
\hline 年 & 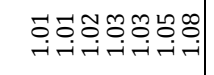 & 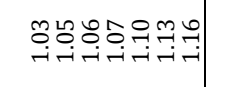 & 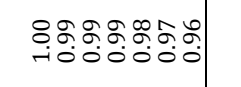 & \\
\hline & & 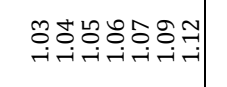 & 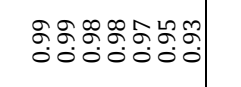 & \\
\hline కిะి: & & 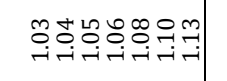 & 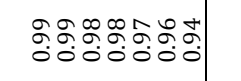 & \\
\hline & & 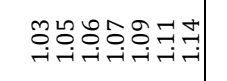 & 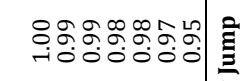 & \\
\hline & & 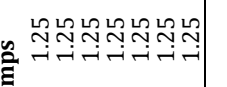 & 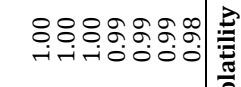 & 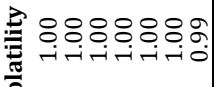 \\
\hline & & & 80. & \\
\hline & & & & \\
\hline
\end{tabular}




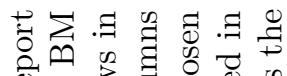
낭

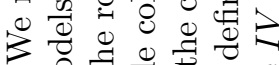

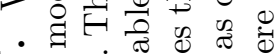

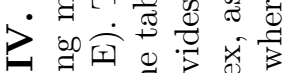
猔宁

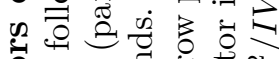
0 잉 0

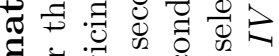
. 设之。

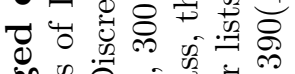

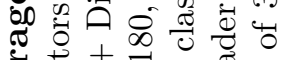
एँ

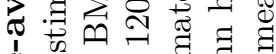

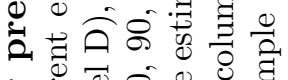

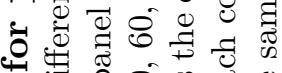

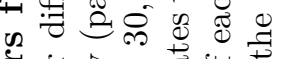

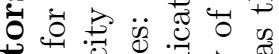
娄 $0 \begin{gathered}0 \\ 0\end{gathered}$

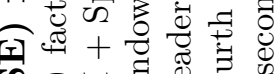

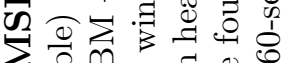

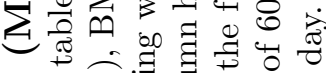

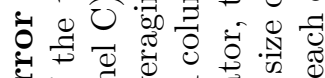

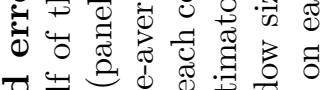

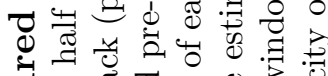
w

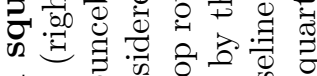

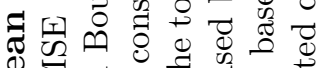
$0 \sum-10000$

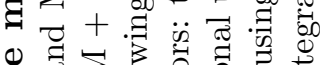

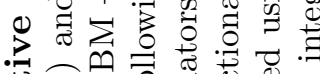

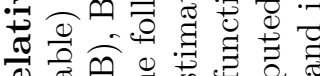

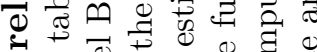
七 สี

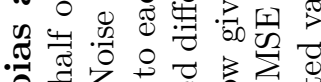
( )

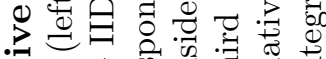
击 +0

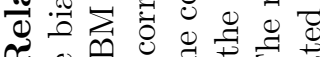

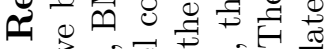

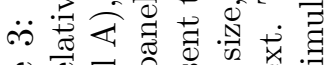

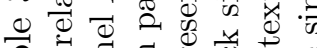

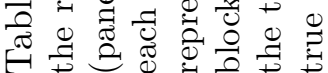

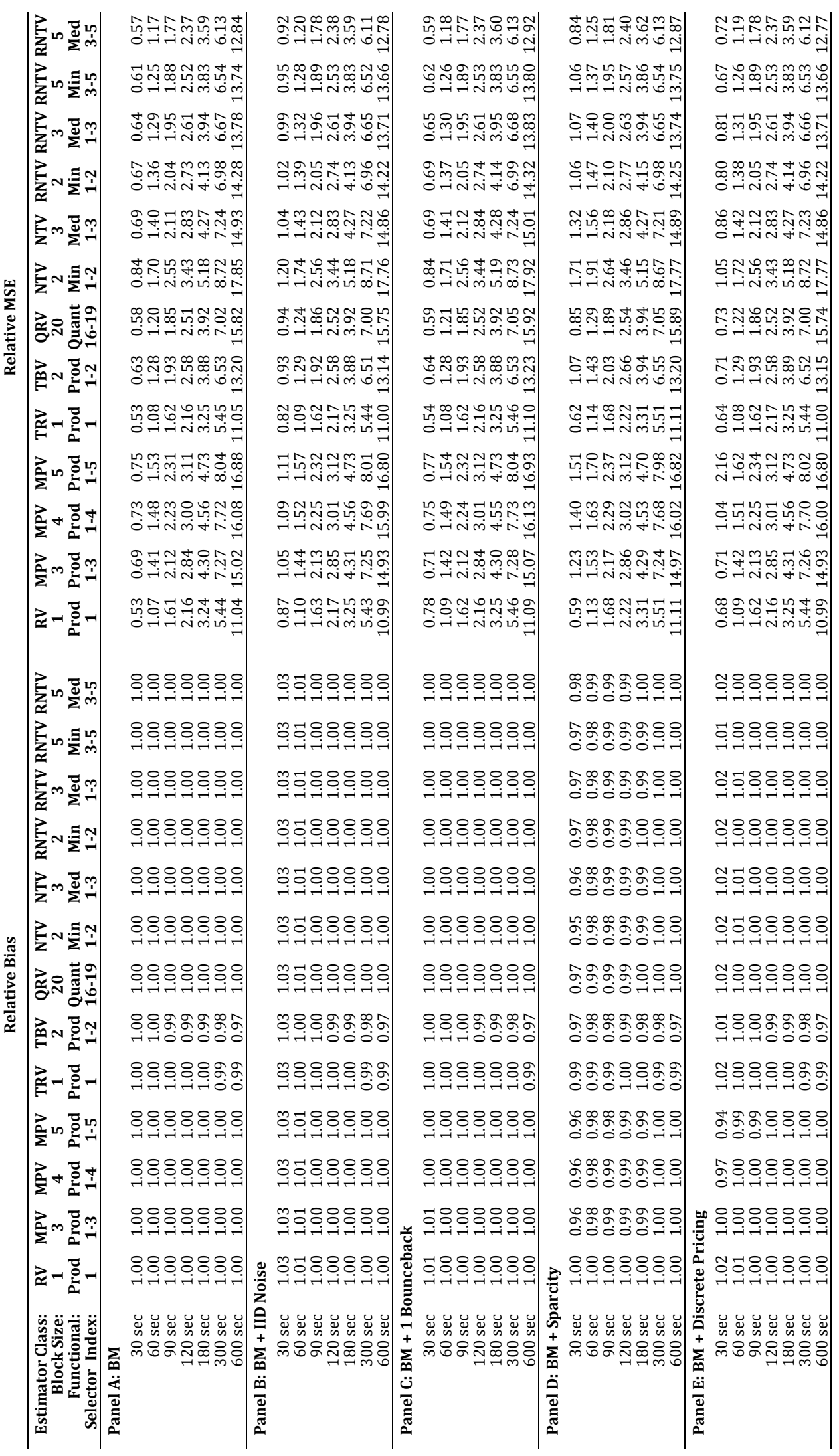



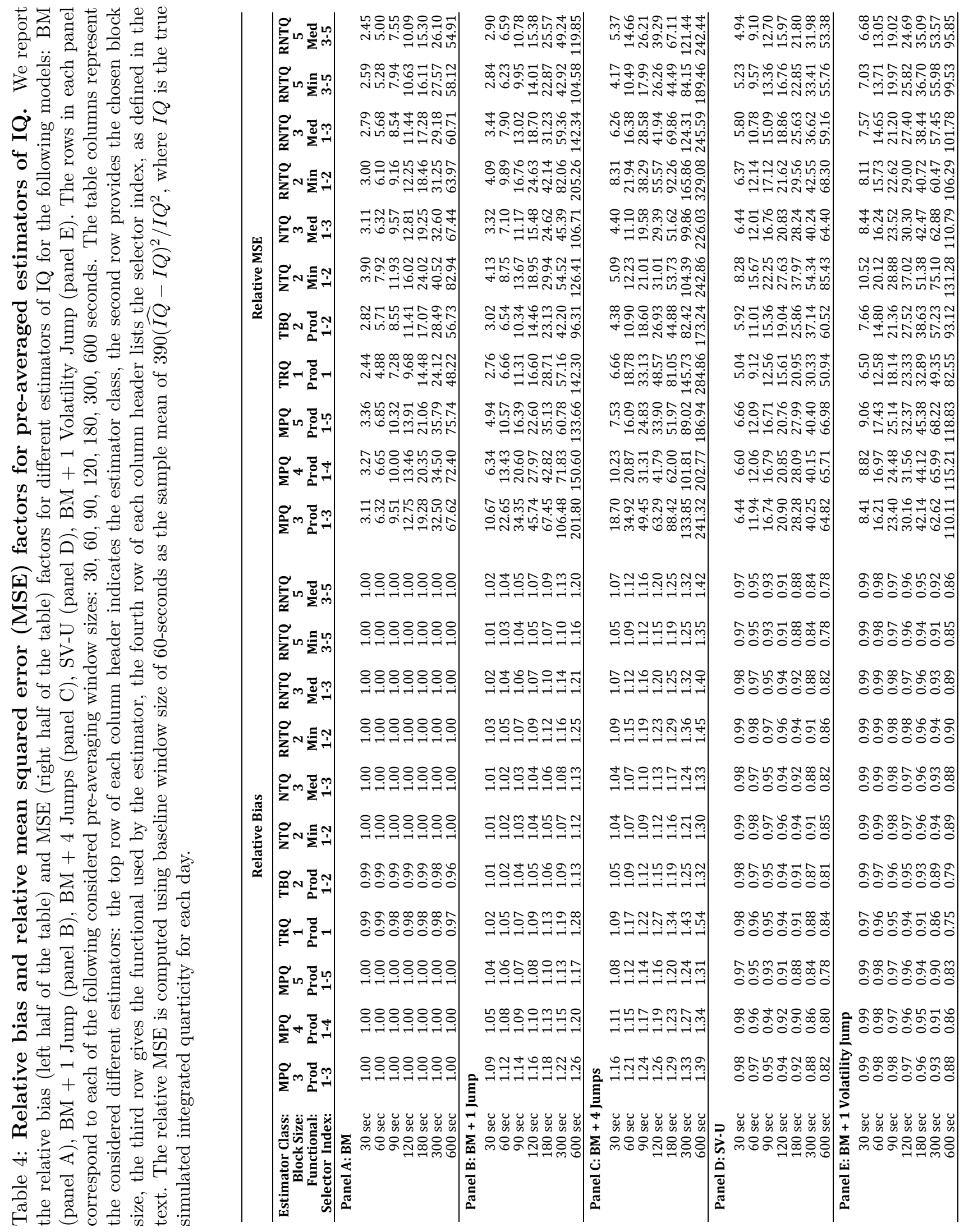

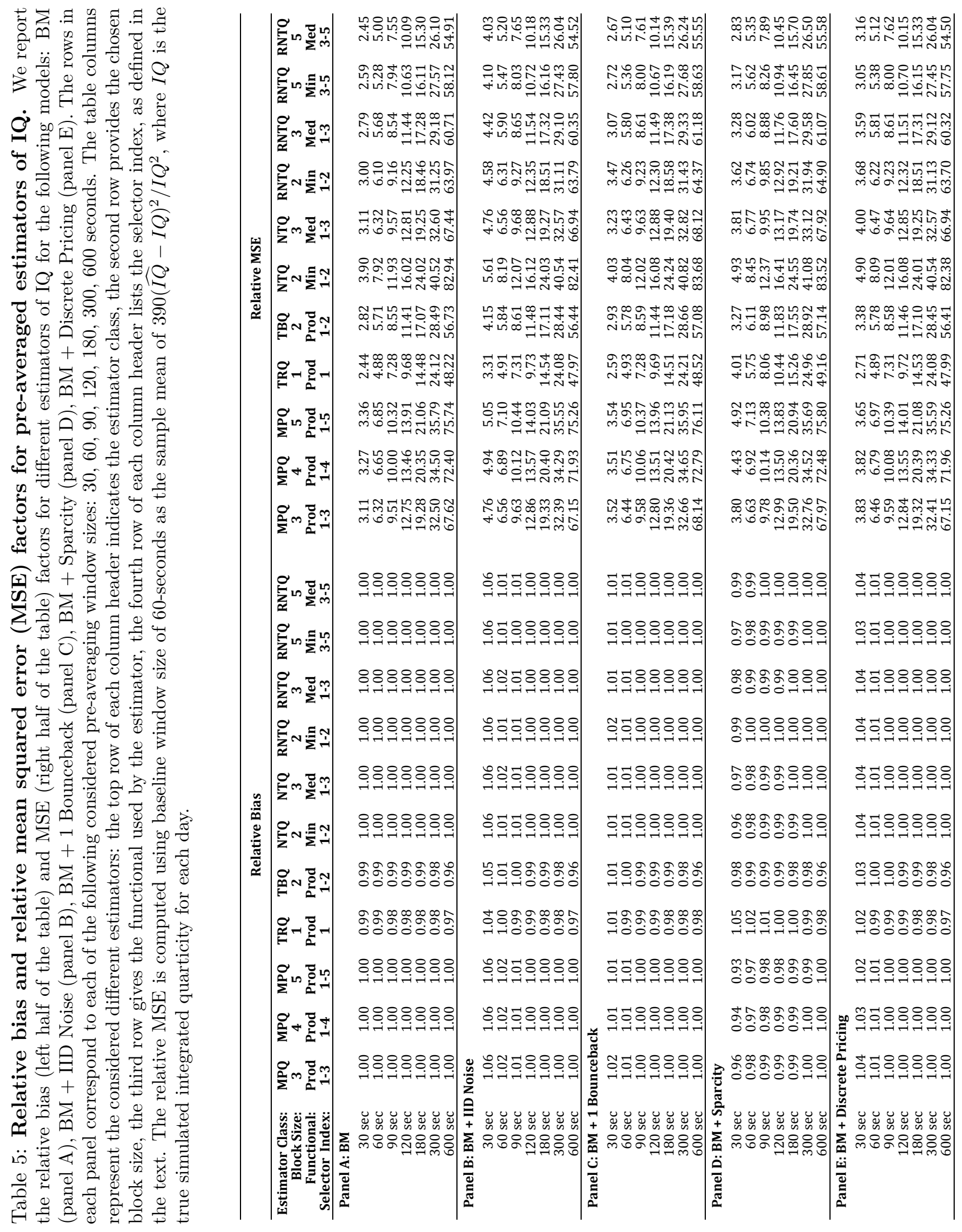


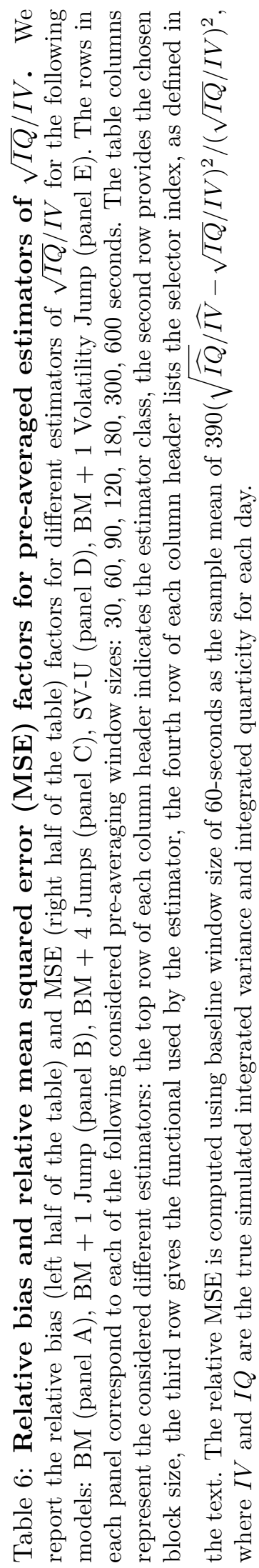

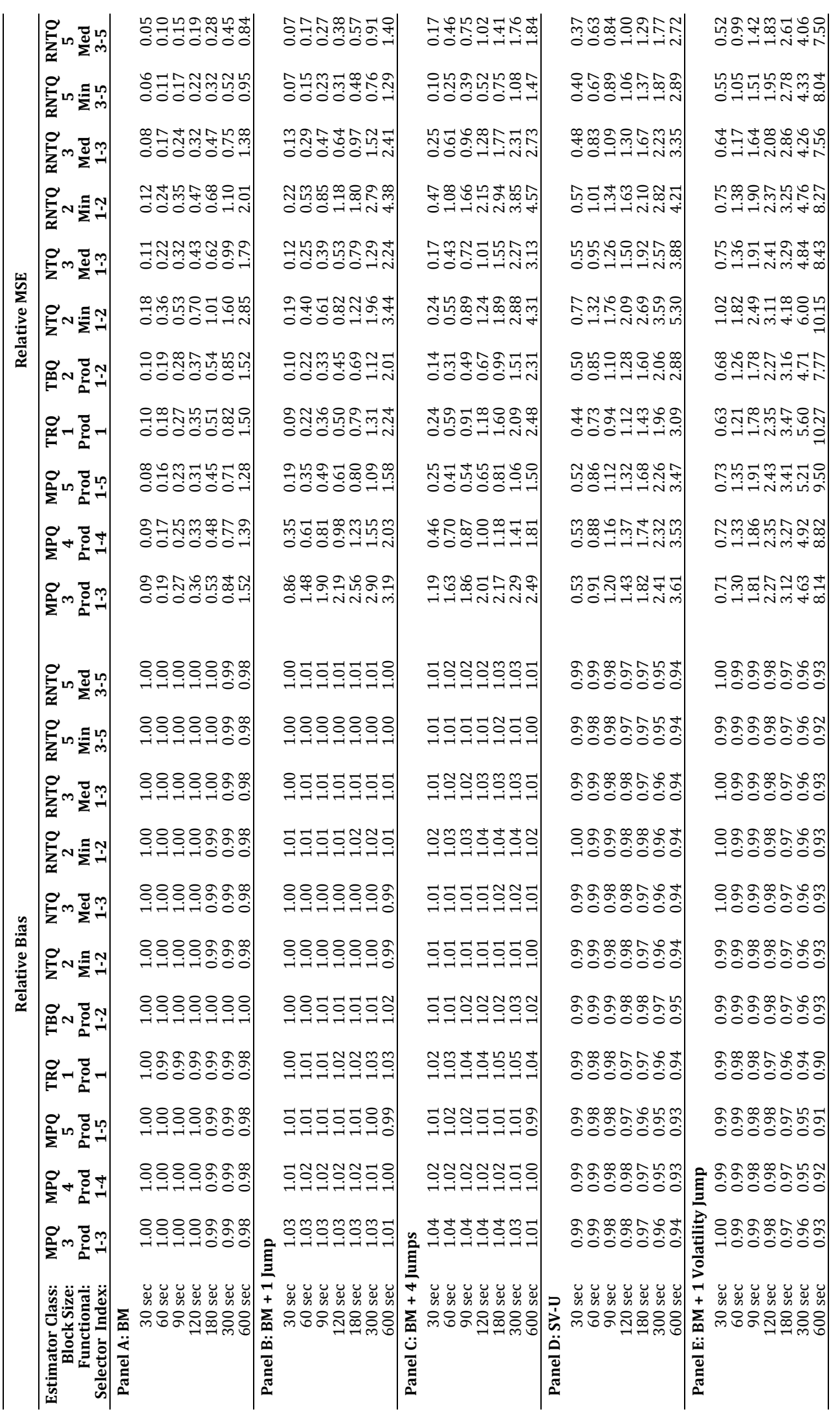




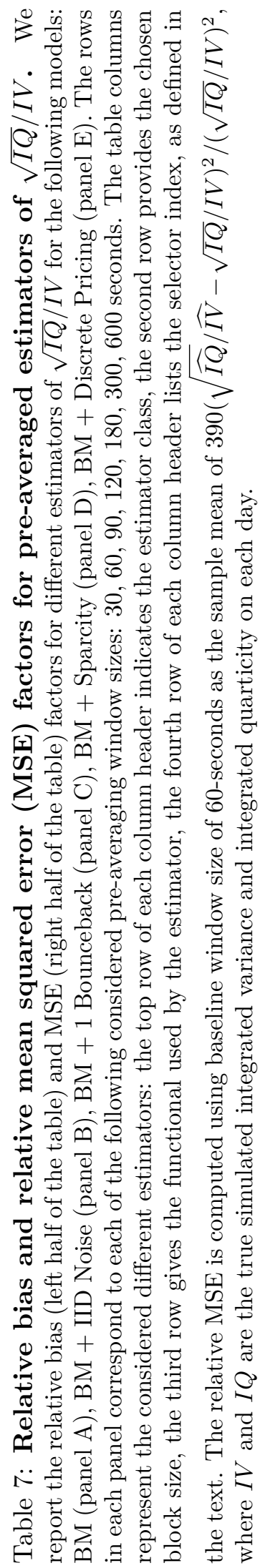

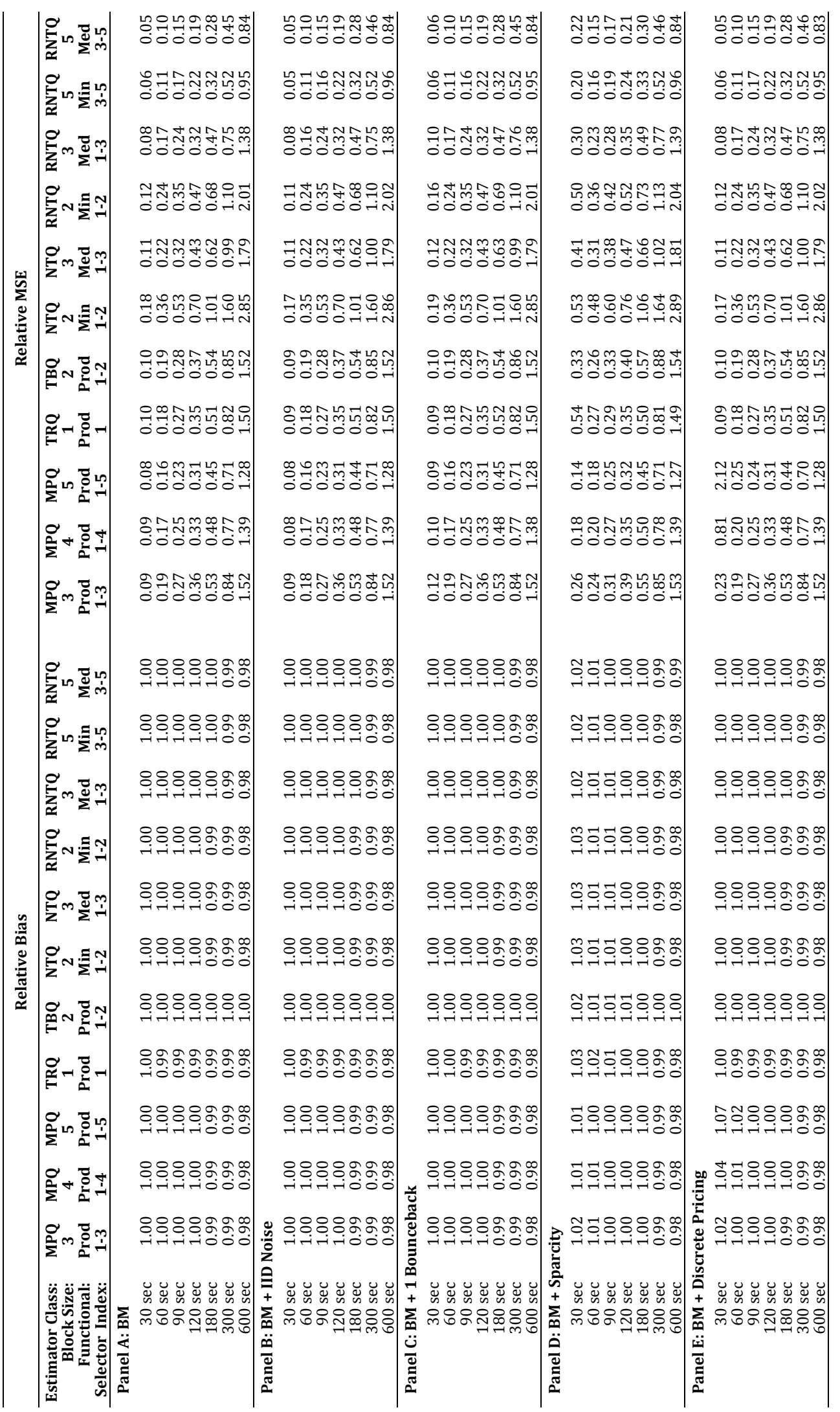




\subsubsection{Jumps in returns: The "BM +1 Jump" and "BM +4 Jump" scenarios}

To compare the finite sample jump-robustness of the estimators, we augment the BM model with return jumps of the Poisson-Gaussian type that are independent of volatility and account for $20 \%$ of the daily QV (25\% of IV). We focus on two scenarios, one with a single jump per day, the "BM +1 Jump" scenario reported in Panel B of Tables 2, 4, and 6, and one with four jumps per day, the "BM +4 Jumps" scenario reported in Panel C of the same tables. Both scenarios feature the same overall jump contribution to daily variance and returns are sampled on an equispaced time grid.

We infer from panels $\mathrm{B}$ and $\mathrm{C}$ in Table 2 that TRV, TBV, QRV, RNTVMin5 and RNTVMed5 provide the best robust IV inference in terms of RMSE. It is also clear that the relative performance of the estimators within this group shifts as we move from a single to four jumps with, in particular, RNTVMin5 and TBV improving their standing as the jump intensity increases. This tendency is even more pronounced for IQ estimation, where panels $\mathrm{B}$ and $\mathrm{C}$ in Table 4 reveal that RNTQMin5 is the best performer in both jump scenarios for frequencies spanning 90 seconds and 3 minutes. Finally, and very strikingly, panels $\mathrm{B}$ and $\mathrm{C}$ of Table 6 show that the pairing of RNTQMin5 and RNTVMin5 dominate all the other estimators by a significant margin in terms of estimating the ratio $\sqrt{I Q} / I V$, which governs the precision of $\log (\mathrm{IV})$. Thus, from the perspective of finite sample jumprobustness, the RNTQ estimators introduced in this paper seem to offer attractive efficiency improvements, especially for estimation of IQ and the ratio $\sqrt{I Q} / I V$.

Juxtaposing Panel B or C in Table 4 and Panel B or C in Table 6, we also note that the distortions induced by jumps appear much less pronounced for the ratio statistic than for IQ. This is due to a partial cancelation of the resulting upward bias in both the numerator and the denominator. Thus, ratio-based inference is likely to be preferable regardless of the choice of estimator. Given the superior performance of the RNTQMin5 and RNTVMin5 pairing, it emerges as a natural candidate for practical jump-robust power variation estimation and jump testing in particular. Below, we explore whether this estimator is robust to other common "irregularities" in high-frequency return data as well.

\subsubsection{Time-varying volatility: The "SV-U" and "BM +1 Volatility Jump" scenarios}

Pronounced intraday variation as well as seemingly abrupt changes (jumps) in spot volatility are prevalent in high-frequency returns. This poses a challenge for power variation estimation, as jump-robust estimators may not be able to distinguish sharply between abrupt shifts in volatility and return jumps. For example, in the context of IV estimation, ADS (2009) provide compelling evidence for a sizeable finite sample distortion in IV estimators when the intraday volatility is stochastic and subject to a diurnal pattern reminiscent of 
the form characterizing most stock data. This section extends their analysis and draws broader conclusions for estimation of IQ and $\sqrt{I Q} / I V$ by exploring two distinct scenarios that violate the (locally) constant volatility assumption.

Our first scenario, denoted "SV-U" (Panel D, Tables 2, 4, and 6), is a modified version of the corresponding model in ADS (2009) where the diurnal pattern now is calibrated to tick-time sampled trade data for individual stocks. In this way, the "SV-U" scenario is roughly tailored to match the deterministic U-Shape pattern in the data used in our empirical illustrations, as shown in Figure $5 .{ }^{21}$ In particular, we simulate a two-factor affine stochastic volatility model and superimpose an asymmetric diurnal pattern (Hasbrouck, 1999) for which the variance at the open is more than four times the midday variance while the variance at the close is about the same as the midday variance. The latter distinguishes the intraday volatility pattern in tick time sampled trade data from tick-time quote data.

The second scenario, denoted "BM + 1 Volatility Jump" (Panel E, Tables 2, 4, and 6 ), involves a six-fold spike in the intraday variance at a point uniformly distributed across the trading day. Volatility is constant before the jump and it remains constant at the new higher level following the jump. In this way, the scenario approximates the effect of sudden bursts in market activity that have inspired the development of alternative volatility jump specifications.

The striking similarity between Panels D and $\mathrm{E}$ of Tables 2, 4, and 6 indicates that these two distinct forms of time variation in volatility have a qualitatively similar impact on the estimators in terms of finite sample bias and RMSE. This may be attributed to the fact that both scenarios render neighboring returns inhomogeneous, resulting in a downward bias due to scaling factors that are incorrectly sized as well as inappropriate truncation of diffusive returns that are misclassified as jumps due to the fluctuating level of the return variance. The less "local" estimators are more exposed to such heterogeneity in returns. This explains the ordering of the biases of the IV and IQ estimators in Panels D and E of tables 2 and 4, with estimators relying on block size one to three being the least biased, those based on blocks of four or five returns being slightly more biased, and finally the estimators relying on substantially larger block sizes (such as 20 for QRV in Table 2) being most biased. In summary, the results concerning the bias in Table 4, Panels D and E, provide compelling evidence against the use of sparser sampling frequencies, such as ten minutes or lower, for IQ estimation. This runs counter to some recommendations in the early literature, suggesting that systematic biases in IQ estimation may be avoided through sparse sampling. In contrast, we find that, regardless of the block size of the IQ estimator, the bias is much more tolerable at sample frequencies higher than two minutes versus ten minutes or lower.

\footnotetext{
${ }^{21}$ Our results are qualitatively similar for the "SV-U" scenario of ADS (2009) which is more consistent with quote data.
} 
Most important of all, Panels D and E in Table 6, confirm that the biases for the ratio $\sqrt{I Q} / I V$ are less pronounced and more uniform across the full range of estimators, as may be expected given that there is scope for partial cancelation of the downward biases affecting both the numerator and denominator. Moreover, the ratio estimator based on RNTQMin5 and RNTVMin5 again performs the best from an efficiency standpoint in spite of its seemingly less desirable block size of five. For comparison, increasing the block size of the MPQ and MPV estimators from three to five enhances rather than reduces the RMSE for $\sqrt{I Q} / I V$ in the "BM +1 Volatility Jump" scenario, as documented in Panel E of Table 6. Consequently, the superior efficiency of RNTQMin5 and RNTVMin5 in estimating the ratio $\sqrt{I Q} / I V$ stems from the design of our RNT estimators rather than from the increased block size.

We conclude that inference based on the ratio $\sqrt{I Q} / I V$ appears to be attractive also under time-varying volatility. Moreover, the RNTQMin5 and RNTVMin5 estimators seem to provide superior performance in this setting as well, particularly for frequencies higher than two minutes.

\subsubsection{Microstructure noise: The "BM + IID Noise", "BM + 1 Bounceback", "BM + Sparcity", and "BM + Discrete Pricing" scenarios}

There is often a delicate tradeoff in the choice of sampling frequency with jump robustness improving and resiliency to market microstructure noise deteriorating as the return interval shrinks. Hence, it is important to analyze the effectiveness in dealing with the adverse impact of various noise features by applying pre-averaging and sampling at moderate frequencies around 1-2 minutes.

Specifically, we consider four separate market imperfections. First, in our "BM + IID Noise" scenario (Panel B, Tables 3, 5, and 7), we simulate Gaussian i.i.d. noise with a noiseto-signal ratio of $\lambda=0.25$, in line with what is typical for trade data on individual stocks. Second, we consider a "BM +1 Bounceback" scenario (Panel C, Tables 3, 5, and 7), in which (isolated) errors in the recorded price induce so-called "bounce-backs" in returns, i.e., two large adjacent jumps of opposite sign due to immediate price reversals. We calibrate the magnitude of the bounceback to match $20 \%$ of the daily QV ( $25 \%$ of IV). The third source of noise is irregular sampling, and the associated results are captured by our "BM + Sparcity" scenario (Panel D, Tables 3, 5, and 7). It is generated via random arrivals of the 7,800 distinct quotes by sampling without replacement from the numbers in the range of 1 through 23,400. While not necessarily realistic, this model is helpful in exploring the potential distortion of the estimators when applied on non-homogeneously sampled returns, effectively inducing spurious variations in their volatility. Finally, in our "BM + Discrete Pricing" scenario (Panel E, Tables 3, 5, and 7), we mimic price decimalization by rounding all intraday prices to the nearest cent with a moderate starting price of $\$ 50$. Price 
discreteness is a major reason for the presence of multiple zero returns in high-frequency samples, leading to pronounced downward biases of many jump-robust estimators. ${ }^{22}$

The first general conclusion to be drawn from Tables 3,5 and 7 is that, irrespective of the particular type of noise, it is necessary to avoid sampling at the highest frequencies (e.g., not exceeding one minute) to obtain reasonably unbiased and efficient estimates of IV, IQ and $\sqrt{I Q} / I V$. Second, the noise reduction benefit from pre-averaging appears to be greatest in the "BM +1 Bounceback" scenario (Panel C, Tables 3, 5, and 7). This is explained by the near perfect cancelation of adjacent jumps of opposite sign when constructing the individual pre-averaged returns (see appendix C). Third and most important, across all noise scenarios, the biases of the IQ estimators (Table 5) are about twice larger than those for the corresponding IV estimators (Table 3), while the biases for the ratio $\sqrt{I Q} / I V$ (Table 7) are smaller. Thus, partial cancelation of biases in numerator and denominator again renders the ratio statistic better behaved in the presence of distortions.

As a final note, there are no dramatic changes in the ordering of the estimators in terms of RMSE in each noise scenario compared to the BM null (Panel A in each table). Our RNTQMin5 and RNTVMin5 estimators retain their appeal, especially for ratio-based inference in the preferred range of frequencies from one to two minutes.

\section{An analysis of the Dow Jones 30 stocks}

This section gauges the empirical performance of competing estimators of $I V, I Q$, and $\sqrt{I Q} / I V$ on the Dow Jones 30 stocks using tick time sampling of NYSE TAQ trade data from January 1, 2005 through July 31, 2009. We split our sample period into a low volatility period, January 2005 - May 2007, and a high volatility period, June 2007 - July 2009. This serves as a robustness check against different noise-to-signal ratios and liquidity levels in the two periods. The main focus is on the estimates for $I Q$ and $\sqrt{I Q} / I V$ while the $I V$ results are provided as a more familiar benchmark for comparison of the RNT estimators and the impact of our proposed filtering scheme with previous studies.

\subsection{Truncation of Return Functionals}

Power variation estimators are generally quite sensitive to deviations from the local diffusive null that may arise due to market microstructure features and sampling errors. While common data-error filtering procedures, e.g., as proposed by BNHLS (2009), do eliminate extreme outliers, they are not sufficient to ensure sensible estimates of IQ on observed stock returns for many of the candidate estimators of interest. The reason IQ (and higher order power variation) estimators are substantially more affected by such irregularities is that IQ

\footnotetext{
${ }^{22} \mathrm{MPV}$ and MPQ, in particular, as they are based on products of adjacent (absolute) returns.
} 
estimators typically are scaled by a factor proportional to the sampling frequency and/or involve raising individual returns to a high power. At high frequencies, even relatively small noise terms can therefore be significantly inflated, leading to dramatic divergence across estimators.

Panel A: Mean of $I Q$ for filtered estimators (truncated local functionals). - = - MPQ3 MPQ5 - - MEDRQ - TRQ = - TBQ RNTQMin5

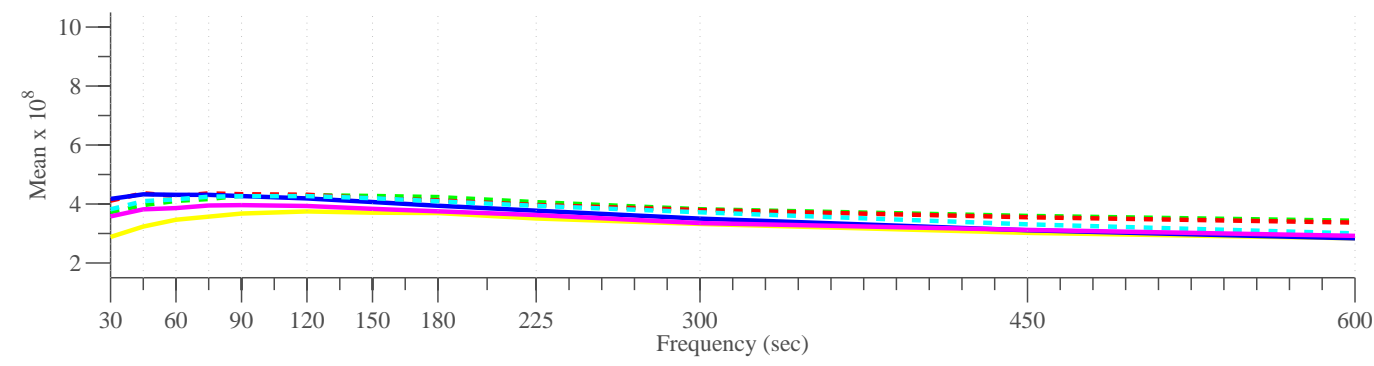

Panel B: Mean of $I Q$ for non-filtered estimators (non-truncated local functionals). = = = MPQ3 $M P Q 5=-=M E D R Q-T R Q==-T B Q-R N T Q M i n 5$

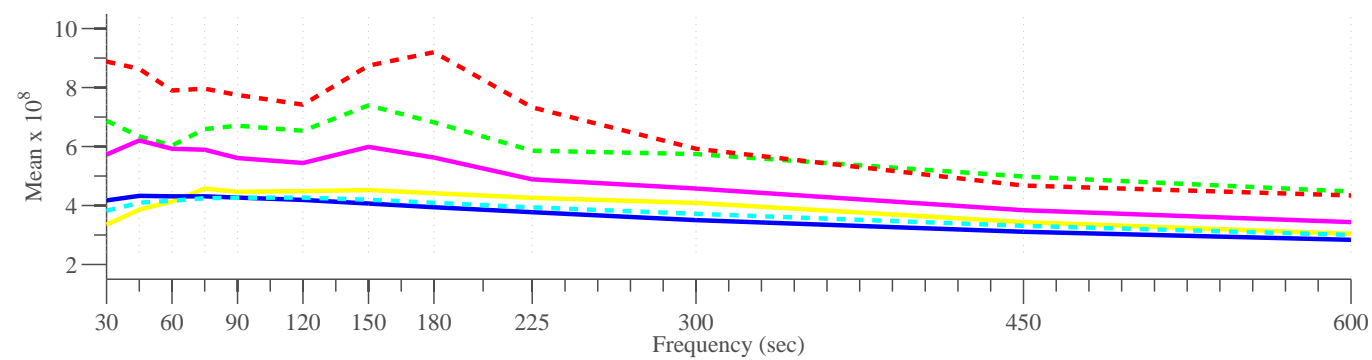

Figure 4: IQ signature plots, showing the effect of filtering via truncation of local functionals (Panel A) versus no-filtering (Panel B) on the average IQ estimate as a function of sampling frequency for subset of estimators. In Panel A all estimators (except TRQ and TBQ) are subject to the novel filtering procedure based on truncation of return functionals as described in Section 2.2, while Panel B depicts their non-filtered counterparts (except TRQ and TBQ). The TRQ and TBQ benchmarks in both panels are based on standard truncation of individual returns. The average is across all stocks in the DJ30 index during the January 2005-May 2007 sampling period and all estimates are pre-averaged and sub-sampled based on tick-time sampling.

Figure 4 illustrates this point by showing that, without additional filtering, most IQ estimators become erratic at higher frequencies. This problem has been noted sporadically in the empirical literature and has motivated some authors to rely on low, and thus relatively inefficient but also less error prone, frequencies for IQ estimation, e.g., BNS (2004a) and Bandi and Russell (2008). Similarly, Jiang and Oomen (2008) uses the squared IV as a simple approximation to IQ, thereby accepting a significant bias in exchange for variance reduction of the IQ estimator. Another striking feature of Figure 4 is that the truncation of return functionals is effective in flattening out the IQ estimators at an average level compatible with the benchmark truncation estimators, TRQ and TBQ. This is encouraging as it suggests that our functional filtering combined with the good efficiency properties of the 
RNT estimators, documented in the simulation section, will render these estimators good candidates for practical applications. Moreover, the nominal size of our filter is set to $10^{-6}$ and the fraction of observations filtered on actual data typically ranges from $0.01 \%$ to $0.05 \%$ for the 60-180 second range, depending on the sampling frequency and the sample period. Overall, more than $70 \%$ of the stock days are untouched by our functional truncation.

It is important to keep in mind the different philosophy behind the mild functional truncation filtering relative to TRQ and TBQ. The latter employ truncation of single returns as the primary tool for achieving jump robustness, and thus need to ensure that asymptotically - all jumps are prevented from impacting the IQ estimator. This requires relatively aggressive truncation and, inevitably, some diffusive returns will also be truncated, motivating the $\mathrm{CPR}$ adjustment to mitigate the resulting finite-sample bias. ${ }^{23} \mathrm{In}$ contrast, the functional filtering is intended only to capture gross deviations from the local Gaussian null hypothesis. As such, the functional truncation will remove some large jumps - along with other data irregularities - inducing truly extreme realizations of the functional, but it will leave many moderately sized jumps untouched. Instead, the impact of the latter is controlled by the functional form of the operator itself, designed explicitly to ensure jump robustness. As may be expected, these fundamentally different rationales behind the truncation procedures induce dramatic differences in the frequencies with which they are applied on real data. For example, the TBQ estimator truncates $1.5 \%-3.0 \%$ of the returns in the 60-180 second range, which is about 100 times more aggressively than for the functional filtering. Likewise, while the functional filtering leaves the returns on the majority of the days in our sample untouched, TBQ truncates at least one observation on $99.9 \%$ of the days. Overall, the evidence is compatible with the objective that the functional filtering serves to control major data irregularities while avoiding excessively intrusive, and potentially distorting, truncation of the underlying returns.

\subsection{The Intraday Volatility Pattern}

It is commonly asserted that an important benefit of tick-time sampling of transactions data is the mitigation of the well-known intra-day volatility U-shape observed in calendar time. Figure 5 demonstrates that the tick-time sampling does succeed in regularizing the average volatility pattern across the main part of the trading day, but there is little impact on the elevated level of volatility in the first 60-90 minutes of trading. Our Monte Carlo experiments found such intraday variation in volatility to be a potent source of systematic and severe biases in power variation estimates, with the ratio statistic $\sqrt{I Q} / I V$ being less sensitive to such distortions than the raw $I Q$ and $I V$ measures.

\footnotetext{
${ }^{23}$ The effects of these procedures were studied explicitly in Section 4.
} 


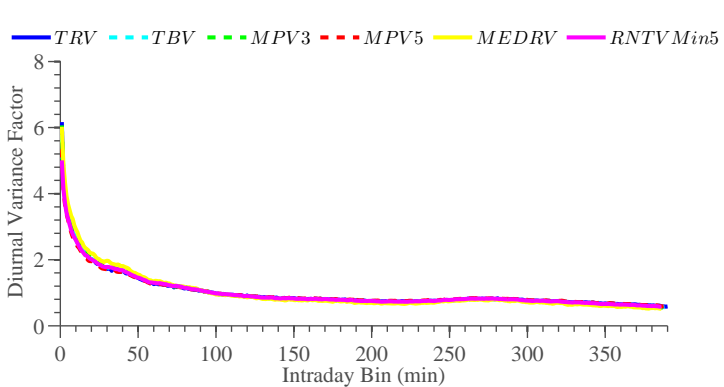

(a)

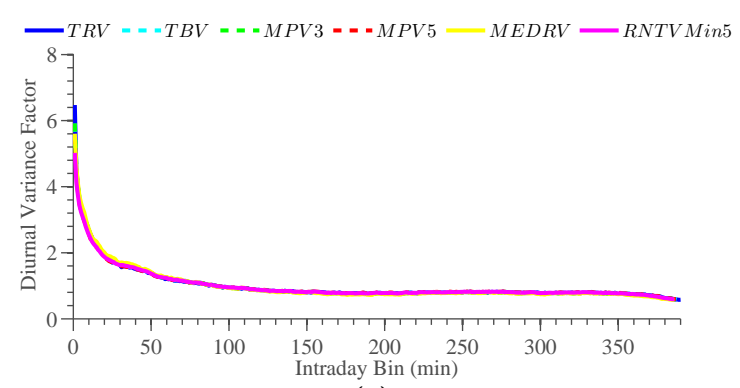

(c)

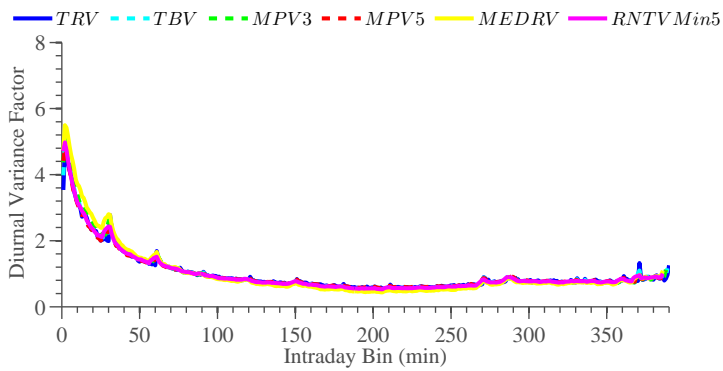

(b)

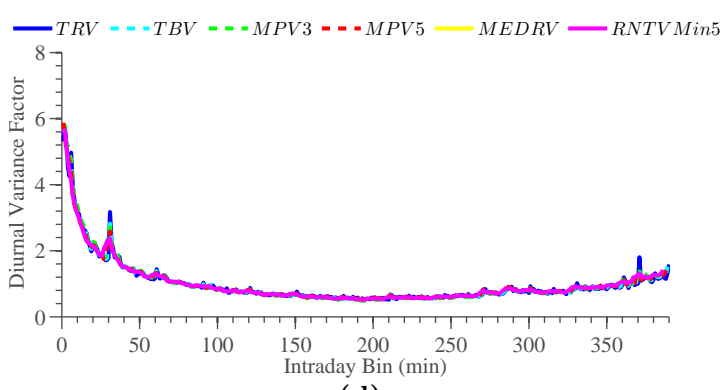

(d)

Figure 5: Diurnal U-shape variance factors across stock-days based on local estimates of $\sigma^{2}$ in one minute buckets using tick time (Panels a and c) or calendar time (Panels b and d) sampling. On each stock-day, the factor in each one-minute bucket is computed by normalizing by the average of the 390 variance estimates on that day. The average variance factor is then computed by averaging across all stock-days in the first sample (Panels a and b) or second sample (Panels c and d).

To systematically assess the empirical relevance of intra-day volatility fluctuations, and in particular the diurnal volatility pattern, for our Dow Jones 30 stock sample, we split the stock-days of each sample period into sub-groups consisting of stock-day combinations representing the top and bottom deciles with respect to a simple scale-free proxy for the daily intraday variation in volatility. This proxy, denoted $V o V$ (volatility-of-volatility), is constructed by splitting the trading day into 26 blocks, and then obtaining the median 30 second absolute returns within each block after first having eliminated all zero returns. Our $V o V$ proxy is then defined as the coefficient of variation (standard deviation divided by mean) of these 26 medians. ${ }^{24}$ Importantly, the $V o V$ measure is designed to pick up any significant variation in intra-day volatility even if it is not in the form of the commonly occurring U-shape. In the following section, we provide signature plots that broadly support our interpretation of the simulation evidence.

\footnotetext{
${ }^{24}$ We find that different constructions of similar robust volatility-of-volatility measures lead to qualitatively similar results in the analysis below.
} 


\subsection{Signature Plots for Integrated Power Variation}

Figures 6 and 7 show IV signature plots for each of the two sample periods. The plots in Panel A represent average estimates across all available stocks and days. They are plotted as a function of the sampling frequency implied by the pre-averaging window size. As expected, the non-jump robust RV estimator lies above the jump-robust IV estimators, which are bundled closely together. ${ }^{25}$ The lone exceptions are MPV5 at frequencies higher than 120 seconds and QRV at frequencies lower than 90 seconds. At the highest frequencies, the drop in MPV5 (and to a lesser extent MPV3) may be explained by the presence of zero returns which has a particularly pronounced impact on these estimators. Consistent with this explanation, RV is the only estimator that does not show any tendency to fall off at the highest frequencies. At the lower frequencies, the striking downward bias of QRV is in line with the evidence gleaned from the simulation results for this particular estimator. While all the jump-robust measures feature negatively sloping signature plots at the lowest frequencies, QRV is easily the one subject to the most significant distortion.

The IQ estimators, shown in Figures 8 and 9, Panel A, display a similar but more pronounced pattern of decline at the lowest sampling frequencies, consistent with the evidence from the simulation scenarios with time-varying volatility. In particular, the estimators are roughly ordered by block size, with the 5-block estimators at the bottom and the 3-block estimators at the top. The truncation based IQ estimators, although nominally based on a short block, are likely disproportionately impacted by time variation in volatility due to the wide window necessary for determining the truncation thresholds, explaining the relatively sharp decline in their signature plots. As mentioned previously, we chose not to include an IQ analogue to the QRV estimator as it, invariably, is more severely downward biased than the others.

The ratio signature plots for $\sqrt{I Q} / I V$ shown in Figures 10-11, Panel A, display a relatively flat but distinct monotonically declining pattern. This reflects the fact that the various microstructure effects, afflicting estimators at the highest frequencies, cancel out more effectively at moderate frequencies consistent with the simulation evidence. The multipower variation estimators display clear abnormalities at the highest frequencies, primarily due to an imperfect offset of the zero returns in the denominator. At sampling frequencies of 60 seconds or lower, however, all the estimators are tightly clustered and downward sloping in accordance with the findings from the simulation scenarios with time-varying volatility.

To further explore the impact of time varying volatility on the estimators in the Dow Jones 30 data, Panels B and $\mathrm{C}$ of each figure depict signature plots, respectively, for the top and bottom deciles with respect to our volatility-of-volatility proxy, $V o V$, across the

\footnotetext{
${ }^{25}$ The distance from the (non-functional filtered) RV curve to the set of robust IV measures in this figure provides an estimate of the average jump contribution to the quadratic return variation across all stocks over the sample period.
} 
combined stock-day sample. It is evident that both the IQ and IV signature plots on high VoV days are dramatically more steeply sloped than on low VoV days, consistent with the hypothesis that the slope is caused primarily by time-varying volatility due to factors like the intraday U-shape pattern, volatility jumps, and volatility bursts associated with news effects. For the ratio statistic (Figures 10-11), the signature plots for low VoV days are essentially (with the exception of the multi-power estimators) flat when viewed on the scale of the high VoV days, highlighting the fact that time variation in volatility also has a pronounced impact on the ratio statistic.

\subsubsection{Panel regressions}

While the totality of the evidence in the preceding sections makes a fairly compelling case that VoV has a pronounced impact on the slope of the signature plots, it is based on grand averages across stocks and days and does not allow for formal statements about statistical significance. To provide a more rigorous analysis, controlling for the potential impact of a few outlier stocks, we run a series of panel regressions, capturing the average effect of VoV on the slope of the signature plot for each individual stock.

Let $\mathcal{E}_{\omega, i, t}$ be any of our IV, IQ or ratio measures estimated at frequency $\omega \in\{30$ sec $, \ldots, 600$ sec $\}$ for stock $i \in\{1, \ldots, 33\}$ on day $t \in\{1, \ldots, N\}$. Choosing $\omega=90$ sec as the baseline, we relate the discrepancy between each specific estimator across the different sampling frequencies to the same estimator at the baseline frequency, $\mathcal{E}_{\omega, i, t}-\mathcal{E}_{90 \text { sec }, i, t}$. A formal measure of the dependence of the slope of the signature plot on $\mathrm{VoV}$, for stock $i$ on day $t$, is then obtained as follows: ${ }^{26}$

$$
\mathcal{E}_{\omega, i, t}-\mathcal{E}_{90 \mathrm{sec}, i, t}=a_{\omega, i}+b_{\omega} \mathrm{VoV}_{i, t}+u_{\omega, i, t}
$$

This panel regression allows for a stock fixed effect but imposes a common slope $b_{\omega}$ across stocks. In Panels A-B of Tables $8-9$ we report the estimated slopes $\hat{b}_{\omega}$ in the first and second sample periods. Based on the evidence from the aggregate IV and IQ signature plots, we expect a negative effect (relative to $90 \mathrm{sec}$ ) of $\mathrm{VoV}$ for frequencies lower than $90 \mathrm{sec}$ and a positive effect for frequencies higher than 90sec. In addition, we might expect this pattern to be somewhat stronger for the "less local" estimators. This prediction is indeed borne out by Panels A-B of Tables 8-9: the impact is clearly monotonic in the sampling frequency $\omega$ and, within a given estimator class, the less local estimators generally display a more significant dependence on VoV. The main exception to this pattern is observed at the 30sec frequency where the results appear less significant, likely due to the confounding effect of microstructure noise. We also note that the level of significance generally is considerably

\footnotetext{
${ }^{26}$ The sample is winsorized as follows: For each sample frequency and estimator, we drop observations less than 2 times the 1 percentile or greater than 2 times the 99 percentile. This procedure eliminates less than $0.5 \%$ of the sample.
} 
lower in the second sample period. This is likely due to the substantially larger number of observations in recent years which, all else equal, tends to reduce the scale of the diurnal volatility pattern for tick-time sampled data.

The panel regression results for the ratio statistic in Panel $\mathrm{C}$ of Tables 8-9 are particularly interesting because the limiting population value of the ratio statistic itself, by construction, is a scale-free measure of volatility of volatility, equal to one if and only if volatility is constant and market microstructure noise is absent. In finite samples, of course, the Jensen effect also induces downwards bias, as documented in our simulation study, and this is further magnified in the presence of time varying volatility. The VoV panel regressions corroborate this fact, revealing the expected monotone pattern at very high levels of significance. Notably, it is not generally true that this effect is stronger for less local estimators, as the bias cancelation between the numerator and denominator again is operative. This is consistent with the estimators being in near full agreement about the average level of the ratio at 60 second and lower frequencies as seen in the signature plots.

In summary, we find all major qualitative features of the simulation designs to also be reflected in the actual data. As such, we deem our recommendations regarding the preferred filtering procedure, choice of estimator and suitable sampling frequency for estimation of IQ and the ratio statistic to be fairly robust. This should allow for improved practical inference regarding IV and the presence of price jumps. 
Figure 6: Average estimates of $I V$ across 33 DJ30 stocks between January 1, 2005 and May 31, 2007. We provide signature plots for the mean of each pre-averaged estimator of $I V$ as a function of pre-averaging window size matching the sampling frequency (measured in seconds on the x-axis). Panel (A) plots the mean across all days. Panel (B) plots the mean across the top $10 \%$ days with respect to intraday variation in volatility. Panel (C) plots the mean across the bottom $10 \%$ days with respect to intraday variation in volatility. Intraday variation in volatility is measured by the $V o V$ measure of volatility of volatility described in Section 5 .

Panel A: Mean of $I V$ for all days.

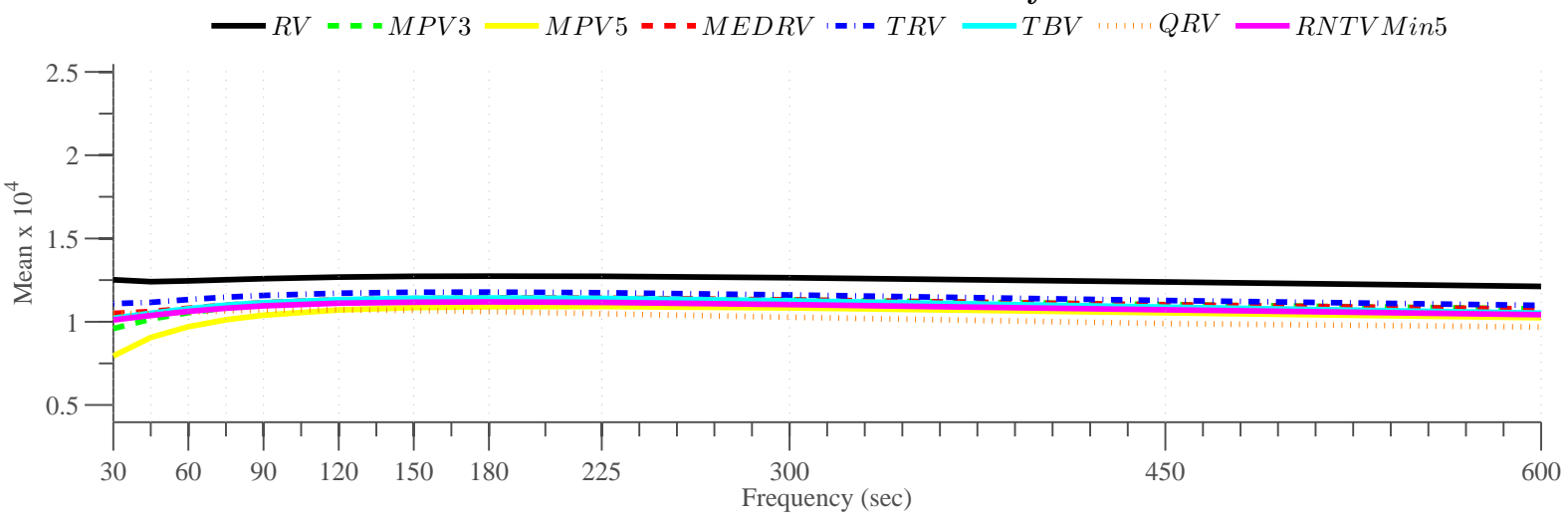

Panel B: Mean of $I V$ for the top $\mathbf{1 0 \%}$ days in terms of intraday variation of volatility. - $R V=-=M P V 3=M P V 5=-M E D R V=T R V=T B V \ldots \ldots R V=R N T V M i n 5$

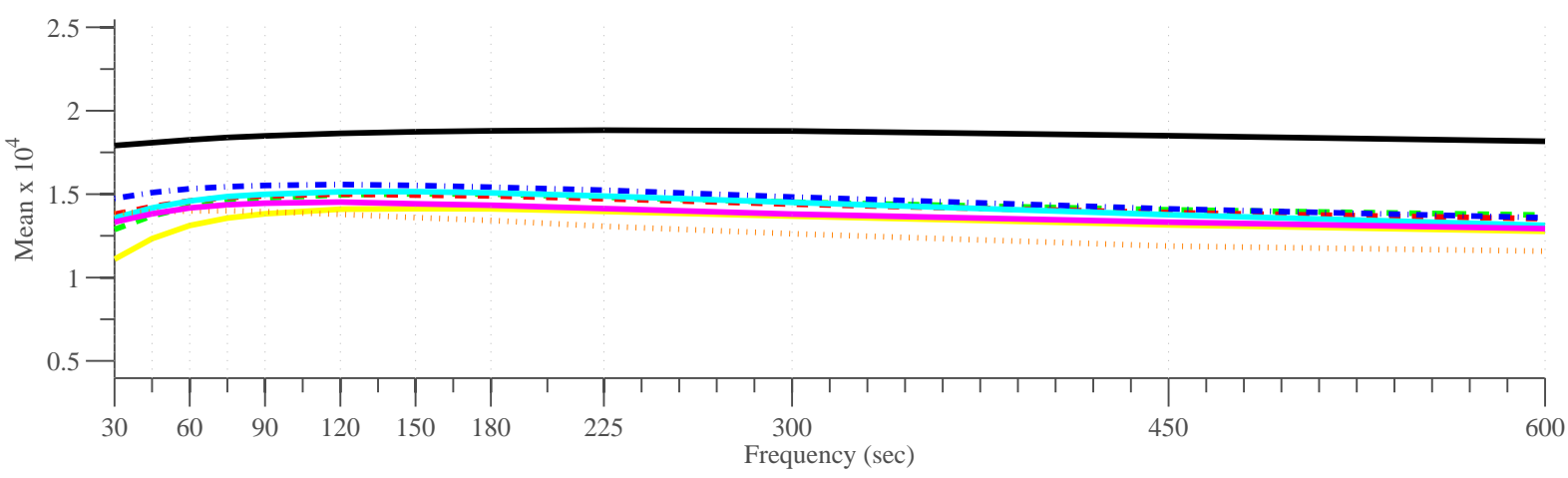

Panel C: Mean of $I V$ for the bottom $10 \%$ days in terms of intraday variation of volatility. - $R V==M P V 3=M P V 5=-M E D R V=T R V=T B V \cdots \cdots Q R V=R N T V M i n 5$

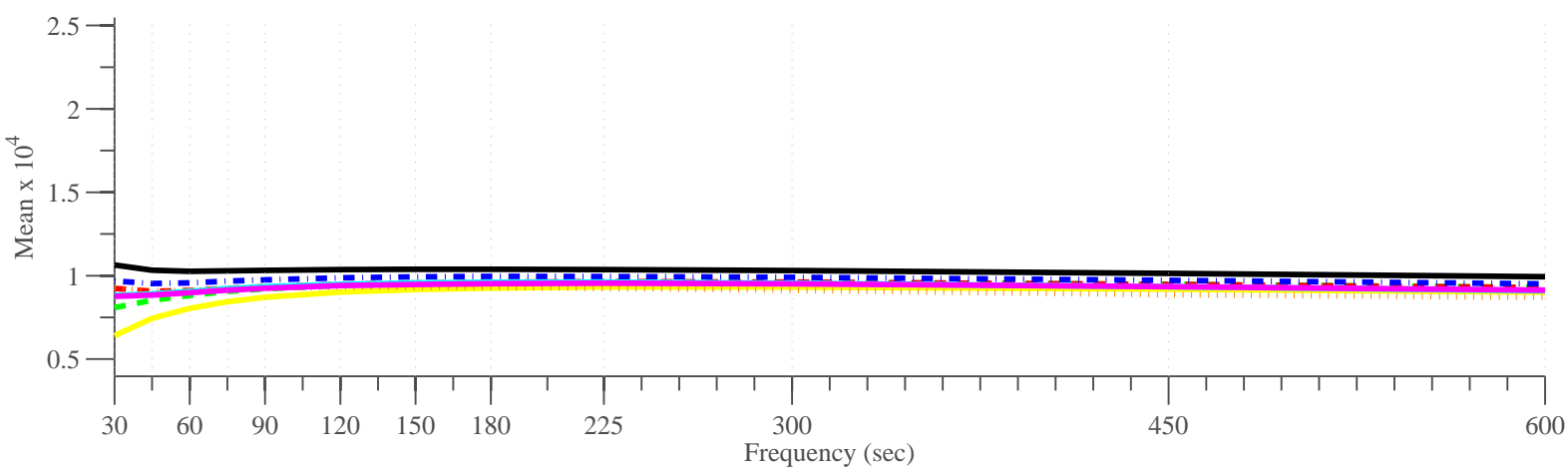


Figure 7: Average estimates of $I V$ across 33 DJ30 stocks between June 1, 2007 and July 31, 2009. We provide signature plots for the mean of each pre-averaged estimator of $I V$ as a function of pre-averaging window size matching the sampling frequency (measured in seconds on the x-axis). Panel (A) plots the mean across all days. Panel (B) plots the mean across the top $10 \%$ days with respect to intraday variation in volatility. Panel (C) plots the mean across the bottom $10 \%$ days with respect to intraday variation in volatility. Intraday variation in volatility is measured by the $V o V$ measure of volatility of volatility described in Section 5 .

Panel A: Mean of $I V$ for all days.

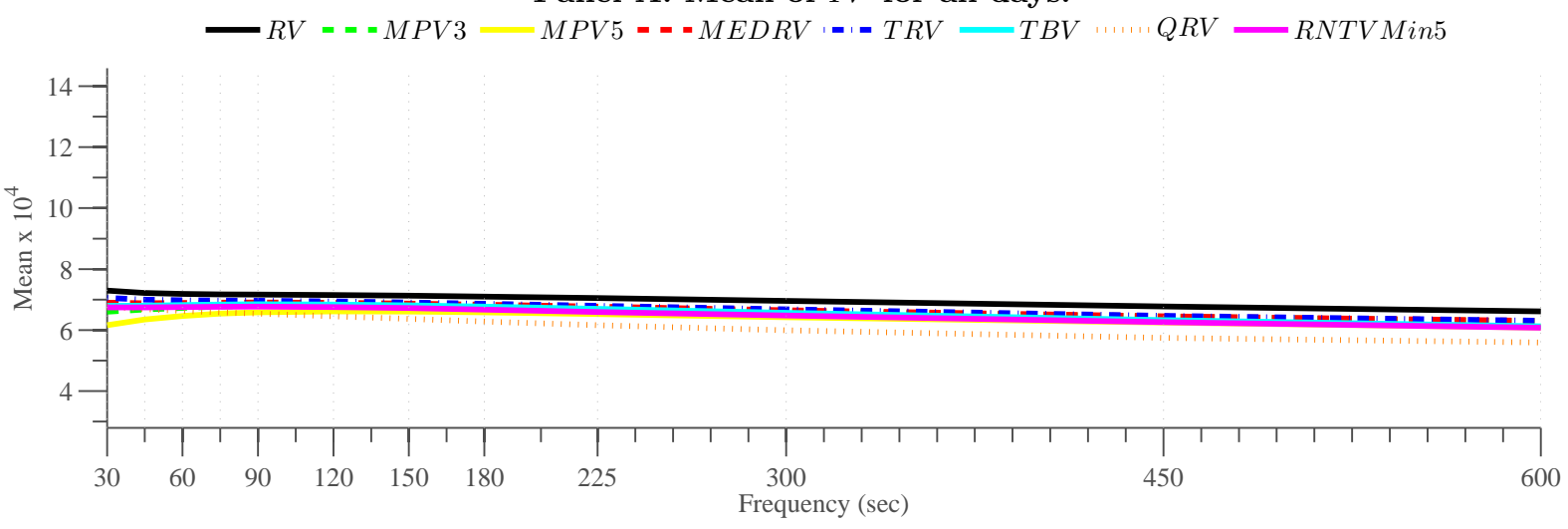

Panel B: Mean of $I V$ for the top $10 \%$ days in terms of intraday variation of volatility. - $R V==M P V 3=M P V 5=-M E D R V=T R V=T B V \cdots \cdots Q R V=R N T V M i n 5$

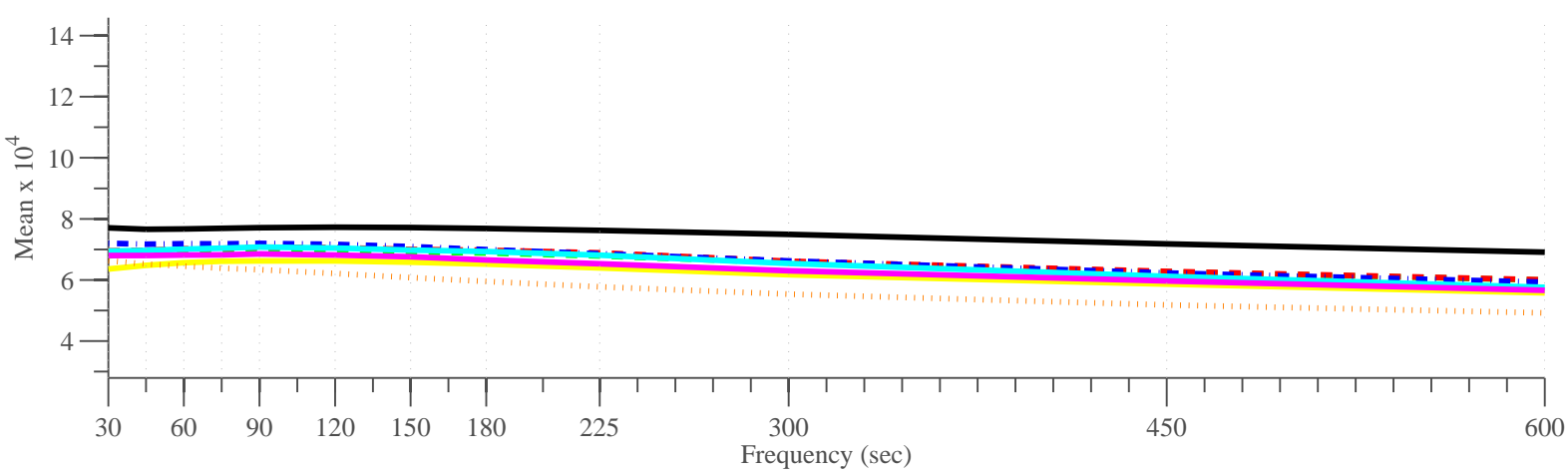

Panel C: Mean of $I V$ for the bottom $10 \%$ days in terms of intraday variation of volatility. $-R V=-M P V 3-M P V 5=-M E D R V=T R V=T B V \ldots \ldots R V-R N T V M i n 5$

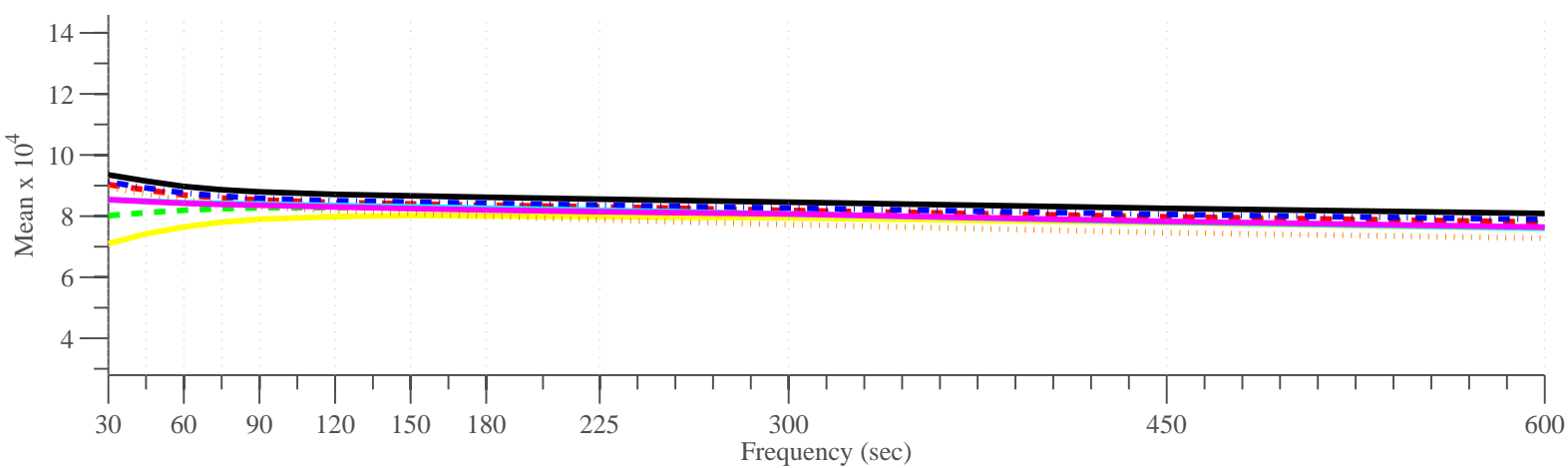


Figure 8: Average estimates of $\sqrt{I Q}$ across 33 DJ30 stocks between January 1, 2005 and May 31, 2007. We provide signature plots for the mean of each pre-averaged estimator of $\sqrt{I Q}$ as a function of preaveraging window size matching the sampling frequency (measured in seconds on the x-axis). Panel (A) plots the mean across all days. Panel (B) plots the mean across the top $10 \%$ days with respect to intraday variation in volatility. Panel (C) plots the mean across the bottom $10 \%$ days with respect to intraday variation in volatility. Intraday variation in volatility is measured by the $V o V$ measure of volatility of volatility described in Section 5 .

Panel A: Mean of $\sqrt{I Q}$ for all days.

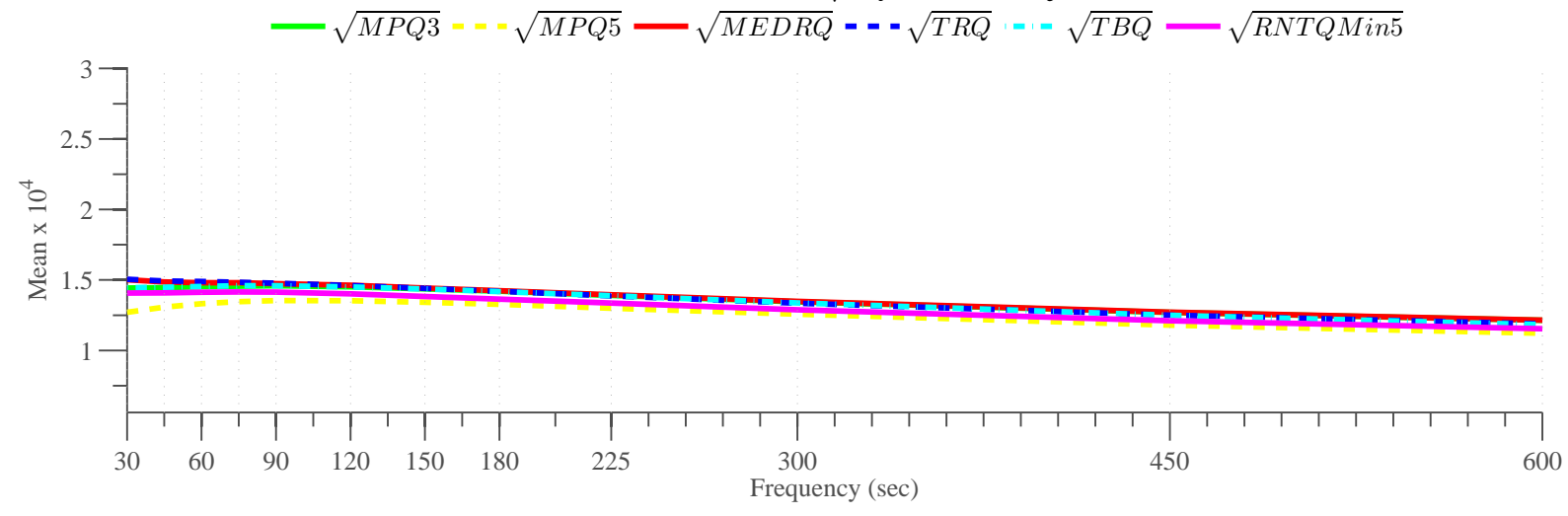

Panel B: Mean of $\sqrt{I Q}$ for the top $10 \%$ days in terms of intraday variation of volatility.

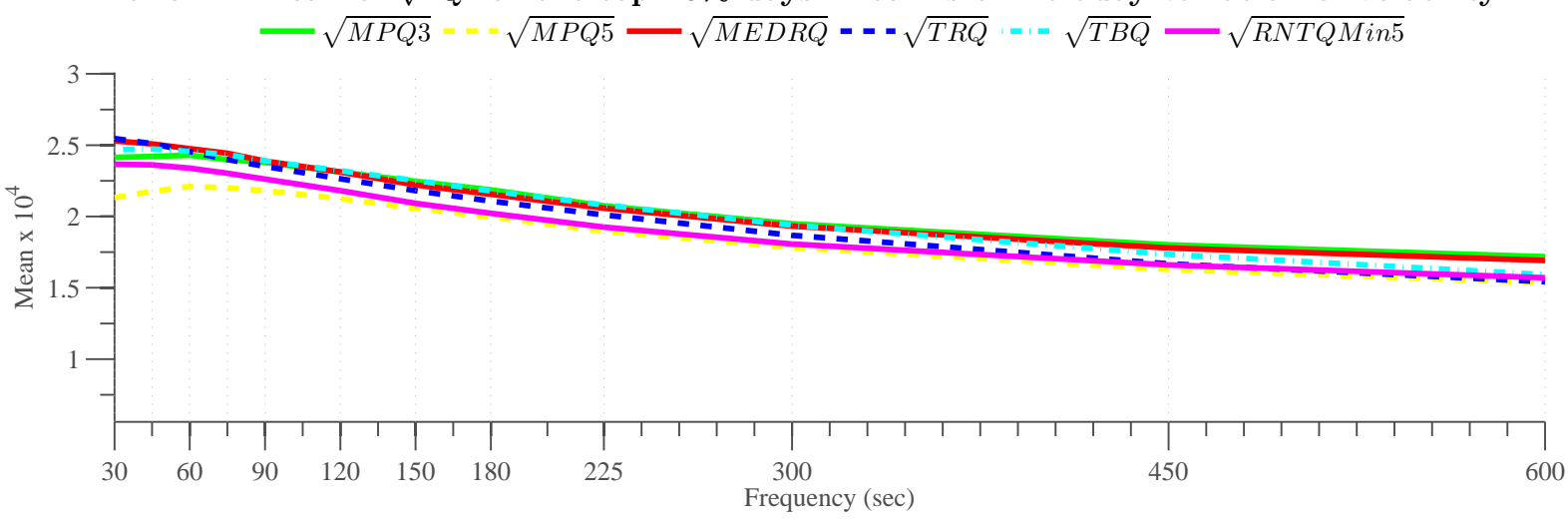

Panel C: Mean of $\sqrt{I Q}$ for the bottom $10 \%$ days in terms of intraday variation of volatility.

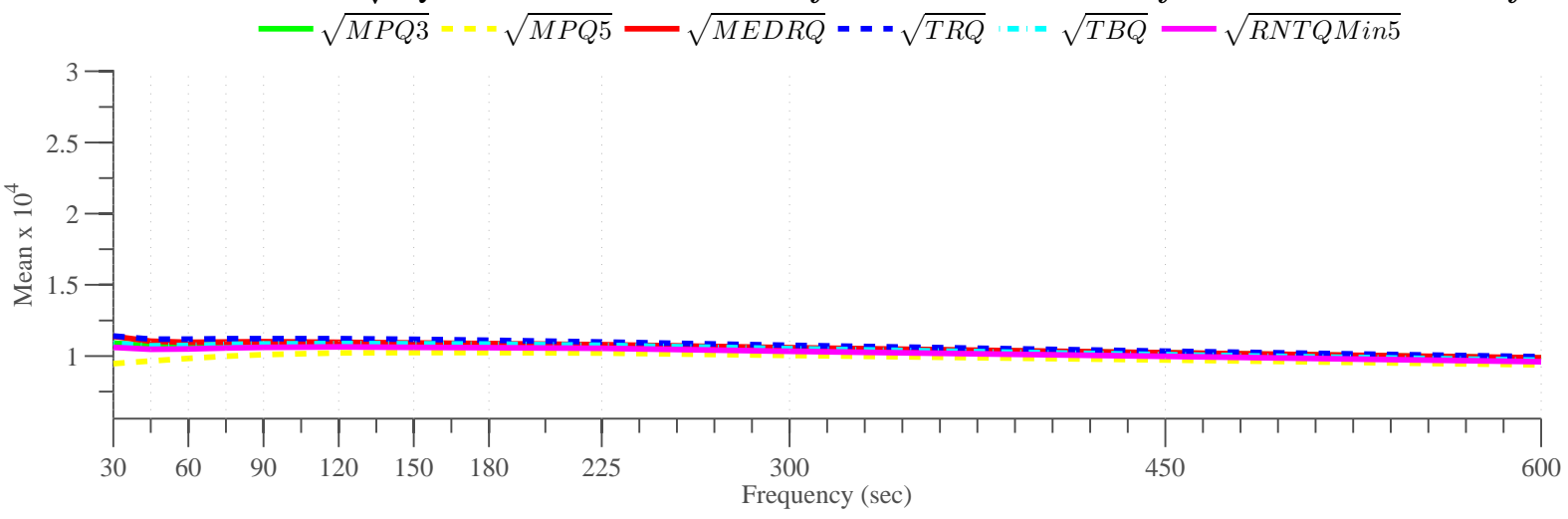


Figure 9: Average estimates of $\sqrt{I Q}$ across 33 DJ30 stocks between June 1, 2007 and July 31, 2009. We provide signature plots for the mean of each pre-averaged estimator of $\sqrt{I Q}$ as a function of pre-averaging window size matching the sampling frequency (measured in seconds on the x-axis). Panel (A) plots the mean across all days. Panel (B) plots the mean across the top $10 \%$ days with respect to intraday variation in volatility. Panel (C) plots the mean across the bottom $10 \%$ days with respect to intraday variation in volatility. Intraday variation in volatility is measured by the $V o V$ measure of volatility of volatility described in Section 5 .

Panel A: Mean of $\sqrt{I Q}$ for all days.

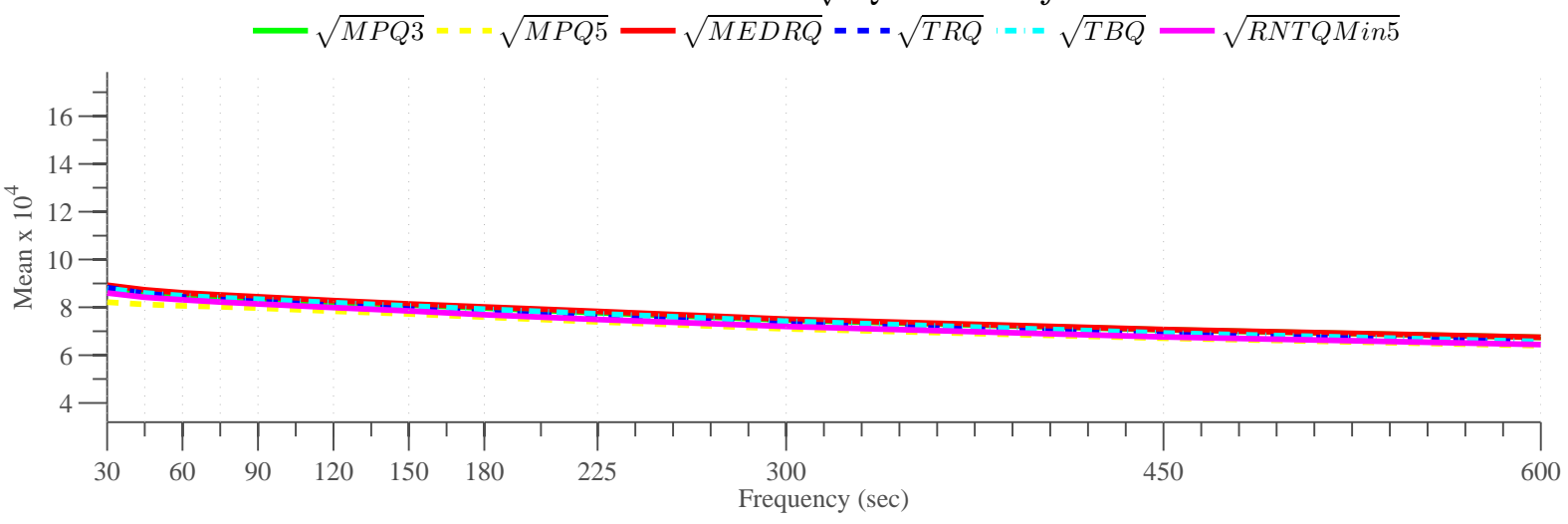

Panel B: Mean of $\sqrt{I Q}$ for the top $10 \%$ days in terms of intraday variation of volatility. $-\sqrt{M P Q 3}=-\sqrt{M P Q 5}-\sqrt{M E D R Q}=-\sqrt{T R Q}=-\sqrt{T B Q}-\sqrt{R N T Q M i n 5}$

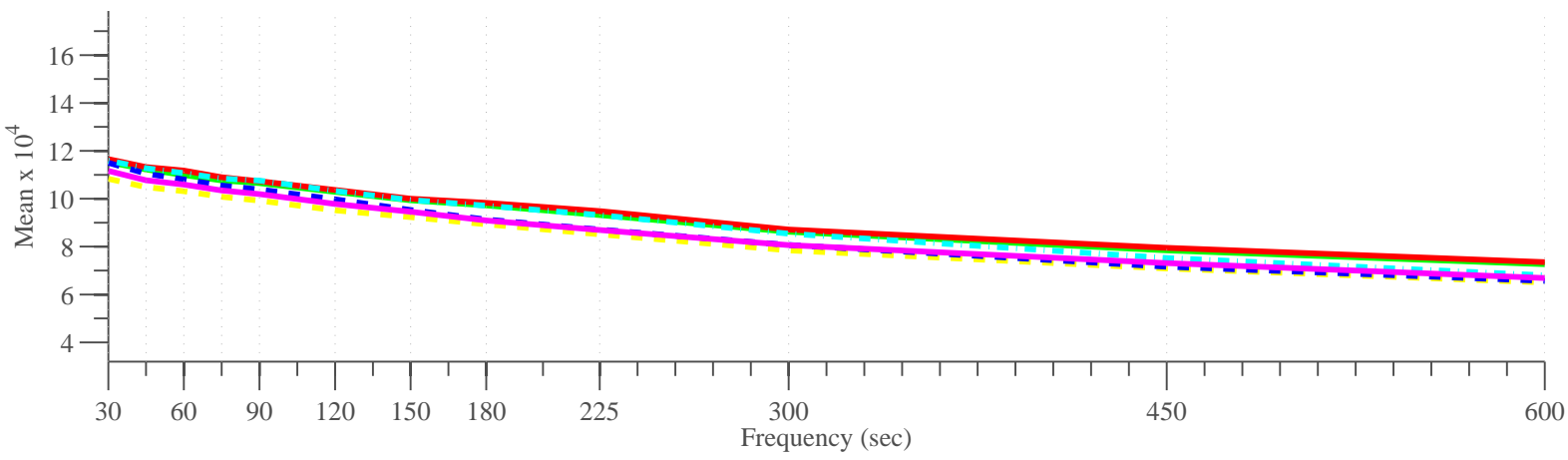

Panel C: Mean of $\sqrt{I Q}$ for the bottom $10 \%$ days in terms of intraday variation of volatility.

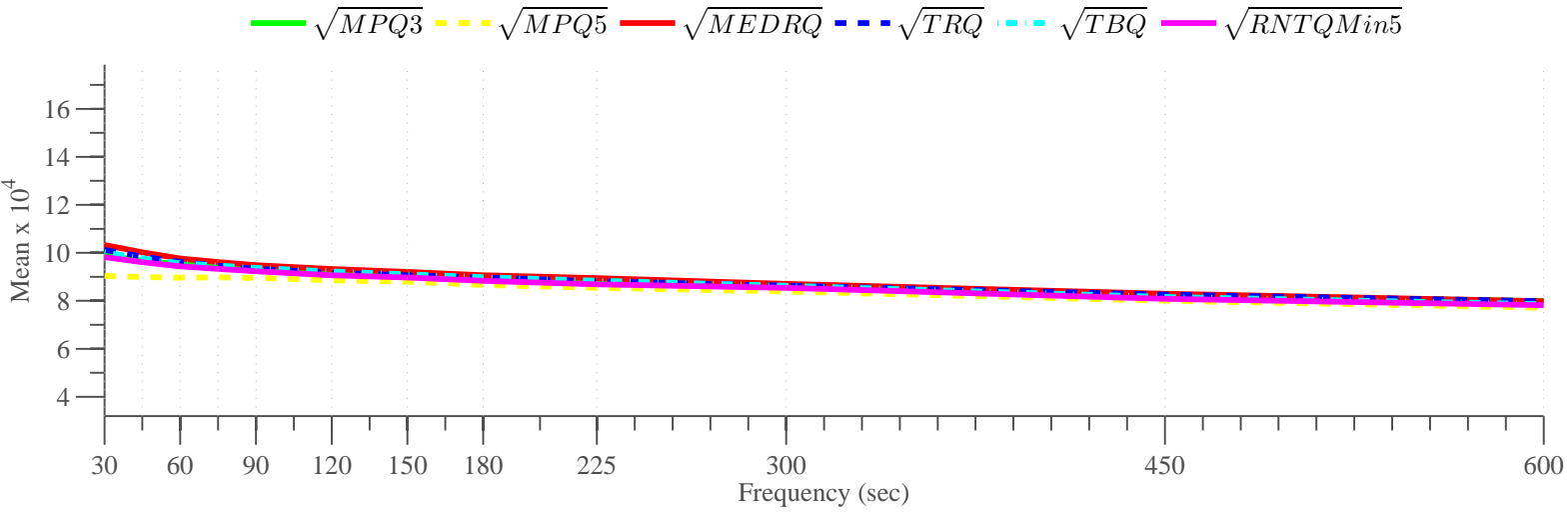


Figure 10: Average estimates of $\sqrt{I Q} / I V$ across 33 DJ30 stocks between January 1, 2005 and May 31, 2007. We provide signature plots for the mean of each pre-averaged estimator of $\sqrt{I Q} / I V$ as a function of pre-averaging window size matching the sampling frequency (measured in seconds on the $\mathrm{x}$-axis). Panel (A) plots the mean across all days. Panel (B) plots the mean across the top 10\% days with respect to intraday variation in volatility. Panel (C) plots the mean across the bottom $10 \%$ days with respect to intraday variation in volatility. Intraday variation in volatility is measured by the $V o V$ measure of volatility of volatility described in Section 5 .

Panel A: Mean of $\sqrt{I Q} / I V$ for all days.

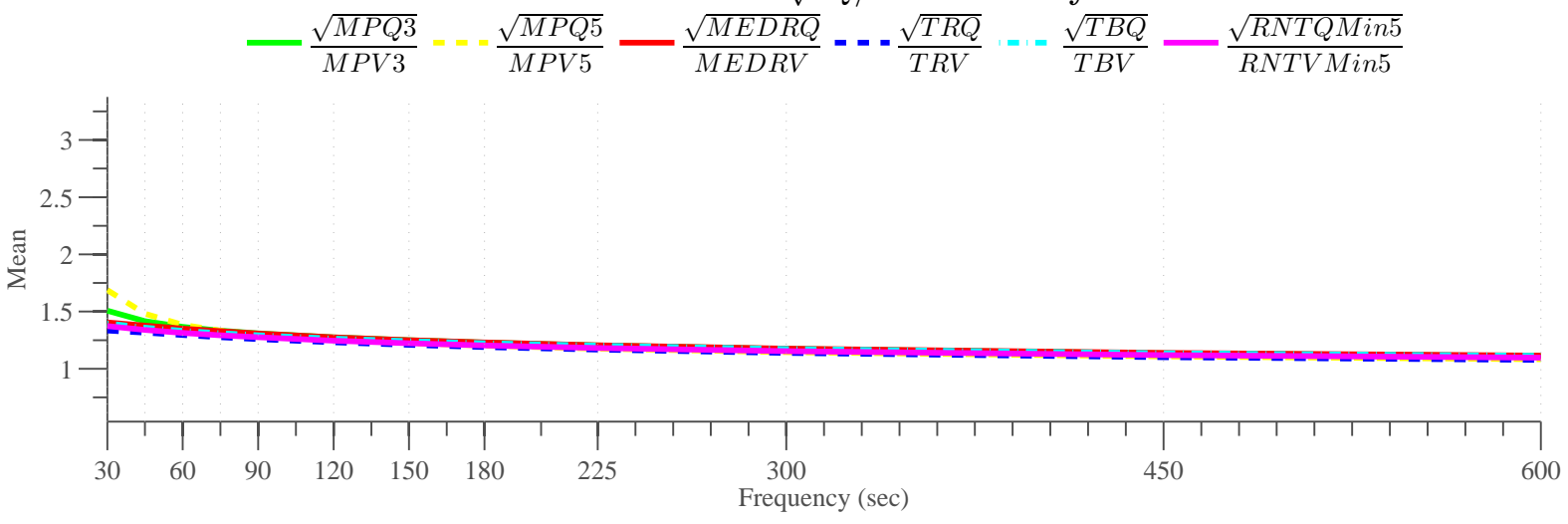

Panel B: Mean of $\sqrt{I Q} / I V$ for the top $10 \%$ days in terms of intraday variation of volatility.

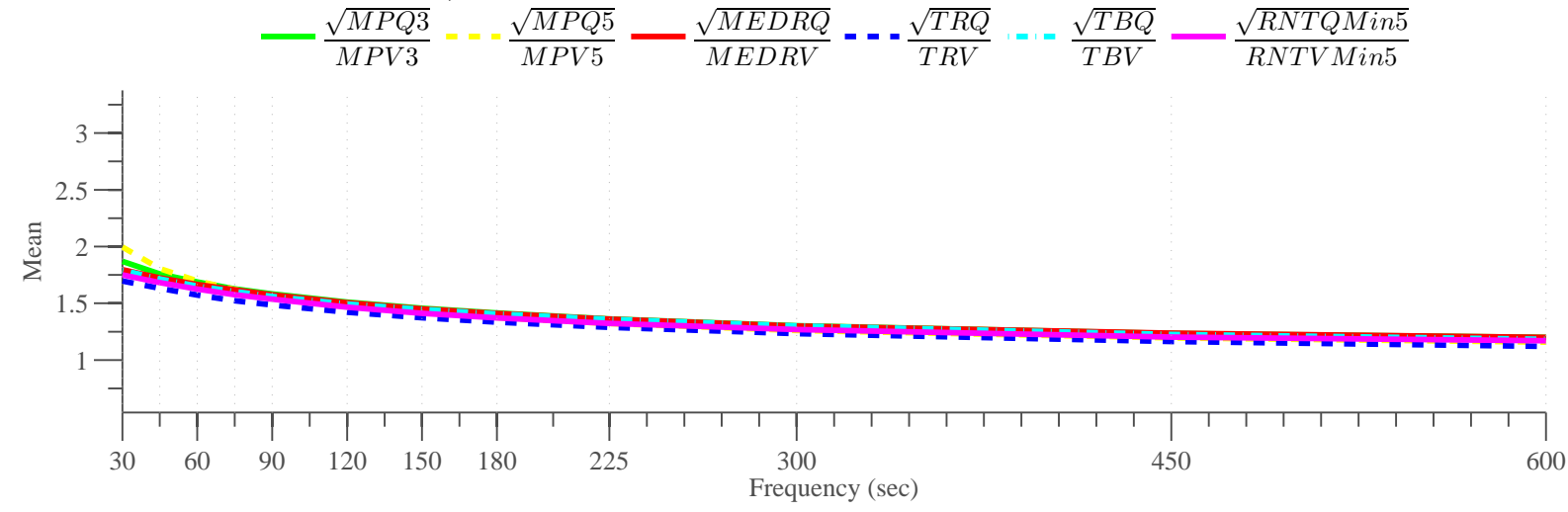

Panel C: Mean of $\sqrt{I Q} / I V$ for the bottom $10 \%$ days in terms of intraday variation of volatility.

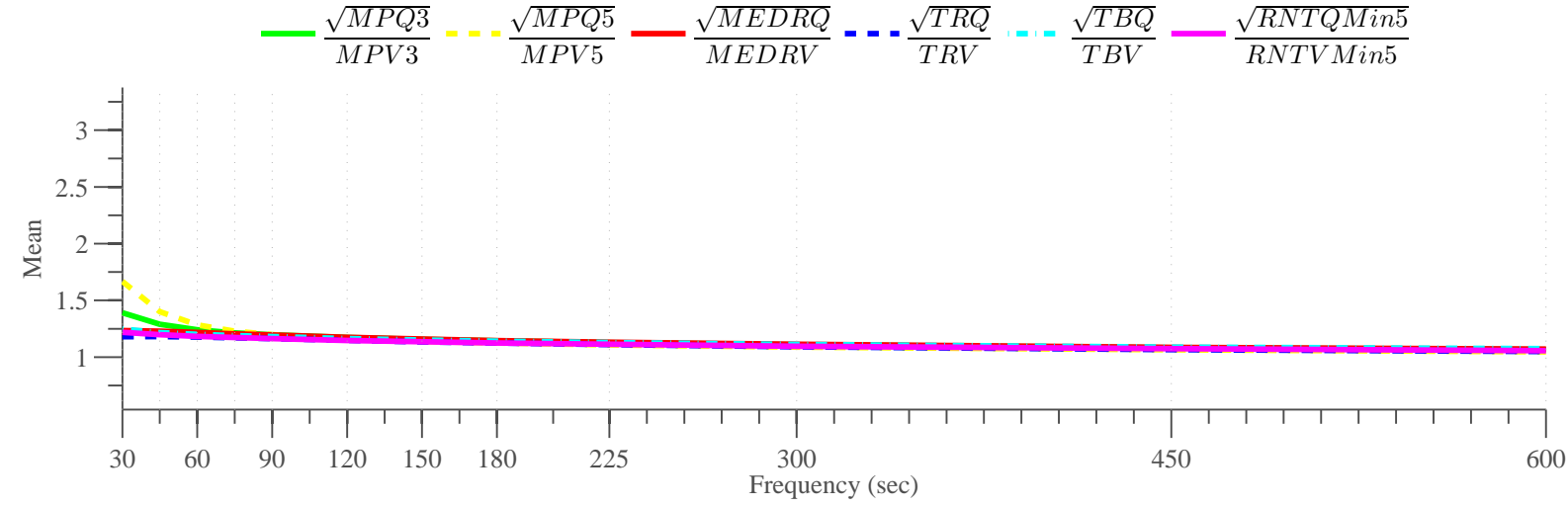


Figure 11: Average estimates of $\sqrt{I Q} / I V$ across 33 DJ30 stocks between June 1, 2007 and July 31, 2009. We provide signature plots for the mean of each pre-averaged estimator of $\sqrt{I Q} / I V$ as a function of pre-averaging window size matching the sampling frequency (measured in seconds on the x-axis). Panel (A) plots the mean across all days. Panel (B) plots the mean across the top $10 \%$ days with respect to intraday variation in volatility. Panel (C) plots the mean across the bottom $10 \%$ days with respect to intraday variation in volatility. Intraday variation in volatility is measured by the $V o V$ measure of volatility of volatility described in Section 5 .

Panel A: Mean of $\sqrt{I Q} / I V$ for all days.

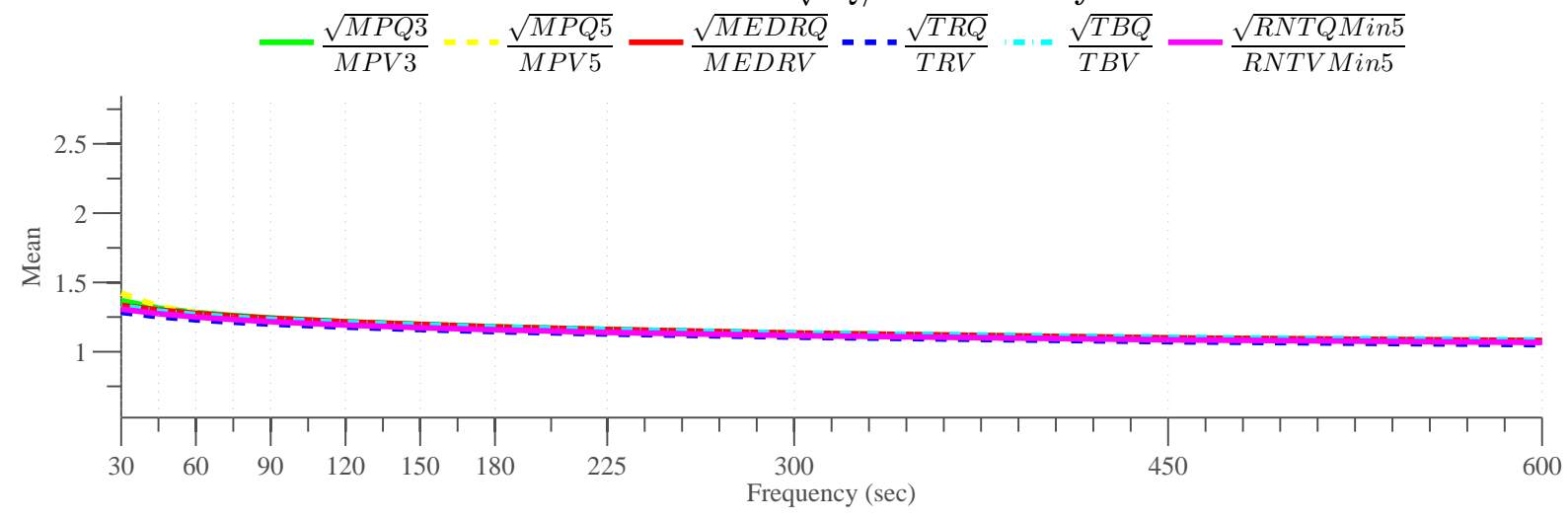

Panel B: Mean of $\sqrt{I Q} / I V$ for the top $10 \%$ days in terms of intraday variation of volatility.

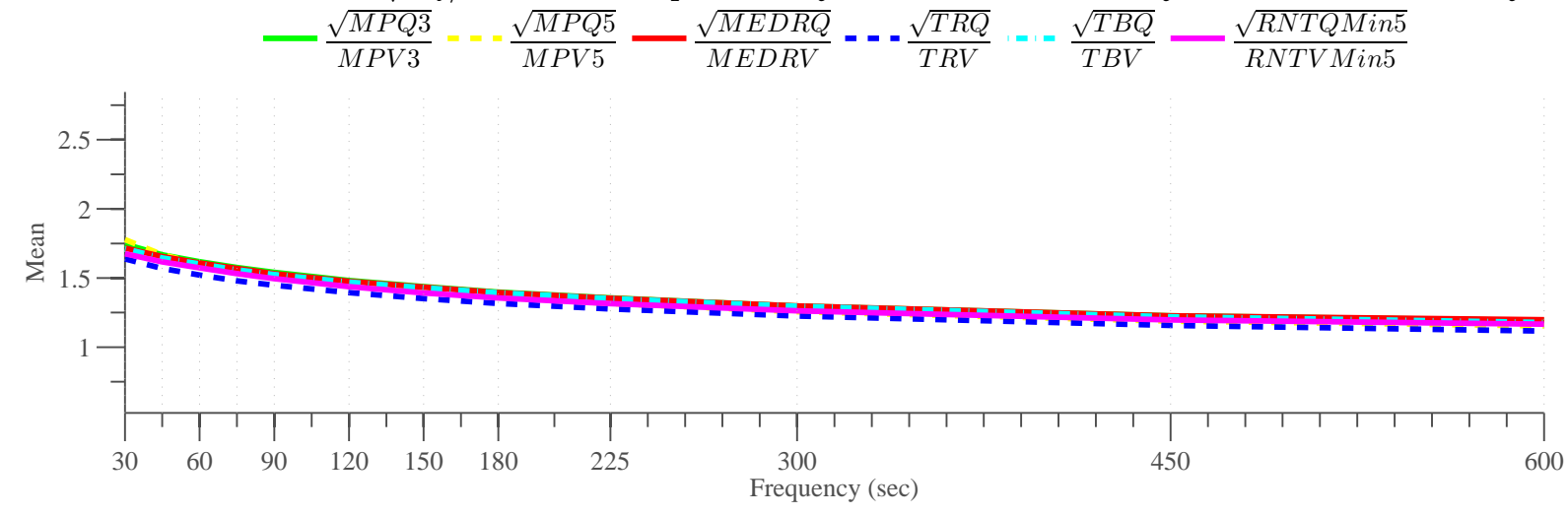

Panel C: Mean of $\sqrt{I Q} / I V$ for the bottom $10 \%$ days in terms of intraday variation of volatility.

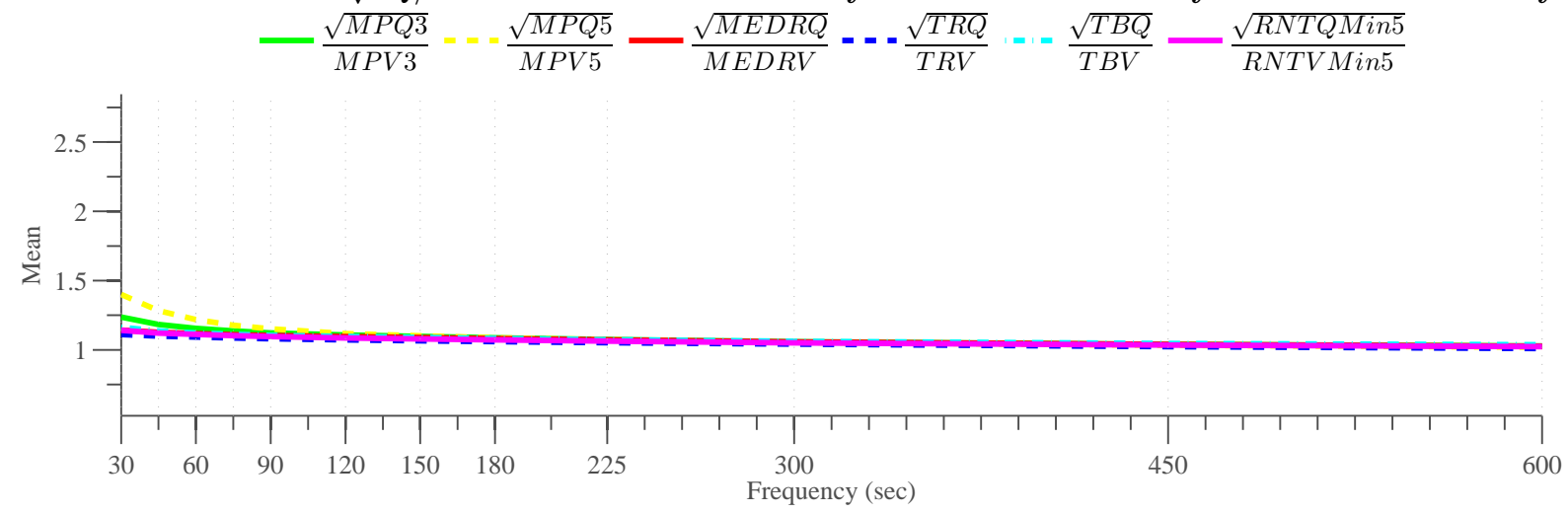


Table 8: Panel data regressions for the period January 1, 2005 - May 31, 2007. We investigate the dependence of the curvature and slope of IV (Panel A), IQ (Panel B) and ratio (Panel C) signature plots on the measure of intraday volatility of volatility $V o V$ described in Section 5. For each estimator $\mathcal{E}$ and frequency $\omega \in\{30 \mathrm{sec}, 60 \mathrm{sec}, \ldots\}$, we run a regression of $\mathcal{E}_{\omega}-\mathcal{E}_{90 \mathrm{sec}}$ (i.e. the estimator compared to itself at a $90 \mathrm{sec}$ base frequency) on $\mathrm{VoV}_{30 \mathrm{sec}}$ and stock fixed effects across a pooled sample of 33 stocks and 606 days between January 2005 and May 2007:

$$
\mathcal{E}_{\omega, i, t}-\mathcal{E}_{90 \mathrm{sec}, i, t}=a_{\omega, i}+b_{\omega} \mathrm{VoV}_{30 \mathrm{sec}, i, t}+u_{\omega, i, t}
$$

In the case of the ratio statistic, the ratio is calculated on each day for each stock. The table reports $\hat{b}_{\omega}$ with robust t-stats in square brackets calculated by two-way clustering by stock and date according to Thompson (2006).

\begin{tabular}{|c|c|c|c|c|c|c|c|}
\hline Estimator Class & $\begin{array}{l}\text { MPV } \\
\text { MPQ }\end{array}$ & $\begin{array}{l}\text { MPV } \\
\text { MPQ }\end{array}$ & $\begin{array}{l}\text { MPV } \\
\text { MPQ }\end{array}$ & $\begin{array}{l}\text { TRV } \\
\text { TRQ }\end{array}$ & $\begin{array}{l}\text { TBV } \\
\text { TBQ }\end{array}$ & $\begin{array}{l}\text { NTV } \\
\text { NTQ }\end{array}$ & $\begin{array}{l}\text { RNTV } \\
\text { RNTQ }\end{array}$ \\
\hline Block Size & 3 & 4 & 5 & 1 & 2 & 3 & 5 \\
\hline Functional & Prod & Prod & Prod & Prod & Prod & Med & Min \\
\hline Selector Index & 1-3 & $1-4$ & $1-5$ & 1 & 1-2 & $1-3$ & $3-5$ \\
\hline \multicolumn{8}{|l|}{ Panel A: $I V$} \\
\hline \multirow{2}{*}{$30 \mathrm{sec}$} & 0.04 & 0.12 & 0.19 & 0.01 & 0.02 & -0.06 & 0.05 \\
\hline & {$[0.43]$} & {$[1.31]$} & {$[1.92]$} & {$[0.10]$} & {$[0.24]$} & {$[-0.66]$} & {$[0.60]$} \\
\hline \multirow{2}{*}{$60 \mathrm{sec}$} & 0.05 & $0.07^{*}$ & $0.09 *$ & $0.07^{*}$ & 0.05 & 0.06 & $0.08^{*}$ \\
\hline & {$[1.64]$} & {$[2.20]$} & {$[2.64]$} & {$[2.76]$} & {$[1.76]$} & {$[1.92]$} & [3.14] \\
\hline \multirow{2}{*}{$120 \mathrm{sec}$} & $-0.07^{*}$ & $-0.07^{*}$ & $-0.08^{*}$ & $-0.08^{*}$ & $-0.06^{*}$ & $-0.07^{*}$ & $-0.10^{*}$ \\
\hline & {$[-4.04]$} & {$[-4.50]$} & {$[-4.47]$} & {$[-5.20]$} & {$[-3.69]$} & {$[-3.84]$} & {$[-6.68]$} \\
\hline \multirow{2}{*}{$180 \mathrm{sec}$} & $-0.21^{*}$ & $-0.22^{*}$ & $-0.25^{*}$ & $-0.21^{*}$ & $-0.20^{*}$ & $-0.23^{*}$ & $-0.27^{*}$ \\
\hline & {$[-5.40]$} & {$[-5.30]$} & {$[-5.62]$} & {$[-5.28]$} & {$[-4.39]$} & {$[-5.20]$} & {$[-6.52]$} \\
\hline \multirow{2}{*}{$300 \mathrm{sec}$} & $-0.44^{*}$ & $-0.50^{*}$ & $-0.54^{*}$ & $-0.49^{*}$ & $-0.45^{*}$ & $-0.46^{*}$ & $-0.51^{*}$ \\
\hline & {$[-6.41]$} & {$[-7.25]$} & {$[-7.65]$} & {$[-7.62]$} & {$[-6.24]$} & {$[-6.92]$} & {$[-7.66]$} \\
\hline \multirow{2}{*}{$600 \mathrm{sec}$} & $-0.71^{*}$ & $-0.76^{*}$ & $-0.82^{*}$ & $-0.79^{*}$ & $-0.83^{*}$ & $-0.74^{*}$ & $-0.77^{*}$ \\
\hline & {$[-7.07]$} & {$[-7.93]$} & {$[-7.79]$} & {$[-8.33]$} & {$[-7.88]$} & {$[-6.72]$} & {$[-7.54]$} \\
\hline \multicolumn{8}{|l|}{ Panel B: $\sqrt{I Q}$} \\
\hline \multirow{2}{*}{$30 \mathrm{sec}$} & $0.31^{*}$ & 0.30 & $0.34^{*}$ & $0.76^{*}$ & $0.52^{*}$ & $0.53^{*}$ & $0.54^{*}$ \\
\hline & {$[2.00]$} & {$[1.92]$} & {$[2.65]$} & {$[6.07]$} & {$[3.79]$} & {$[3.70]$} & {$[4.53]$} \\
\hline \multirow{2}{*}{$60 \mathrm{sec}$} & $0.30 *$ & $0.23^{*}$ & $0.25^{*}$ & $0.41^{*}$ & $0.34^{*}$ & $0.38^{*}$ & $0.33^{*}$ \\
\hline & [5.19] & {$[4.59]$} & {$[5.17]$} & {$[9.40]$} & {$[7.15]$} & {$[6.90]$} & {$[8.40]$} \\
\hline \multirow{2}{*}{$120 \mathrm{sec}$} & $-0.30^{*}$ & $-0.28^{*}$ & $-0.30^{*}$ & $-0.33^{*}$ & $-0.28^{*}$ & $-0.28^{*}$ & $-0.32^{*}$ \\
\hline & {$[-7.67]$} & {$[-8.50]$} & {$[-8.30]$} & {$[-10.37]$} & {$[-10.17]$} & {$[-8.99]$} & {$[-10.18]$} \\
\hline \multirow{2}{*}{$180 \mathrm{sec}$} & $-0.76^{*}$ & $-0.78^{*}$ & $-0.83^{*}$ & $-0.86^{*}$ & $-0.80^{*}$ & $-0.83^{*}$ & $-0.91^{*}$ \\
\hline & {$[-10.55]$} & {$[-9.98]$} & {$[-11.28]$} & {$[-12.01]$} & {$[-10.26]$} & {$[-9.67]$} & {$[-9.84]$} \\
\hline \multirow{2}{*}{$300 \mathrm{sec}$} & $-1.53^{*}$ & $-1.55^{*}$ & $-1.59 *$ & $-1.60^{*}$ & $-1.50^{*}$ & $-1.53^{*}$ & $-1.58^{*}$ \\
\hline & {$[-10.86]$} & {$[-11.38]$} & {$[-11.66]$} & {$[-9.75]$} & {$[-10.11]$} & {$[-10.62]$} & {$[-11.32]$} \\
\hline \multirow{2}{*}{$600 \mathrm{sec}$} & $-2.25^{*}$ & $-2.30^{*}$ & $-2.31^{*}$ & $-2.40^{*}$ & $-2.47^{*}$ & $-2.24^{*}$ & $-2.30 *$ \\
\hline & {$[-9.18]$} & {$[-10.60]$} & {$[-10.90]$} & {$[-10.23]$} & {$[-9.98]$} & {$[-9.31]$} & {$[-9.75]$} \\
\hline \multicolumn{8}{|l|}{ Panel C: $\sqrt{I Q} / I V$} \\
\hline \multirow{2}{*}{$30 \mathrm{sec}$} & $0.32 *$ & 0.09 & -0.17 & $0.68^{*}$ & $0.56^{*}$ & $0.62^{*}$ & $0.55^{*}$ \\
\hline & {$[4.45]$} & {$[0.89]$} & {$[-1.10]$} & {$[17.97]$} & {$[12.26]$} & {$[12.45]$} & [13.12] \\
\hline \multirow{2}{*}{$60 \mathrm{sec}$} & $0.22^{*}$ & $0.19^{*}$ & $0.16^{*}$ & $0.27^{*}$ & $0.27^{*}$ & $0.25^{*}$ & $0.25^{*}$ \\
\hline & {$[8.84]$} & [6.68] & [4.09] & {$[18.67]$} & [14.43] & {$[11.91]$} & [16.07] \\
\hline \multirow{2}{*}{$120 \mathrm{sec}$} & $-0.18^{*}$ & $-0.18^{*}$ & $-0.18^{*}$ & $-0.19^{*}$ & $-0.19 *$ & $-0.18^{*}$ & $-0.20 *$ \\
\hline & {$[-12.43]$} & {$[-11.62]$} & {$[-9.16]$} & {$[-18.10]$} & {$[-17.26]$} & {$[-13.54]$} & {$[-15.18]$} \\
\hline \multirow{2}{*}{$180 \mathrm{sec}$} & $-0.44^{*}$ & $-0.45^{*}$ & $-0.46^{*}$ & $-0.44^{*}$ & $-0.45^{*}$ & $-0.43^{*}$ & $-0.48^{*}$ \\
\hline & {$[-17.13]$} & {$[-15.50]$} & {$[-13.38]$} & {$[-22.31]$} & {$[-19.59]$} & {$[-16.99]$} & {$[-20.13]$} \\
\hline \multirow{2}{*}{$300 \mathrm{sec}$} & $-0.75^{*}$ & $-0.78^{*}$ & $-0.77^{*}$ & $-0.71^{*}$ & $-0.75^{*}$ & $-0.72^{*}$ & $-0.78^{*}$ \\
\hline & {$[-18.08]$} & {$[-17.72]$} & {$[-14.81]$} & {$[-22.77]$} & {$[-21.16]$} & {$[-18.73]$} & {$[-20.96]$} \\
\hline \multirow{2}{*}{$600 \mathrm{sec}$} & $-0.98^{*}$ & $-1.03^{*}$ & $-1.02^{*}$ & $-0.99^{*}$ & $-1.08^{*}$ & $-0.95^{*}$ & $-1.02^{*}$ \\
\hline & {$[-20.31]$} & {$[-20.52]$} & {$[-17.74]$} & {$[-26.21]$} & {$[-23.57]$} & {$[-20.78]$} & {$[-22.35]$} \\
\hline
\end{tabular}


Table 9: Panel data regressions for the period June 1, 2007 - July 31, 2009. We investigate the dependence of the curvature and slope of IV (Panel A), IQ (Panel B) and ratio (Panel C) signature plots on the measure of intraday volatility of volatility $V o V$ described in Section 5. For each estimator $\mathcal{E}$ and frequency $\omega \in\{30 \mathrm{sec}, 60 \mathrm{sec}, \ldots\}$, we run a regression of $\mathcal{E}_{\omega}-\mathcal{E}_{90 \mathrm{sec}}$ (i.e. the estimator compared to itself at a 90sec base frequency) on $\mathrm{VoV}_{30 \mathrm{sec}}$ and stock fixed effects across a pooled sample of 33 stocks and 545 days between June 2007 and July 2009:

$$
\mathcal{E}_{\omega, i, t}-\mathcal{E}_{90 \mathrm{sec}, i, t}=a_{\omega, i}+b_{\omega} \mathrm{VoV}_{30 \mathrm{sec}, i, t}+u_{\omega, i, t}
$$

In the case of the ratio statistic, the ratio is calculated on each day for each stock. The table reports $\hat{b}_{\omega}$ with robust t-stats in square brackets calculated by two-way clustering by stock and date according to Thompson (2006).

\begin{tabular}{|c|c|c|c|c|c|c|c|}
\hline Estimator Class & $\begin{array}{l}\text { MPV } \\
\text { MPQ }\end{array}$ & $\begin{array}{l}\text { MPV } \\
\text { MPQ }\end{array}$ & $\begin{array}{l}\text { MPV } \\
\text { MPQ }\end{array}$ & $\begin{array}{l}\text { TRV } \\
\text { TRQ }\end{array}$ & $\begin{array}{l}\text { TBV } \\
\text { TBQ }\end{array}$ & $\begin{array}{l}\text { NTV } \\
\text { NTQ }\end{array}$ & $\begin{array}{l}\text { RNTV } \\
\text { RNTQ }\end{array}$ \\
\hline Block Size & 3 & 4 & 5 & 1 & 2 & 3 & 5 \\
\hline Functional & Prod & Prod & Prod & Prod & Prod & Med & Min \\
\hline Selector Index & $1-3$ & $1-4$ & $1-5$ & 1 & 1-2 & $1-3$ & $3-5$ \\
\hline \multicolumn{8}{|l|}{ Panel A: $I V$} \\
\hline \multirow{2}{*}{$30 \mathrm{sec}$} & 0.27 & $0.77^{*}$ & $1.12^{*}$ & -0.54 & -0.37 & -0.50 & -0.16 \\
\hline & {$[1.31]$} & {$[2.36]$} & {$[2.30]$} & {$[-0.84]$} & {$[-0.69]$} & {$[-0.75]$} & {$[-0.30]$} \\
\hline \multirow{2}{*}{$60 \mathrm{sec}$} & 0.13 & $0.25^{*}$ & $0.39^{*}$ & -0.09 & -0.01 & -0.12 & 0.09 \\
\hline & {$[1.37]$} & {$[2.27]$} & {$[2.27]$} & {$[-0.41]$} & {$[-0.07]$} & {$[-0.41]$} & {$[0.32]$} \\
\hline \multirow{2}{*}{$120 \mathrm{sec}$} & -0.15 & $-0.24^{*}$ & $-0.35^{*}$ & -0.03 & -0.06 & 0.05 & -0.08 \\
\hline & {$[-1.08]$} & {$[-2.47]$} & {$[-3.45]$} & {$[-0.16]$} & {$[-0.37]$} & {$[0.19]$} & {$[-0.43]$} \\
\hline \multirow{2}{*}{$180 \mathrm{sec}$} & $-0.48^{*}$ & $-0.75^{*}$ & $-0.95^{*}$ & -0.13 & -0.25 & -0.12 & -0.52 \\
\hline & {$[-2.03]$} & {$[-3.50]$} & {$[-3.93]$} & {$[-0.33]$} & {$[-0.76]$} & {$[-0.25]$} & {$[-1.48]$} \\
\hline \multirow{2}{*}{$300 \mathrm{sec}$} & $-1.13^{*}$ & $-1.43^{*}$ & $-1.69 *$ & -0.80 & -0.74 & -0.65 & $-1.06^{*}$ \\
\hline & {$[-3.10]$} & {$[-4.53]$} & {$[-4.36]$} & {$[-1.45]$} & {$[-1.56]$} & {$[-1.17]$} & {$[-2.23]$} \\
\hline \multirow{2}{*}{$600 \mathrm{sec}$} & $-1.65^{*}$ & $-2.04^{*}$ & $-2.36^{*}$ & $-1.66^{*}$ & $-1.67^{*}$ & -1.41 & $-1.74^{*}$ \\
\hline & {$[-3.11]$} & {$[-4.23]$} & {$[-4.27]$} & {$[-2.15]$} & {$[-2.21]$} & {$[-1.69]$} & {$[-2.46]$} \\
\hline \multicolumn{8}{|l|}{ Panel B: $\sqrt{I Q}$} \\
\hline \multirow{2}{*}{$30 \mathrm{sec}$} & 0.85 & 1.20 & $1.95^{*}$ & 1.04 & 0.54 & 0.63 & 1.64 \\
\hline & {$[1.00]$} & {$[1.75]$} & {$[3.05]$} & {$[0.84]$} & {$[0.49]$} & {$[0.55]$} & {$[1.51]$} \\
\hline \multirow{2}{*}{$60 \mathrm{sec}$} & 0.56 & $0.66^{*}$ & $0.86^{*}$ & 0.64 & 0.59 & 0.46 & 0.77 \\
\hline & {$[1.34]$} & {$[2.10]$} & {$[3.21]$} & {$[1.46]$} & {$[1.15]$} & {$[0.78]$} & [1.38] \\
\hline \multirow{2}{*}{$120 \mathrm{sec}$} & $-0.80^{*}$ & $-0.83^{*}$ & $-0.97^{*}$ & $-0.80^{*}$ & $-0.87^{*}$ & -0.63 & $-0.78^{*}$ \\
\hline & {$[-2.38]$} & {$[-2.19]$} & {$[-3.37]$} & {$[-2.75]$} & {$[-2.45]$} & {$[-1.50]$} & {$[-2.75]$} \\
\hline \multirow{2}{*}{$180 \mathrm{sec}$} & $-2.59^{*}$ & $-2.56^{*}$ & $-2.65^{*}$ & $-2.27^{*}$ & $-2.15^{*}$ & $-1.82^{*}$ & $-2.39 *$ \\
\hline & {$[-3.10]$} & {$[-3.62]$} & {$[-4.36]$} & {$[-3.48]$} & {$[-3.37]$} & {$[-2.48]$} & {$[-3.59]$} \\
\hline \multirow{2}{*}{$300 \mathrm{sec}$} & $-4.61^{*}$ & $-4.83^{*}$ & $-5.00 *$ & $-4.67^{*}$ & $-4.27^{*}$ & $-4.26^{*}$ & $-4.64^{*}$ \\
\hline & {$[-3.54]$} & {$[-3.95]$} & {$[-4.72]$} & {$[-4.08]$} & {$[-3.68]$} & {$[-3.60]$} & {$[-4.09]$} \\
\hline \multirow{2}{*}{$600 \mathrm{sec}$} & $-6.47^{*}$ & $-6.88^{*}$ & $-7.12^{*}$ & $-6.92^{*}$ & $-7.12^{*}$ & $-6.71^{*}$ & $-6.61^{*}$ \\
\hline & {$[-3.67]$} & {$[-4.10]$} & {$[-4.35]$} & {$[-4.15]$} & {$[-4.03]$} & {$[-3.62]$} & {$[-4.04]$} \\
\hline \multicolumn{8}{|l|}{ Panel C: $\sqrt{I Q} / I V$} \\
\hline \multirow{2}{*}{$30 \mathrm{sec}$} & $0.38^{*}$ & $0.36^{*}$ & 0.32 & $0.52^{*}$ & $0.46^{*}$ & $0.49^{*}$ & $0.45^{*}$ \\
\hline & {$[5.15]$} & {$[2.96]$} & {$[1.73]$} & {$[13.44]$} & {$[9.79]$} & {$[10.85]$} & {$[12.22]$} \\
\hline \multirow{2}{*}{$60 \mathrm{sec}$} & $0.18^{*}$ & $0.17^{*}$ & $0.17^{*}$ & $0.21^{*}$ & $0.21^{*}$ & $0.21^{*}$ & $0.22^{*}$ \\
\hline & {$[5.81]$} & {$[3.76]$} & {$[3.00]$} & [13.64] & [10.60] & [13.09] & [12.19] \\
\hline \multirow{2}{*}{$120 \mathrm{sec}$} & $-0.15^{*}$ & $-0.16^{*}$ & $-0.17^{*}$ & $-0.16^{*}$ & $-0.15^{*}$ & $-0.16^{*}$ & $-0.16^{*}$ \\
\hline & {$[-8.21]$} & {$[-6.17]$} & {$[-5.21]$} & {$[-15.93]$} & {$[-13.38]$} & {$[-14.24]$} & {$[-18.47]$} \\
\hline \multirow{2}{*}{$180 \mathrm{sec}$} & $-0.39^{*}$ & $-0.40^{*}$ & $-0.40^{*}$ & $-0.40^{*}$ & $-0.39 *$ & $-0.40^{*}$ & $-0.41^{*}$ \\
\hline & {$[-12.96]$} & {$[-9.04]$} & {$[-6.52]$} & {$[-20.39]$} & {$[-19.25]$} & {$[-17.28]$} & {$[-25.00]$} \\
\hline \multirow{2}{*}{$300 \mathrm{sec}$} & $-0.66^{*}$ & $-0.68^{*}$ & $-0.68^{*}$ & $-0.66^{*}$ & $-0.66^{*}$ & $-0.65^{*}$ & $-0.68^{*}$ \\
\hline & {$[-12.49]$} & {$[-9.63]$} & {$[-7.44]$} & {$[-25.11]$} & {$[-22.01]$} & {$[-22.20]$} & {$[-25.25]$} \\
\hline \multirow{2}{*}{$600 \mathrm{sec}$} & $-0.91^{*}$ & $-0.93^{*}$ & $-0.93^{*}$ & $-0.94^{*}$ & $-1.00^{*}$ & $-0.90^{*}$ & $-0.92^{*}$ \\
\hline & {$[-12.82]$} & {$[-9.76]$} & {$[-8.42]$} & {$[-26.28]$} & {$[-21.40]$} & {$[-24.03]$} & {$[-25.59]$} \\
\hline
\end{tabular}




\section{Conclusion}

We provide a first in-depth look at robust estimation of integrated quarticity (IQ) based on high frequency data. We review the basic theory and provide a novel set of jump-robust estimators that are defined in terms of order statistics of suitable return functionals and generalize the existing nearest neighbor truncation estimators of ADS (2010). This new class of robust neighborhood truncation (RNT) estimators can be designed to enhance the robustness properties vis-à-vis microstructure noise features of the data as well as reducing the finite sample sensitivity to outliers. We find that such RNT estimators outperform existing estimators by a considerable margin in terms of efficiency in estimating the key ratio of IQ over IV squared. The latter quantity is extremely useful for robust inference regarding IV and in testing for price jumps, as documented by BNS (2002).

In the empirical implementation, we emphasize the importance of appropriate filtering for gross violations of the particular null hypothesis associated with a given estimation procedure. In particular, we develop a novel functional filtering scheme for local power variation estimators, which generalizes truncation of individual returns to truncation of return functionals and is easy to apply for a broad range of popular estimators. By invoking this new approach to filtering directly at the level of the local power variation estimators, the threshold can be set very conservatively, thereby avoiding systematic biases arising from overly aggressive truncation and eliminating the need for ex-post bias correction.

The unifying theme behind the proposed universal filtering procedure and new class of estimators is to operate directly on the functional space of local power variation estimates instead of restricting attention to the underlying individual returns, which can be viewed as a special case. Combining the proposed novel filtering procedure with the new RNT class of estimators enables efficient inference in an extensive simulation design and delivers sensible results in an empirical application to the set of Dow Jones 30 stocks. Overall, the study provides a set of new guidelines for the construction of practical robust and efficient estimation and inference regarding IV and IQ. 


\section{References}

Andersen, T. G. and T. Bollerslev. Answering the skeptics: Yes, standard volatility models do provide accurate forecasts. International Economic Review 39.

Andersen, T. G., T. Bollerslev, and F. X. Diebold (2007). Roughing it up: Including jump components in the measurement, modeling, and forecasting of return volatility. Review of Economics and Statistics 89.

Andersen, T. G., T. Bollerslev, and F. X. Diebold (2010). Parametric and Nonparametric Volatility Measurement. North Holland. In Handbook of Financial Econometrics, Volume 1 - Tools and Techniques; Yacine Aït-Sahalia, Lars P. Hansen, and Jose A. Scheinkman (Eds.).

Andersen, T. G., T. Bollerslev, F. X. Diebold, and P. Labys (2001). The distribution of realized exchange rate volatility. Journal of the American Statistical Association 96, 42-55.

Andersen, T. G., T. Bollerslev, F. X. Diebold, and P. Labys (2003). Modeling and forecasting realized volatility. Econometrica 71.

Andersen, T. G., T. Bollerslev, and D. Dobrev. No-arbitrage semi-martingale restrictions for continuous-time volatility models subject to leverage effects, jumps and i.i.d. noise: Theory and testable distributional implications. Journal of Econometrics 138.

Andersen, T. G., D. P. Dobrev, and E. Schaumburg (2008). Duration based volatility estimation. Manuscript, Northwestern University.

Andersen, T. G., D. P. Dobrev, and E. Schaumburg (2009). Jump-robust volatility estimation using nearest neighbor truncation. NBER Working Paper (15533).

Back, K. Asset pricing for general processes. Journal of Mathematical Economics 20.

Bandi, F. M. and J. R. Russell (2008). Microstructure noise, realized variance, and optimal sampling. Review of Economic Studies 75.

Barndorff-Nielsen, O. E., S. E. Graversen, J. Jacod, M. Podolskij, and N. Shephard (2006). A central limit theorem for realized power and bipower variations of continuous semimartingales. in From stochastic calculus to mathematical finance : the Shiryaev Festschrift, Springer Verlag.

Barndorff-Nielsen, O. E., S. E. Graversen, J. Jacod, and N. Shephard. Limit theorems for bipower variation in financial econometrics. Econometric Theory 22.

Barndorff-Nielsen, O. E., P. R. Hansen, A. Lunde, and N. Shephard (2008). Designing realized kernels to measure the ex post variation of equity prices in the presence of noise. Econometrica $76,1481-1536$.

Barndorff-Nielsen, O. E., P. R. Hansen, A. Lunde, and N. Shephard (2009). Realised kernels in practice: Trades and quotes. Econometrics Journal 12, C1-C32.

Barndorff-Nielsen, O. E. and N. Shephard. Econometric analysis of realized covariation: High frequency based covariance, regression, and correlation in financial economics. Econometrica 72.

Barndorff-Nielsen, O. E. and N. Shephard. Econometrics of testing for jumps in financial economics using bipower variation. Journal of Financial Econometrics 4.

Barndorff-Nielsen, O. E. and N. Shephard. Estimating quadratic variation using realized variance. Journal of Applied Econometrics 17. 
Barndorff-Nielsen, O. E. and N. Shephard. Power and bipower variation with stochastic volatility and jumps. Journal of Financial Econometrics 2.

Barndorff-Nielsen, O. E., N. Shephard, and M. Winkel (2006). Limit theorems for multipower variation in the presence of jumps. Stochastic Processes and Their Applications 116, 796-806.

Christensen, K., R. Oomen, and M. Podolskij (2008). Realized quantile-based estimation of integrated variance. Working paper; CREATES, University of Aarhus, Denmark.

Christensen, K., R. Oomen, and M. Podolskij (2010). Realized quantile-based estimation of integrated variance. Journal of Econometrics 159, 74-98.

Corsi, F., D. Pirino, and R. Renò (2010). Threshold bipower variation and the impact of jumps on volatility forecasting. Journal of Econometrics 159.

Hasbrouck, J. The dynamics of discrete bid and ask quotes. Journal of Finance 54 .

Hsieh, D. A. Chaos and nonlinear dynamics: Applications to financial markets. Journal of Finance 46.

Huang, X. and G. Tauchen. The relative contribution of jumps to total price variance. Journal of Financial Econometrics 3.

Jacod, J., Y. Li, P. Mykland, M. Podolskij, and M. Vetter (2009). Mirostructure noise in the continuous case: the pre-averaging approach. Stochastic Processes and Their Applications 26.

Jacod, J. and P. Protter (1998). Asymptotic error distributions for the euler method for stochastic differential equations. Annals of Probability 26.

Jacod, J. and A. N. Shiryaev (2003). Limit theorems for stochastic processes (2nd ed.). Grundlehren der mathematischen Wissenschaften. Berlin; New York: Springer.

Jiang, G. J. and R. C. Oomen (2008). Testing for jumps when asset prices are observed with noise - a "swap variance" approach. Journal of Econometrics 144.

Mancini, C. (2009). Non-parametric threshold estimation for models with stochastic diffusion coefficient and jumps. Scandinavian Journal of Statistics 36.

Mykland, P. A. and L. Zhang (2009). Inference for continuous semimartingales observed at high frequency. Econometrica $7 \%$.

Podolskij, M. and M. Vetter (2009). Estimation of volatility functionals in the simultaneous presence of microstructure noise and jumps. Bernoulli 15, 634-658.

Schwert, G. W. Why does stock market volatility change over time? The Journal of Finance 44.

Zhang, L., P. A. Mykland, and Y. Aït-Sahalia. A tale of two time scales: Determining integrated volatility with noisy high-frequency data. Journal of the American Statistical Association 100.

Zhou, B. (1996). High-frequency data and volatility in foreign-exchange rates. Journal of Business and Economic Statistics 14. 


\section{A Proofs of Propositions}

This appendix provides proofs of Propositions 1-4 for the general case of estimating the integrated power variation of order $p$, where $p$ is a positive and even integer. The proofs are initially given for the MinPV and MedPV type estimators and subsequently shown to extend to the RNT estimators in Lemma 12 below.

The (integrated) power variation, $P V(p)$, is formally defined as,

$$
P V(p)=\int_{0}^{1} \sigma_{u}^{p} d u
$$

Obviously, $p=4$ corresponds to the theoretical quantity relevant for the $M i n R Q$ and $M e d R Q$ estimators while $p=2$ refers to the integrated variance underlying the $\operatorname{Min} R V$ and $M e d R V$ estimators. The higher order integrated power variation estimators are less commonly used but do appear in the recent literature. For example, $p=8$ is required to assess the (asymptotic) precision of integrated quarticity estimators.

\section{A.1 Basic Setting}

Let $Y_{t}$ be the $\log$ price process following a Brownian semimartingale

$$
Y_{t}=Y_{0}+\int_{0}^{t} a_{u} d u+\int_{0}^{t} \sigma_{u_{-}} d B_{u}
$$

where $a$ is a locally bounded and predictable process and $\sigma$ is adapted, cadlag and bounded away from zero. Without loss of generality, we further assume that the functions $a, \sigma$ are uniformly bounded and $\inf _{t>0} \sigma_{t}>0$ a.s. ${ }^{27}$ The extension allowing for finite activity jumps in $Y_{t}$ is dealt with Section A.6 below.

When discussing central limit theorems (CLTs) we require in addition that the volatility process follows a generalized Itô process:

$$
\text { Assumption (A1) : } \sigma_{t}=\sigma_{0}+\int_{0}^{t} \tilde{a}_{u} d u+\int_{0}^{t} \tilde{\sigma}_{u_{-}} d B_{u}+\int_{0}^{t} \tilde{v}_{u_{-}} d W_{u}
$$

where $\tilde{a}$ is locally bounded and predictable and $\tilde{\sigma}, \tilde{v}$ are cadlag and the Brownian motions $B, W$ are uncorrelated. We impose, without loss of generality, that the functions $\tilde{a}$, $\tilde{\sigma}$, and $\tilde{v}$ are uniformly bounded as well

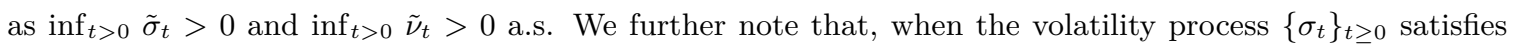
Assumption A1, then the power variation process, $\left\{\sigma_{t}^{2}\right\}_{t \geq 0}$, also conforms to this general characterization.

We assume $Y$ is observed at $N+1$ evenly spaced time points spanning the interval $[0 ; 1]$. Below, we denote these observations by $Y_{i / N}, i=0, \ldots, N$, and the associated log-returns by $\Delta_{i}^{N} Y=Y_{i / N}-Y_{(i-1) / N}, i=$ $1, \ldots, N$. The proofs involve sequences of standardized return observations and corresponding approximating sequences for which volatility is fixed across one or more returns. Hence, we introduce non-overlapping blocks of $M \geq 1$ returns for which the volatility process is constant. We assume we have $K=N / M$ such blocks in the sample. Consequently, we define the quantities,

$$
\begin{aligned}
\chi_{i}^{N} & =\sqrt{N} \Delta_{i}^{N} Y, \\
\beta_{i}^{N, M} & =\sqrt{N} \sigma_{\frac{\lfloor(i-1) / M\rfloor M}{N}} \Delta_{i}^{N} B=\sqrt{N} \sigma_{\frac{\lfloor(i-1) / M\rfloor}{K}} \Delta_{i}^{N} B,
\end{aligned}
$$

where $\lfloor\cdot\rfloor$ indicates the integer part of an expression. Hence, for each of the $K$ return blocks, corresponding to $\beta_{i}^{N, M}$, the volatility remains fixed at the value it attains at the beginning of the block.

\footnotetext{
${ }^{27}$ As argued in Barndorf-Nielsen, Graversen, Podolskij, Jacod and Shephard (2006), henceforth BNGJPS, this follows from working with the stopped versions of the processes: $T_{t}^{(k)}=Y_{t \wedge T_{k}}$ and $\sigma_{t}^{(k)}=\sigma_{t \wedge T_{k}}$ where $T_{k}=\inf \left\{t|| a_{t}|+| \sigma_{t_{-}} \mid \geq k\right\}$ and $T_{k} \nearrow \infty$ a.s.
} 


\section{A.2 The Min and Med Power Variation Estimators}

Let $p$ be a fixed positive even integer and let $g_{m i n, p}: \mathbb{R}^{2} \mapsto \mathbb{R}_{+}$be given by,

$$
\begin{aligned}
g_{\text {min,p }}(\boldsymbol{a}) & =d_{\min , p} \min \left(\left|a_{1}\right|^{p},\left|a_{2}\right|^{p}\right), \quad \text { where the scaling constant takes the form, } \\
d_{\min , p} & =\left(E\left[\min \left(\left|Z_{1}\right|^{p},\left|Z_{2}\right|^{p}\right)\right]\right)^{-1}, \quad \text { and } \quad Z_{1}, Z_{2} \sim \text { i.i.d.N } \mathcal{N}(0,1)
\end{aligned}
$$

For example, we have $d_{\min , 2}=\frac{\pi}{\pi-2} \quad$ and $\quad d_{\min , 4}=\frac{\pi}{3 \pi-8}$.

Similarly, we define the median-based function $g_{m e d, p}: \mathbb{R}^{3} \mapsto \mathbb{R}_{+}$and scaling factors,

$$
\begin{aligned}
g_{\text {med,p }, p}(\boldsymbol{a}) & =d_{\text {med,p }} \operatorname{med}\left(\left|a_{1}\right|^{p},\left|a_{2}\right|^{p},\left|a_{3}\right|^{p}\right), \text { where } \\
d_{\text {med,p }} & \left.=\left(E\left[\operatorname{med}\left(\left|Z_{1}\right|^{p},\left|Z_{2}\right|^{p}\right),\left|Z_{3}\right|^{p}\right)\right]\right)^{-1}, \quad \text { and } \quad Z_{1}, Z_{2}, Z_{3} \sim \text { i.i.d.N }(0,1) .
\end{aligned}
$$

In this case, $d_{m e d, 2}=\frac{\pi}{6-4 \sqrt{3}+\pi}$ and $\quad d_{m e d, 4}=\frac{3 \pi}{9 \pi+72-52 \sqrt{3}}$.

For any even positive integer, $p$, we define the nearest neighbor truncation estimators of the $p^{\prime}$ th order power variation by,

$$
\begin{aligned}
& \operatorname{MinPV}_{N}(p)=d_{m i n, p} \frac{1}{N-1} \sum_{i=1}^{N-1} \min \left(\left(\chi_{i}^{N}\right)^{p},\left(\chi_{i+1}^{N}\right)^{p}\right)=\frac{1}{N-1} \sum_{i=1}^{N-1} g_{m i n, p}\left(\chi_{i}^{N}, \chi_{i+1}^{N}\right), \\
& \operatorname{MedPV}_{N}(p)=d_{m e d, p} \frac{1}{N-2} \sum_{i=1}^{N-2} \min \left(\left(\chi_{i}^{N}\right)^{p},\left(\chi_{i+1}^{N}\right)^{p},\left(\chi_{i+2}^{N}\right)^{p}\right)=\frac{1}{N-2} \sum_{i=1}^{N-2} g_{m e d, p}\left(\chi_{i}^{N}, \chi_{i+1}^{N}, \chi_{i+2}^{N}\right) .
\end{aligned}
$$

For the cases of primary interest, i.e., $p=2$ and $p=4$, these estimators are identical to the Min and Med estimators introduced in Section 2.1. Specifically, we have

$$
\begin{array}{ll}
\operatorname{MinPV}_{N}(2)=\operatorname{MinRV}_{N}, & \operatorname{MedPV}_{N}(2)=\operatorname{MedRV}_{N}, \\
\operatorname{MinPV}_{N}(4)=\operatorname{MinRQ}_{N}, & \operatorname{MedPV}_{N}(2)=\operatorname{MedRQ}_{N} .
\end{array}
$$

\section{A.3 Additional Notation and Preliminary Results}

We provide a detailed proof of the results in Propositions 1 and 2 concerning the $\operatorname{MinPV}_{N}(p)$ estimator. The proofs for $\operatorname{MedPV}_{N}(p)$ may be derived similarly. Moreover, we henceforth consider a fixed even, positive integer, $p$, so the $g_{\min , p}$ function is uniquely defined. We refer to it simply as $g$ below.

First, we observe that, for any bivariate vectors, $\boldsymbol{a}=\left(a_{1}, a_{2}\right)$ and $\boldsymbol{b}=\left(b_{1}, b_{2}\right)$, we have the following useful bound,

$$
|g(\boldsymbol{a})-g(\boldsymbol{b})| \leq d_{\min , p}\left(\left|a_{1}^{p}-b_{1}^{p}\right|+\left|a_{2}^{p}-b_{2}^{p}\right|\right),
$$

and furthermore that, except on the null set $\left\{\left(a_{1}, a_{2}\right) \in \mathbb{R}^{2} \mid a_{1}=a_{2}\right\}$, we have

$$
\lim _{\varepsilon \rightarrow 0} \frac{1}{\varepsilon}\left[\min \left(a_{1}^{p}, a_{2}^{p}+\varepsilon z\right)-\min \left(a_{1}^{p}, a_{2}^{p}\right)\right]=\left\{\begin{array}{lll}
z & \text { if } & \left|a_{2}\right|<\left|a_{1}\right| \\
0 & \text { if } & \left|a_{2}\right|>\left|a_{1}\right|
\end{array}\right.
$$

The proofs of Propositions 1 and 2 revolve around the sequences,

$$
\boldsymbol{V}_{N}=\frac{1}{N} \sum_{i=1}^{N-1} g\left(\chi_{i}^{N}, \chi_{i+1}^{N}\right) \quad \text { and } \quad \boldsymbol{U}_{N}^{M}=\frac{1}{N} \sum_{i=1}^{N-1} g\left(\beta_{i}^{N, M}, \beta_{i+1}^{N, M}\right) .
$$


Since $\operatorname{MinPV}_{N}(p)=\frac{N}{N-1} V_{N}$, the $V_{N}$ sequence is asymptotically equivalent to our $\operatorname{MinPV}$ estimator, while $U_{N}^{M}$ is an approximating sequence as, for large $N, \chi_{i}^{N} \approx \beta_{i}^{N, M}$.

For any adapted, integrable, $d$-dimensional cadlag process, $X$, and for $N \geq j>i-1 \geq 0$, we define the expectation conditional on information at time $\frac{i-1}{N}$ :

$$
E_{i-1}\left[X_{\frac{j}{N}}\right]=E\left[X_{\frac{j}{N}} \mid \mathcal{F}_{\frac{i-1}{N}}\right]
$$

A useful implication of our ability to focus on the case with uniformly bounded drift and volatility functions is that, using the Burkholder-Davis-Grundy inequalities, we have,

$$
E_{i-1}\left[\left|\chi_{i}^{N}\right|^{q}\right] \leq C \text { and } E_{i-1}\left[\left|\beta_{i}^{N, M}\right|^{q}\right] \leq C
$$

where $q>0$ and $C$ denotes a generic positive constant which we (with slight abuse of notation) allow to take on disparate values in different places.

We decompose our estimators for the power variation, $P V(p)$, into a sum of conditional expectations and the associated martingale difference sequences: $V_{N}=V_{1 N}+V_{2 N}$ and $U_{N}^{M}=U_{1 N}^{M}+U_{2 N}^{M}$ where,

$$
\begin{gathered}
\boldsymbol{V}_{1 N}=\frac{1}{N} \sum_{i=1}^{N-1} E_{i-1}\left[g\left(\chi_{i}^{N}, \chi_{i+1}^{N}\right)\right], \quad \boldsymbol{V}_{2 N}=\frac{1}{N} \sum_{i=1}^{N-1}\left\{g\left(\chi_{i}^{N}, \chi_{i+1}^{N}\right)-E_{i-1}\left[g\left(\chi_{i}^{N}, \chi_{i+1}^{N}\right)\right]\right\} \\
\boldsymbol{U}_{1 N}^{M}=\frac{1}{N} \sum_{i=1}^{N-1} E_{\left\lfloor\frac{i-1}{M}\right\rfloor M}\left[g\left(\beta_{i}^{N, M}, \beta_{i+1}^{N, M}\right)\right], \quad \boldsymbol{U}_{2 N}^{M}=\frac{1}{N} \sum_{i=1}^{N-1}\left\{g\left(\beta_{i}^{N, M}, \beta_{i+1}^{N, M}\right)-E_{\left\lfloor\frac{i-1}{M}\right\rfloor M}\left[g\left(\beta_{i}^{N, M}, \beta_{i+1}^{N, M}\right)\right]\right\} .
\end{gathered}
$$

When $M=1$ we will use the shorthand $\beta_{i}^{N} \equiv \beta_{i}^{N, 1}, \boldsymbol{U}_{N} \equiv \boldsymbol{U}_{N}^{1}$ and similarly for the individual pieces $\boldsymbol{U}_{1 N}$ and $\boldsymbol{U}_{2 N}$. These definitions allow us to decompose the main estimator:

$$
\boldsymbol{V}_{N}=\boldsymbol{U}_{1 N}+\boldsymbol{U}_{2 N}+\left(\boldsymbol{V}_{1 N}-\boldsymbol{U}_{1 N}\right)+\left(\boldsymbol{V}_{2 N}-\boldsymbol{U}_{2 N}\right)
$$

Consistency of $V_{N}$ can then be obtained by showing consistency of the estimator applied to the approximating Brownian path with piecewise constant volatility $\left(\boldsymbol{U}_{N}=\boldsymbol{U}_{1 N}+\boldsymbol{U}_{2 N}\right)$ and then showing that the difference $V_{N}-U_{N}$ (the last two terms in (12) above) is asymptotically negligible. This is what we do in Section A.4 below. To prove a CLT, we exploit a different decomposition (similar to Mykland and Zhang (2007)), in which we show the CLT for our estimator applied to an approximating Brownian motion for which volatility is piecewise constant over blocks of length $M$. We then proceed to show that the difference between the original estimator and the estimator applied to the approximating process is negligible. This analysis is carried out in Section A.5 based on the decomposition:

$$
\begin{aligned}
\sqrt{N}\left(\boldsymbol{V}_{N}-P V(p)\right) & =\sqrt{N}\left(\boldsymbol{V}_{1 N}-\boldsymbol{U}_{1 N}\right)+\sqrt{N}\left(\boldsymbol{V}_{2 N}-\boldsymbol{U}_{2 N}\right)+\sqrt{N}\left(\boldsymbol{U}_{1 N}-P V(p)\right) \\
& +\sqrt{N}\left(\boldsymbol{U}_{2 N}-\boldsymbol{U}_{2 N}^{M}\right)+\sqrt{N} \boldsymbol{U}_{2 N}^{M}
\end{aligned}
$$

\section{A.4 Proposition 2: Consistency}

We proceed by analyzing equation (12) term by term through a series of lemmas. For brevity, we focus on the features that are specific to our estimator, while referring to proofs in the extant literature when feasible. This also serves to highlight the underlying structural similarities between our $P V(p)$ measure and previously proposed power variation estimators and, in particular, $I V$ and $I Q$ estimators. 
Lemma 5 Under the maintained assumptions we have,

$$
\boldsymbol{U}_{1 N} \stackrel{P}{\rightarrow} P V(p)
$$

Moreover, if Assumption (A1) holds we obtain,

$$
\sqrt{N}\left(U_{1 N}-P V(p)\right) \stackrel{P}{\rightarrow} 0
$$

Proof. First, note that

$$
g\left(\beta_{i}^{N}, \beta_{i+1}^{N}\right)=\left[g\left(\beta_{i}^{N}, \beta_{i+1}^{N}\right)-g\left(\beta_{i}^{N}, \sqrt{N} \sigma_{\frac{i-1}{N}} \Delta_{i+1}^{N} B\right)\right]+g\left(\beta_{i}^{N}, \sqrt{N} \sigma_{\frac{i-1}{N}} \Delta_{i+1}^{N} B\right)
$$

so we may write

$$
\boldsymbol{U}_{1 N}=\frac{1}{N} \sum_{i=1}^{N-1} \mathbb{E}_{i-1}\left[g\left(\beta_{i}^{N}, \beta_{i+1}^{N}\right)-g\left(\beta_{i}^{N}, \sqrt{N} \sigma_{\frac{i-1}{N}} \Delta_{i+1}^{N} B\right)\right]+\frac{1}{N} \sum_{i=1}^{N-1} \sigma_{\frac{i-1}{N}}^{p}
$$

The first sum in (16) tends to zero in probability. To see this, note that the bound (8) implies the following limit in $L_{2}$-norm:

$$
\mathbb{E}\left|\frac{1}{N} \sum_{i=1}^{N-1} \mathbb{E}_{i-1}\left[g\left(\beta_{i}^{N}, \beta_{i+1}^{N}\right)-g\left(\beta_{i}^{N}, \sqrt{N} \sigma_{\frac{i-1}{N}} \Delta_{i+1}^{N} B\right)\right]\right|^{2} \leq \frac{C}{N} \mathbb{E}\left[\sum_{i=1}^{N-1}\left|\sigma_{\frac{i}{N}}^{p}-\sigma_{\frac{i-1}{N}}^{p}\right|^{2}\right] \rightarrow 0
$$

where the convergence (17), and thus also convergence in probability, follows from the fact that $\sigma_{t}^{p}$ has finite quadratic variation (since $\sigma_{t}$ is a cadlag semimartingale). In addition, since $\left\{\sigma_{t}^{p}\right\}_{t \geq 0}$ is uniformly bounded and cadlag, the pointwise dominated convergence of $\left(\sigma_{u}^{p}-\sigma_{\frac{\lfloor u N\rfloor}{N}}^{p}\right) \rightarrow 0$ for $u \in[0 ; 1]$ follows and Lebesgue's theorem yields

$$
\sum_{i=1}^{N-1}\left[\int_{\frac{(i-1)}{N}}^{\frac{i}{N}}\left(\sigma_{u}^{p}-\sigma_{\frac{(i-1)}{N}}^{p}\right) d u\right] \stackrel{a . s .}{\rightarrow} 0
$$

Together (17) and (18) imply $P V(p)-U_{1 N} \rightarrow 0$, which establishes (14). To show (15) we need the stronger assumption (A1). Define the sequence of independent standard normals $Z_{i}=\sqrt{N} \Delta_{i}^{N} B$, then Assumption (A1) yields

$$
\mathbb{E}_{i-1}\left[\left(\sigma_{\frac{i}{N}}^{p}-\sigma_{\frac{i-1}{N}}^{p}\right) Z_{i+1}^{p} \mathbf{1}_{\left|Z_{i+1}\right|<\left|Z_{i}\right|}\right]=\mathbb{E}_{i-1}\left[\left(\sigma_{\frac{i}{N}}^{p}-\sigma_{\frac{i-1}{N}}^{p}\right) \varphi\left(Z_{i}\right)\right]=O_{P}(1 / N)
$$

since $\varphi\left(Z_{i}\right)=\mathbb{E}_{i}\left[Z_{i+1}^{p} \mathbf{1}_{\left|Z_{i+1}\right|<\left|Z_{i}\right|}\right]$ is an even function of the Brownian path $\left\{B_{t}\right\}_{(i-1) / N<t<i / N}$. Now the property (9) yields

$$
\mathbb{E}_{i-1}\left[g\left(\beta_{i}^{N}, \beta_{i+1}^{N}\right)-g\left(\beta_{i}^{N}, \sqrt{N} \sigma_{\frac{i-1}{N}} \Delta_{i+1}^{N} B\right)\right]=O_{P}(1 / N)
$$

This ensures that the first term in (16) is asymptotically negligible, even when scaled up by $\sqrt{N}$. Hence, the remaining task is to show,

$$
\sqrt{N}\left(\frac{1}{N} \sum_{i=1}^{N-1} \sigma_{\frac{i-1}{N}}^{p}-P V(p)\right) \stackrel{P}{\rightarrow} 0
$$


However, this is analogous to the common task of showing that

$$
\sqrt{N}\left(\frac{1}{N} \sum_{i=1}^{N-1} \sigma_{\frac{i-1}{N}}^{2}-I V\right) \stackrel{P}{\rightarrow} 0
$$

in the IV literature and the method of proof is, by now, well established; see, e.g., BNGJPS where the result is shown in a general setting (allowing for infinite activity jumps) of which the current framework is a special case. A more intuitive and detailed exposition is provided by Barndorff-Nielsen, Graversen, Jacod, and Shephard (2006), henceforth BNGJS.

Lemma 6 Under the maintained assumptions, we have

$$
\boldsymbol{U}_{2 N} \stackrel{P}{\rightarrow} 0
$$

Proof. To simplify notation, define the martingale difference sequence $\left\{\frac{1}{N} \eta_{i}^{N}, \mathcal{F}_{\frac{i}{N}}\right\}_{i \geq 0}$ :

$$
\eta_{i}^{N}=g\left(\beta_{i}^{N}, \beta_{i+1}^{N}\right)-E_{i-1}\left[g\left(\beta_{i}^{N}, \beta_{i+1}^{N}\right)\right]
$$

Note that $\mathbb{E}\left[\left(\eta_{i}^{N}\right)^{2} \mid \mathcal{F}_{\frac{i-1}{N}}\right] \leq C$, so applying the Cauchy-Schwartz inequality,

$\boldsymbol{V}\left[\frac{1}{N} \sum_{i=1}^{N} \eta_{i}^{N}\right]=\frac{1}{N} \mathbb{E}\left[\frac{1}{N} \sum_{i=1}^{N}\left(\left(\eta_{i}^{N}\right)^{2}+2 \eta_{i}^{N} \eta_{i+1}^{N}\right)\right] \leq \frac{C}{N} \mathbb{E}\left[\frac{1}{N} \sum_{i=1}^{N} \mathbb{E}\left[\left(\eta_{i}^{N}\right)^{2} \mid \mathcal{F}_{\frac{i-1}{N}}\right]\right] \leq \frac{C}{N} \rightarrow 0$

The $L_{2}$ convergence implies $\frac{1}{N} \sum_{i=1}^{N} \eta_{i}^{N} \stackrel{P}{\rightarrow} 0$.

Lemma 7 Under the maintained assumptions, we have,

$$
\left(\boldsymbol{V}_{1 N}-\boldsymbol{U}_{1 N}\right) \stackrel{P}{\rightarrow} 0
$$

Under Assumption (A1), we obtain,

$$
\sqrt{N}\left(\boldsymbol{V}_{1 N}-\boldsymbol{U}_{1 N}\right) \stackrel{P}{\rightarrow} 0 .
$$

Proof. We must show,

$$
V_{1 N}-U_{1 N}=\frac{1}{N} \sum_{i=1}^{N-1} \mathbb{E}_{i-1}\left[\left(g\left(\chi_{i}^{N}, \chi_{i+1}^{N}\right)-g\left(\beta_{i}^{N}, \beta_{i+1}^{N}\right)\right)\right] \rightarrow 0 \text { as } N \rightarrow \infty .
$$

Using the bound (8), it follows that,

$$
\begin{aligned}
V_{1 N}-U_{1 N} & \leq \frac{1}{N} \mathbb{E}\left[\sum_{i=1}^{N-1}\left|g\left(\chi_{i}^{N}, \chi_{i+1}^{N}\right)-g\left(\beta_{i}^{N}, \beta_{i+1}^{N}\right)\right|\right] \\
& \leq \frac{C}{N} \mathbb{E}\left[\sum_{i=1}^{N}\left|\left(\chi_{i}^{N}\right)^{p}-\left(\beta_{i}^{N}\right)^{p}\right|\right]=\frac{C}{N} \sum_{i=1}^{N}\left(\mathbb{E}_{i-1}\left|h\left(\sqrt{N} \Delta_{i}^{N} Y\right)-\sigma_{\frac{i-1}{N}}^{p}\right|\right)
\end{aligned}
$$

where we have defined the function $h(x)=x^{p}$. This formulation maps directly into the setting of BNGJPS where the results of this lemma are proven in a more general setting and for a generic $h(x)$ function subject to regularity conditions. In particular, our $h$ function trivially satisfies the continuous differentiability and polynomial growth conditions necessary for the applicability of their analysis. An accessible, albeit lengthy, 
account of the steps of the argument may be found in BNGJS (2006, pp. 713-719). So while this proof is quite involved, the above reformulation of the relevant inequalities, as they arise within our specific setting, allows us to simply refer to previously published work for the result.

Lemma 8 Under the maintained assumptions, we have,

$$
\left(\boldsymbol{V}_{2 N}-\boldsymbol{U}_{2 N}\right) \stackrel{P}{\rightarrow} 0
$$

Moreover, we may strengthen this result further to obtain,

$$
\sqrt{N}\left(\boldsymbol{V}_{2 N}-\boldsymbol{U}_{2 N}\right) \stackrel{P}{\rightarrow} 0 .
$$

Proof. In order to demonstrate the second result of the lemma, which obviously implies the first, we define,

$$
\xi_{i}^{N}=(1 / \sqrt{N})\left[g\left(\chi_{i}^{N}, \chi_{i+1}^{N}\right)-g\left(\beta_{i}^{N}, \beta_{i+1}^{N}\right)\right]
$$

and we must then prove that,

$$
\sum_{i=1}^{N-1}\left(\xi_{i}^{N}-\mathbb{E}_{i-1}\left[\xi_{i}^{N}\right]\right) \stackrel{P}{\rightarrow} 0
$$

This is a martingale difference sequence with respect to the filtration $\mathcal{F}_{\frac{i}{N}}$, so it suffices to show,

$$
\sum_{i=1}^{N-1} \mathbb{E}\left[\left(\xi_{i}^{N}\right)^{2}\right]=\mathbb{E}\left[\sum_{i=1}^{N-1} \mathbb{E}_{i-1}\left[\left(\xi_{i}^{N}\right)^{2}\right]\right] \rightarrow 0 \quad \text { as } \quad N \rightarrow \infty
$$

Mimicking the type of steps undertaken in the proof of the previous lemma, including application of the uniform bound on moments of $\chi_{i}^{N}$ and $\beta_{i}^{N}$, we obtain,

$$
\begin{aligned}
\sum_{i=1}^{N-1} \mathbb{E}\left[\left(\xi_{i}^{N}\right)^{2}\right] & =\frac{1}{N} \mathbb{E}\left[\sum_{i=1}^{N-1} \mathbb{E}_{i-1}\left|g\left(\chi_{i}^{N}, \chi_{i+1}^{N}\right)-g\left(\beta_{i}^{N}, \beta_{i+1}^{N}\right)\right|^{2}\right] \\
& \leq \frac{C}{N} \mathbb{E}\left[\sum_{i=1}^{N} \mathbb{E}_{i-1}\left[\left(h\left(\chi_{i}^{N}\right)-h\left(\beta_{i}^{N}\right)\right)^{2}\right]\right]
\end{aligned}
$$

As for the previous lemma, our reformulation of the task maps the problem into the corresponding task in BNGJPS (2006) who prove a corresponding lemma in a more general setting. A detailed account of the requisite steps to complete this part of the proof may again be gleaned from BNGJS (2006, pp. 704-706).

Taken together, Lemma $5-6$ and the first parts of Lemma $7-8$ imply the consistency of our estimator under the minimal maintained assumptions. The second parts of Lemmas $7-8$ are critical for the proof of the central limit theorem below.

\section{A.5 Proposition 3: The CLT}

Lemma 9 Under assumption (A1), we have

$$
\sqrt{N} \boldsymbol{U}_{2 N}^{M} \stackrel{\text { stable }}{\longrightarrow} \mathcal{D} \mathcal{N}\left(0, \nu \int_{0}^{1} \sigma^{2 p} d u\right)
$$

where the constant $\nu=\operatorname{Var}\left[g\left(Z_{0}, Z_{1}\right]+2 \mathbb{C o v}\left[g\left(Z_{0}, Z_{1}\right), g\left(Z_{1}, Z_{2}\right)\right]\right.$ for $Z_{0}, Z_{1}, Z_{2} \sim$ i.i.d.N $(0,1)$. 
Proof. Consider splitting the $N$ scaled return observations into $K$ blocks, the $k^{\text {th }}$ of which is the vector $\chi_{k}^{M}=\left\{\sqrt{N} \Delta_{i}^{N} Y\right\}_{i \in\{(k-1) M+1, \ldots, k M\}}$. The corresponding vector of observations from the approximating Brownian motion where volatility is held constant over the block is $\boldsymbol{\beta}_{k}^{N, M}=\left\{\beta_{i}^{N, M}\right\}_{i \in\{(k-1) M+1, \ldots, k M\}}$. Next, define by $g_{M}(\cdot): \mathbb{R}^{M} \mapsto \mathbb{R}$ the block estimator of volatility:

$$
g_{M}\left(\boldsymbol{\beta}_{k}^{N, M}\right)=\frac{1}{M} \sum_{i=(k-1) M+1}^{k M-1} g\left(\beta_{i}^{N, M}, \beta_{i+1}^{N, M}\right)
$$

We wish to apply Theorem IX.7.28 in Jacod and Shiryaev (2003) to $\sqrt{N} \boldsymbol{U}_{2 N}^{M}$. Defining the martingale difference sequence $\psi_{k}^{N, M}=\sqrt{M}\left(g_{M}\left(\boldsymbol{\beta}_{k}^{N, M}\right)-\frac{M-1}{M} \sigma_{\frac{(k-1)}{K}}^{2}\right)$ we can write

$$
\begin{aligned}
\sqrt{N} \boldsymbol{U}_{2 N}^{M} & =\frac{1}{\sqrt{K}} \sum_{k=1}^{K} \psi_{k}^{N, M}+\frac{1}{\sqrt{N}} \sum_{k=1}^{K-1}\left(g\left(\beta_{k M}^{N, M}, \beta_{k M+1}^{N, M}\right)-\mathbb{E}_{\frac{k-1}{K}}\left[g\left(\beta_{k M}^{N, M}, \beta_{k M+1}^{N, M}\right)\right]\right) \\
& =\frac{1}{\sqrt{K}} \sum_{k=1}^{K} \psi_{k}^{N, M}+o_{P}(1)
\end{aligned}
$$

The last equality follows from the fact that each term in the second sum is centered and has bounded variance (given the uniform bound on $\sigma_{t}$ ). Thus the sum divided by $\sqrt{N}$ will tend to zero provided $K=o_{P}(N)$ ).

We must now verify conditions (7.27)-(7.31) of Theorem IX.7.28. First note that $E\left[\psi_{k}^{N, M} \mid \mathcal{F}_{\frac{k-1}{K}}\right]=0$ so that condition (7.27) is trivially satisfied. Condition (7.28) follows from the fact that

$$
\frac{1}{K} \sum_{k=1}^{K} E\left[\left\{\sqrt{M}\left(g_{M}\left(\boldsymbol{\beta}_{k}^{M}\right)-\frac{M-1}{M} \sigma_{\frac{(k-1)}{K}}^{p}\right)\right\}^{2} \mid \mathcal{F}_{\frac{k-1}{K}}\right]=\frac{\nu}{K} \sum_{k=1}^{K} \sigma_{\frac{k-1}{K}}^{2 p} \stackrel{P}{\rightarrow} \nu \int_{0}^{1} \sigma_{u}^{2 p} d u
$$

where the convergence in probability (and in fact a.s.) is a consequence of the volatility process being cadlag and uniformly bounded. Next, we turn to condition $(7.29)$. Let $\Delta_{k}^{M} \boldsymbol{B}=\left(B_{\frac{k}{K}}-B_{\frac{(k-1)}{K}}\right)$, then $E\left[\psi_{k}^{N, M} \Delta_{k}^{M} \boldsymbol{B} \mid \mathcal{F}_{\frac{k-1}{K}}\right]=0$, which follows from the fact that the variables $\psi_{k}^{N, M}$ are centered and that $g_{M}$ is an even function. Condition (7.30), stating that $E\left[\left(\psi_{k}^{N, M}\right)^{2} \mathbf{1}_{\left|\psi_{k}^{N, M}\right|>\varepsilon}\right] \stackrel{P}{\rightarrow} 0$, follows straightforwardly from the fact that $\sigma$ is uniformly bounded.

Finally, let $\left\{N_{t}\right\}_{t \in[0 ; 1]}$ be a bounded martingale orthogonal to $B$ (i.e. the covariation $\langle B, N\rangle_{t}=0$ a.s.). We want to show that, for each block $k, E\left[\psi_{k}^{N, M}\left(N_{\frac{k}{K}}-N_{\frac{(k-1)}{K}}\right) \mid \mathcal{F}_{\frac{k-1}{K}}\right]=0$. For $t>\frac{k-1}{K}$ consider the martingale difference sequence $M_{t}=E\left[\psi_{k}^{N, M} \mid \mathcal{F}_{t}\right]$. By the martingale representation theorem, $M_{t}=$ $M_{\frac{k-1}{K}}+\int_{\frac{k-1}{K}}^{\frac{k}{K}} \varphi_{u} d B_{u}$ for some predictable process $\varphi_{u}$. Therefore the processes $\left\{M_{t}\right\}_{t>\frac{k-1}{K}}$ and $\left\{N_{t}-\right.$ $\left.N_{\frac{k-1}{K}}\right\}_{t>\frac{k-1}{K}}$ are orthogonal and the product, $\left\{M_{t}\left(N_{t}-N_{\frac{k-1}{K}}\right)\right\}$ is again a martingale which must then have mean zero. This verifies condition (7.31) and Theorem IX.7.28 in Jacod and Shiryaev (2003) states that as $N$ (and hence $K$ and $M$ ) tend to infinity:

$$
\sqrt{N} \boldsymbol{U}_{2 N}^{M} \stackrel{\text { stable }}{\longrightarrow} \mathcal{N}\left(0, \nu \int_{0}^{1} \sigma^{2 p} d u\right)
$$

Lemma 10 Under the maintained assumptions, we have

$$
\sqrt{N}\left(\boldsymbol{U}_{2 N}-\boldsymbol{U}_{2 N}^{M}\right) \stackrel{P}{\rightarrow} 0
$$


Proof. Defining $\eta_{i}^{N, M}=g\left(\beta_{i}^{N, M}, \beta_{i+1}^{N, M}\right)-\mathbb{E}_{\lfloor(i-1) / M\rfloor M}\left[g\left(\beta_{i}^{N, M}, \beta_{i+1}^{N, M}\right)\right]$, we note that $\left\{\frac{1}{\sqrt{N}}\left(\eta_{i}^{N}-\eta_{i}^{N, M}\right)\right\}_{i \geq 1}$ is a martingale difference sequence with respect to the filtration $\left\{\mathcal{F}_{i / N}\right\}$. To show that $\sqrt{N}\left(\boldsymbol{U}_{2 N}-\boldsymbol{U}_{2 N}^{M}\right)=$ $\sum_{i=1}^{N-1}\left(\eta_{i}^{N}-\eta_{i}^{N, M}\right) / \sqrt{N} \rightarrow 0$ in probability, it therefore suffices (by Doobs inequality, e.g. Revuz and Yor (1999)) to show that

$$
\frac{1}{N} \mathbb{E}\left[\sum_{i=1}^{N-1}\left|g\left(\beta_{i}^{N}, \beta_{i+1}^{N}\right)-g\left(\beta_{i}^{N, M}, \beta_{i+1}^{N, M}\right)\right|^{2}\right] \rightarrow 0
$$

By the bound of $g(\cdot)$ we have

$$
\begin{aligned}
& \frac{1}{N} \mathbb{E}\left[\sum_{i=1}^{N-1}\left|g\left(\beta_{i}^{N}, \beta_{i+1}^{N}\right)-g\left(\beta_{i}^{N, M}, \beta_{i+1}^{N, M}\right)\right|^{2}\right] \leq \frac{C}{N} \mathbb{E}\left[\sum_{i=1}^{N} \mathbb{E}_{i-1}\left|\left(\beta_{i}^{N}\right)^{p}-\left(\beta_{i}^{N, M}\right)^{p}\right|^{2}\right] \\
& \leq \frac{C}{N} \mathbb{E}\left[\sum_{i=1}^{N}\left|\sigma_{\frac{i-1}{N}}^{p}-\sigma_{\frac{\lfloor(i-1) / M\rfloor M}{N}}^{p}\right|^{2}\right]=C \mathbb{E} \int_{0}^{1}\left(\sigma_{\frac{\lfloor u N\rfloor}{N}}^{p}-\sigma_{\frac{\lfloor u K\rfloor}{K}}^{p}\right)^{2} d u=o_{P}(1),
\end{aligned}
$$

where the last inequality follows from the uniform boundedness of $\sigma_{t}$ and Lebesgues theorem.

Importantly, the specification of the volatility process in Assumption (A1) may be extended to include finite as well as infinite activity jump processes subject only to the regularity conditions stipulated in BNGJPS. This follows from the fact that the only terms in (13) affected by the inclusion of jumps are the terms $\sqrt{N}\left(V_{1 N}-U_{1 N}\right)$ and $\sqrt{N}\left(V_{2 N}-U_{2 N}\right)$ which map into the corresponding terms in BNGJPS as outlined in the proofs above. As such, the distributional results of the paper cover a wide range of underlying return generating processes.

\section{A.6 The Asymptotic Distribution under Jump Alternatives}

Suppose now the log price process is given as $X=Y+J$, where $Y$ is a Brownian semimartingale of the form (4) while $J$ is a finite activity jump process. We show below that the above results continue to hold ${ }^{28}$ The key is that $\sqrt{N}\left|Y_{\frac{i}{N}}-Y_{\frac{i-1}{N}}\right|=O_{P}\left(|\log (N)|^{1 / 2}\right)$, which follows readily from Levy's modulus of continuity theorem for Brownian motion. This immediately yields:

Proposition 11 When $J$ is a finite activity jump process, the asymptotic distribution of the minPV $(p)$ and $\operatorname{MedPV}(p)$ estimators applied to the processes $\left\{X_{t}\right\}$ and $\left\{Y_{t}\right\}$ are identical.

Proof. As before, we deal only with the $\operatorname{MinPV}$ case as the $M e d P V$ case is analogous. On a given realization of the path there is a finite number of jumps, so (asymptotically) at most one of the terms $\left|X_{\frac{i}{N}}-X_{\frac{i-1}{N}}\right|$ or $\left|X_{\frac{i+1}{N}}-X_{\frac{i}{N}}\right|$ includes a jump. Therefore, each term in the estimator (up to a normalizing constant) is

$$
\min \left(\left|X_{\frac{i}{N}}-X_{\frac{i-1}{N}}\right|^{p},\left|X_{\frac{i+1}{N}}-X_{\frac{i}{N}}\right|^{p}\right)=O_{P}\left(\frac{(\log N)^{p / 2}}{N}\right)
$$

\footnotetext{
${ }^{28}$ As for the volatility process, the specification may be generalized to infinite activity jump processes along the lines of Barndorff-Nielsen et al (2006c).
} 
regardless of whether a (single) jump occurred or not over $\left[\frac{i-1}{N}, \frac{i+1}{N}\right]$. Since only finitely many terms differ,

$$
\begin{array}{r}
\sum_{j=1}^{N}\left[\min \left(\left|X_{\frac{i}{N}}-X_{\frac{i-1}{N}}\right|^{p},\left|X_{\frac{i+1}{N}}-X_{\frac{i}{N}}\right|^{p}\right)-\min \left(\left|Y_{\frac{i}{N}}-Y_{\frac{i-1}{N}}\right|^{p},\left|Y_{\frac{i+1}{N}}-Y_{\frac{i}{N}}\right|^{p}\right)\right] \\
=O_{P}\left(\frac{(\log N)^{p / 2}}{N}\right)=o_{P}\left(\frac{1}{\sqrt{N}}\right)
\end{array}
$$

so neither consistency nor convergence in distribution is affected by the presence of finite activity jumps.

\section{A.7 Robust Neighborhood Truncation Estimators}

We consider the family of robust neighborhood truncation (RNT) estimators on a block of $m$ i.i.d. $\mathcal{N}\left(0, \sigma^{2}\right)$ returns, $\left\{Z_{1}, \ldots, Z_{m}\right\}$. The estimator is then constructed by taking the $j^{\text {th }}$ quantile of $H$ unbiased estimators of $\sigma^{p}$ on the block. Denoting these primitive estimators by $\mathcal{E}_{1}, \ldots, \mathcal{E}_{H}$, we can write the RNT estimator as

$$
R N T_{N}^{(j, I)}(p)=d_{(j, I)}(p) \frac{1}{(N-m+1)} \sum_{i=1}^{N-m+1} q_{j}\left[\mathcal{E}_{1}, \ldots, \mathcal{E}_{H}\right]
$$

where the $d_{(j, I)}(p)$ is a scaling factor.

Lemma 12 Let $p$ be a positive even integer and assume that the estimators $\mathcal{E}_{1}, \ldots, \mathcal{E}_{H}$ satisfy the conditions of Proposition 1 and Proposition 2, then the robust neighborhood truncation estimator, $R N T_{N}^{(j, \mathbf{I})}(p)$, defined in (35) is consistent for $\sigma^{p}$ and satisfies a CLT.

Proof. We need to verify the three properties,(8)-(9) and symmetry, of the $g(\dot{)}$ function used in the theorem are satisfied when $g(\cdot)=R N T_{N}^{(j, I)}(p)$. We deal with each condition in turn.

Clearly, if each primitive estimator $\mathcal{E}_{i}$ is symmetric, so is $R N T_{N}^{(j, I)}(p)$. Moreover, if each $\mathcal{E}_{i}$ satisfies a bound of the type (8), so will $R N T_{N}^{(j, I)}(p)$ as it is simply an order statistic of such bounded functionals. Finally, assume that each $\mathcal{E}_{i}$ satisfies (9). Except on a null set, there exists a neighborhood around each m-tuple $\left(z_{1}, \ldots, z_{m}\right)$, on which $R N T_{N}^{(j, I)}(p)=\mathcal{E}_{i}\left(z_{1}^{p}, \ldots, z_{m}^{p}\right)$ for some $1 \leq i \leq H$. Therefore it follows that also $R N T_{N}^{(j, I)}(p)$ satisfies (9).

Remark 13 Since the NT estimators (up to a scale factor) essentially are a special case of RNT, the lemma applies to these as well.

\section{B Noise Robustness Properties of the Ratio $I Q / I V^{2}$}

The ratio $I Q / I V^{2}$ plays an important role for both IV inference and jump attribution in finite samples. This section extends the analysis of Huang and Tauchen (2005) to show that the ratio $I Q / I V^{2}$ has certain desirable robustness features in the presence of microstructure noise. Following Ait-Sahalia and Mykland (2005), we assume that the true price process $\left(p_{i}^{*}\right)$ is observed at $N+1$ discrete points in time with an independent stationary (possibly autocorrelated) measurement error $\left(u_{i}\right)$ that results in an MA error structure in observed returns:

$$
p_{i}=p_{i}^{*}+u_{i} \Rightarrow r_{i}=r_{i}^{*}+\varepsilon_{i} \text { where } \varepsilon_{i}=u_{i}-u_{i-1}
$$

For simplicity, we focus the discussion here on the $(\mathrm{RV}, \mathrm{RQ})$ pairing and denote:

$$
\widehat{I V}=\sum_{i=1}^{N} r_{i}^{2}, I V^{*}=\sum_{i=1}^{N} r_{i}^{* 2}, \widehat{I Q}=\frac{N}{3} \sum_{i=1}^{N} r_{i}^{4}, I Q^{*}=\frac{N}{3} \sum_{i=1}^{N} r_{i}^{* 4}
$$


The presence of microstructure noise produces a bias in both the IQ and IV estimators of the form:

$$
\begin{aligned}
& E[\widehat{I Q}]= E\left[I Q^{*}\right]+\frac{N^{2}}{3} E\left[\varepsilon_{i}^{4}\right]+2 N E\left[I V^{*}\right] E\left[\varepsilon_{i}^{2}\right] \\
& E\left[\widehat{I V}^{2}\right]=E\left[I V^{* 2}\right]+N E\left[\varepsilon_{i}^{4}\right]+N(N-1)\left(E\left[\varepsilon_{i}^{2}\right]\right)^{2}+2(N+2) E\left[I V^{*}\right] E\left[\varepsilon_{i}^{2}\right] \\
&+2 \sum_{m=1}^{N-1}(N-m) \operatorname{Cov}\left(\varepsilon_{1}^{2}, \varepsilon_{1+m}^{2}\right)
\end{aligned}
$$

In the special case where $u_{i} \sim \mathcal{N}\left(0, \sigma_{u}^{2}\right)$ is serially uncorrelated and denoting the noise to signal ratio $\lambda=\sigma_{u}^{2} /\left(\frac{1}{N} E\left[I V^{*}\right]\right)$, the expressions above simplify to

$$
\begin{aligned}
E[\widehat{I Q}] & =E\left[I Q^{*}\right]+4 E\left[I V^{* 2}\right]\left(\lambda+\lambda^{2}\right) \\
E\left[\widehat{I V}^{2}\right] & =E\left[I V^{* 2}\right]+4 E\left[I V^{* 2}\right]\left(\lambda+\lambda^{2}\right)+O\left(\frac{1}{N}\right)
\end{aligned}
$$

Under the null of no jumps, $I Q^{*}, I V^{*}$ are asymptotically unbiased and consistent and

$$
\frac{\widehat{I Q}}{\widehat{I V}^{2}} \stackrel{P}{\longrightarrow} \frac{I Q+4 I V^{2}\left(\lambda+\lambda^{2}\right)}{I V^{2}+4 I V^{2}\left(\lambda+\lambda^{2}\right)} \text { as } N \rightarrow \infty
$$

The downwards bias of the limiting ratio depends on the noise-to-signal ratio $\lambda$ and preaveraging of returns therefore plays an important role in reducing $\lambda$ and the associated distortions. ${ }^{29}$ Moreover, for sufficiently pre-averaged returns, there is very little evidence of serial correlation, as pointed out by COP (2010), and the serially uncorrelated noise case considered above is therefore the empirically most relevant case. ${ }^{30}$ In finite samples, this downward bias is further compounded by a pure Jensen (concavity) effect as readily seen from the Monte Carlo results for the Brownian motion scenario Path by path, the CauchySchwartz inequality of course implies that $\widehat{I Q}_{/ \widehat{I V}^{2}}^{2} \geq \frac{1}{3}$ must hold regardless of the noise structure or other imperfections.

In the presence of other deviations from the Brownian null, forming the ratio $\widehat{I Q} / \widehat{I V}^{2}$ may have a stabilizing effect provided that the resulting distortion is uniform and roughly proportional to squared returns since this will lead to a cancelation in numerator and denominator. We see this effect at work in the simulations with price discreteness but in other instances, e.g., sparsity, it clearly fails. In cases involving additive distortions such as jumps, there will be no cancelation of biases and the ratio will tend to (in the case of upward biases) diverge at high frequencies due to the scaling by $\mathrm{N}$ in the numerator.

${ }^{29}$ By Hölder's inequality, $I Q \geq I V^{2}$, so that the distortion due to microstructure noise in general will result in a downward bias in the limiting ratio.

${ }^{30}$ In the general case where $\varepsilon_{i}$ is a MA(q) process: $\varepsilon_{i}=\nu_{i}+\sum_{k=1}^{q} \theta_{k} \nu_{i-k}$, we have

$$
\begin{aligned}
E[\widehat{I Q}] & =E\left[I Q^{*}\right]+E\left[I V^{2}\right]\left(\theta_{1}^{2}+\ldots+\theta_{q}^{2}+1\right)^{2} \lambda^{2}+2 E\left[I V^{2}\right]\left(\theta_{1}^{2}+\ldots+\theta_{q}^{2}+1\right) \lambda \\
E\left[\widehat{I V}^{2}\right] & =E\left[I V^{* 2}\right]+E\left[I V^{2}\right]\left(\theta_{1}^{2}+\ldots+\theta_{q}^{2}+1\right)^{2} \lambda^{2}+2 E\left[I V^{2}\right]\left(\theta_{1}^{2}+\ldots+\theta_{q}^{2}+1\right) \lambda+O\left(\frac{1}{N}\right)
\end{aligned}
$$




\section{C $I V$ and $I Q$ estimators based on pre-averaged returns}

Given $N$ equally-spaced $(\log )$ returns, $r_{i}=Y_{\frac{i}{N}}-Y_{\frac{i-1}{N}}, \quad i=1, \cdots, N$, we define corresponding pre-averaged returns for any pre-averaging window size $2 K \leq N$ :

$$
\bar{r}_{i}=\frac{1}{K} \sum_{j=K}^{2 K-1} Y_{\frac{i+j}{N}}-\frac{1}{K} \sum_{j=0}^{K-1} Y_{\frac{i+j}{N}}, i=1, \cdots, N-2 K+1 .
$$

An equivalent definition with analytically more tractable expression is given by:

$$
\bar{r}_{i}=2 \sum_{j=1}^{2 K-1} g\left(\frac{j}{2 K}\right) r_{i+j}, i=1, \cdots, N-2 K+1,
$$

where $g(x)=x \wedge(1-x), x \in[0,1]$ is the pre-averaging kernel. We further define $\psi_{K}=\frac{1}{2 K} \sum_{j=1}^{2 K-1} 4 g\left(\frac{j}{2 K}\right)^{2}$ as the finite sample analog of the variance scaling factor $\psi=\int_{0}^{1} 4 g(u)^{2} d u=\frac{1}{3}$ induced by the pre-averaging kernel. ${ }^{31}$

Consider the following $2 K$ sub-samples of non-overlapping pre-averaged returns:

$$
\begin{aligned}
\bar{S}_{1} & =\left\{\bar{r}_{1+2(s-1) K}: s=1, \ldots,\left\lfloor\frac{N}{2 K}\right\rfloor\right\} \\
\bar{S}_{2} & =\left\{\bar{r}_{2+2(s-1) K}: s=1, \ldots,\left\lfloor\frac{N-1}{2 K}\right\rfloor\right\} \\
& \cdots \\
\bar{S}_{2 K} & =\left\{\bar{r}_{2 K+2(s-1) K}: s=1, \ldots,\left\lfloor\frac{N-2 K+1}{2 K}\right\rfloor\right\}
\end{aligned}
$$

Let $\widehat{I V}\left[\bar{S}_{i}\right]$ and $\widehat{I Q}\left[\bar{S}_{i}\right], i=1, \cdots, 2 K$ denote the raw $I V$ and $I Q$ estimates obtained on each subsample of pre-averaged returns. Then the pre-averaged (and sub-sampled) estimators $\widehat{I V}[\bar{r}$. $]$ and $\widehat{I Q}[\bar{r}$. $]$ for the full set of pre-averaged returns $\bar{r} .=\left\{\bar{r}_{i}\right\}_{i=1}^{N-2 K+1}$ can be defined as follows:

$$
\begin{aligned}
& \widehat{I V}[\bar{r} .]=\frac{1}{2 K} \sum_{i=1}^{2 K} \frac{1}{\psi} \widehat{I V}\left[\bar{S}_{i}\right] \\
& \widehat{I Q}[\bar{r} .]=\frac{1}{2 K} \sum_{i=1}^{2 K} \frac{1}{\psi^{2}} \widehat{I Q}\left[\bar{S}_{i}\right]
\end{aligned}
$$

After incorporating finite sample bias correction, these take the following final form that we use in our pre-averaged implementation of all estimators:

$$
\begin{aligned}
\widehat{I V}^{A d j}\left[\bar{r}_{.}\right] & =\frac{1}{2 K} \sum_{i=1}^{2 K} \frac{1}{\psi_{K}} \frac{\frac{N}{2 K}}{\left\lfloor\frac{N-i+1}{2 K}\right\rfloor} \widehat{I V}\left[\bar{S}_{i}\right] \\
\widehat{I Q}^{A d j}\left[\bar{r}_{.}\right] & =\frac{1}{2 K} \sum_{i=1}^{2 K} \frac{1}{\psi_{K}^{2}} \frac{\left(\frac{N}{2 K}\right)^{2}}{\left\lfloor\frac{N-i+1}{2 K}\right\rfloor^{2}} \widehat{I Q}\left[\bar{S}_{i}\right]
\end{aligned}
$$

Consistency and asymptotic normality are clearly preserved by pre-averaging and sub-sampling, while noise-robustness improves. In particular, bounce-backs are near perfectly annihilated given that adjacent returns are subject to almost identical kernel weights. For further details on pre-averaging please refer to Podolskij and Vetter (2009) and Jacod, Li, Mykland, Podolskij and Vetter (2009) among others.

\footnotetext{
${ }^{31}$ Note that the factor 4 arises from the multiplication by 2 in equation (38).
} 


\section{Tabulating Moments of Order Statistics for Standard Gaus- sian Return Blocks}

Table 10 provides the second and fourth moments of all order statistics for Gaussian return blocks of dimension two through five.

Table 10: We compute the second and fourth moments of order statistics based on blocks of powers of independent standard normals, $Z_{i} \sim \mathcal{N}(0,1)$. Panel A: Expectation of order statistics of squared normals. Panel B: Expectation of order statistics of normals raised to the $4^{\text {th }}$ power.

\begin{tabular}{|c|c|c|c|c|c|}
\hline \multicolumn{6}{|c|}{ Panel A: $2^{\text {nd }}$ moments } \\
\hline Block Size & $Z_{(1)}^{2}$ & $Z_{(2)}^{2}$ & $Z_{(3)}^{2}$ & $Z_{(4)}^{2}$ & $Z_{(5)}^{2}$ \\
\hline 2 & $\begin{array}{c}\frac{\pi-2}{\pi} \\
=1.636619772367581\end{array}$ & $\begin{array}{c}\frac{2+\pi}{\pi} \\
=0.3633802276324187\end{array}$ & & & \\
\hline 3 & $\begin{array}{c}\frac{-6+2 \sqrt{3}+\pi}{\pi} \\
=0.1927984737408401\end{array}$ & $\begin{array}{c}\frac{6-4 \sqrt{3}+\pi}{\pi} \\
=0.7045437354155758\end{array}$ & $\begin{array}{c}1+\frac{2 \sqrt{3}}{\pi} \\
=2.102657790843584\end{array}$ & & \\
\hline 4 & $\begin{array}{c}1+\frac{4(4 \sqrt{3}-9)}{3 \pi} \\
=0.1207021413774029\end{array}$ & $\begin{array}{c}\frac{12-8 \sqrt{3}+\pi}{\pi} \\
=0.4090874708311517\end{array}$ & 1 & $\begin{array}{c}1+\frac{8}{\sqrt{3} \pi} \\
=2.470210387791445\end{array}$ & \\
\hline \multirow[t]{2}{*}{5} & . & . & . & . & . \\
\hline & $=0.08307731289708446$ & $=0.2712014552986765$ & $=0.6159164941298644$ & $=1.256055670580090$ & $=2.773749067094284$ \\
\hline \multicolumn{6}{|c|}{ Panel B: $4^{\text {th }}$ moments } \\
\hline Block Size & $Z_{(1)}^{4}$ & $Z_{(2)}^{4}$ & $Z_{(3)}^{4}$ & $Z_{(4)}^{4}$ & $Z_{(5)}^{4}$ \\
\hline 2 & $\begin{array}{c}3-\frac{8}{\pi} \\
=0.4535209105296746\end{array}$ & $\begin{array}{c}3+\frac{8}{\pi} \\
=5.546479089470325\end{array}$ & & & \\
\hline 3 & $\begin{array}{c}3+\frac{\frac{26}{\sqrt{3}}-24}{\pi} \\
=0.1387464919112216\end{array}$ & $\begin{aligned} & \frac{72-52 \sqrt{3}+9 \pi}{3 \pi} \\
= & 1.083069747766581\end{aligned}$ & $\begin{array}{c}3+\frac{26}{\sqrt{3} \pi} \\
=7.778183760322198\end{array}$ & & \\
\hline 4 & $\begin{array}{c}3+\frac{4(4(13 \sqrt{3}-27) \pi-9)}{9 \pi^{2}} \\
=0.05766408946789071\end{array}$ & $\begin{array}{c}3+\frac{4(9+(36-26 \sqrt{3}) \pi)}{3 \pi^{2}} \\
=0.3819936992412144\end{array}$ & $\begin{array}{c}3-\frac{12}{\pi^{2}} \\
=1.784145796291947\end{array}$ & $\begin{array}{c}3+\frac{4(9+26 \sqrt{3} \pi)}{9 \pi^{2}} \\
=9.776196414998948\end{array}$ & \\
\hline \multirow[t]{2}{*}{5} & $\cdot$ & $\cdot$ & $\cdot$ & $\cdot$ & $\cdot$ \\
\hline & $=0.02855480767257635$ & $=0.1741012166491482$ & $=0.6938324231293139$ & $=2.511021378400369$ & $=11.59249017414859$ \\
\hline
\end{tabular}

